\title{
Quantum dot-sensitized solar cells
}

Zhenxiao Pan, ${ }^{\mathrm{a}}$ Huashang Rao, ${ }^{\mathrm{a}}$ Iván Mora-Seró*b ${ }^{* \mathrm{~b}}$ Juan Bisquert*b and Xinhua Zhong*a

${ }^{a}$ College of Materials and Energy, South China Agricultural University, Guangzhou 510642, China

${ }^{\mathrm{b}}$ Institute of Advanced Materials (INAM), Universitat Jaume I, 12006 Castelló, Spain

*Corresponding authors

Email: sero@uji.es (I. M.)

bisquert@fca.uji.es (J. B.)

zhongxh@scau.edu.cn (X. Z.) 


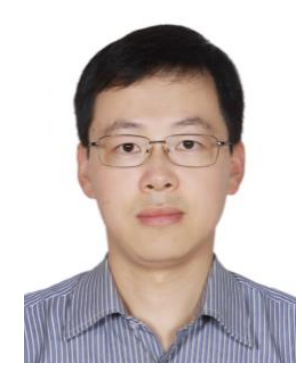

Zhenxiao Pan received his Ph.D. degree in applied chemistry from East China University of Science and Technology in 2015. Currently he is a Professor of South China Agricultural University. His research interests focused on the materials and interface design for high efficiency quantum dot-sensitized solar cells.

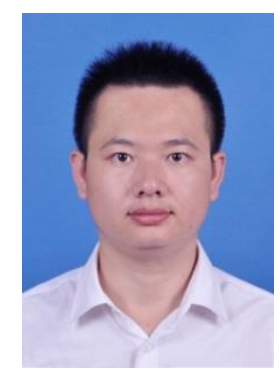

Huashang Rao is an associate professor of South China Agricultural University, China. He received his B.E. and Ph.D. degree at Sun Yat-sen University in 2012 and 2017, respectively. His current research interest lies in the field of new energy materials and their applications in quantum dot/perovskite based solar cells.

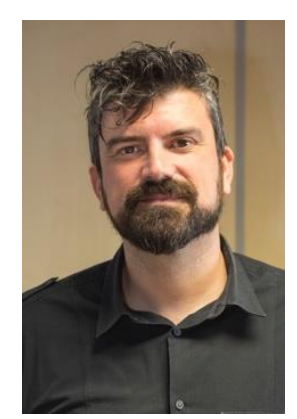

Iván Mora-Seró is associated professor at the Instutut of Advanced Materials (INAM) at Universitat Jaume I de Castelló. His research involves quantum dots and halide perovskites for the development of optoelectronic devices and solar cells. 


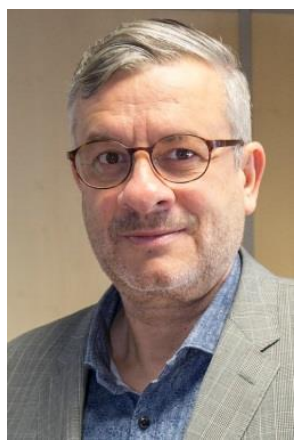

Juan Bisquert is a professor of applied physics at Universitat Jaume I de Castelló and the director of the Institute of Advanced materials (INAM). His main topics of interest are perovskite solar cells and solar fuel production. He has developed the application of measurement techniques and physical modeling that relate the device operation with the elementary steps that take place at the nanoscale dimension.

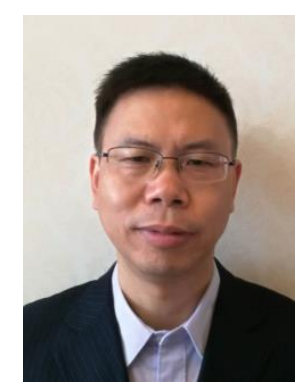

Xinhua Zhong is a professor of South China Agricultural University. Before current position, he worked at East China University of Science and Technology, China. His research mainly involves nanoscaled materials and their applications in energy conversion and storage. His research group has created the efficiency records for quantun dot-sensitized solar cells since 2012. 


\begin{abstract}
Quantum dot-sensitized solar cells (QDSCs) have emerged as a promising candidate for the next-generation solar cells benefitting from distinct optoelectronic features of quantum dot (QD) light-harvesting materials such as high light, thermal, and moisture stability, facilely tunable absorption range, high absorption coefficient, multiple exciton generation possibility, solution processability as well as facile and low-cost availability. In recent years, we have been witnessing a dramatic boost in power conversion efficiency (PCE) of QDSCs from 5\% to nearly $13 \%$, a competitive level with other kinds of emerging solar cells. Both the exploration of new QD light harvesting materials and interface engineering contribute to this fantastically fast improvement. The outstanding development trend of QDSCs indicates its great potential as promising candidate for the next generation photovoltaic cells. In this review article, we present a comprehensive overview of the development of QDSCs including: (1) fundamental principle, (2) brief evolution of QDSCs, (3) key materials in QDSCs, (4) recombination control, and (5) the stability issue. Finally, some directions that can further promote the development of QDSCs in the next future are proposed to help readers grasping the challenges and opportunities in achieving high efficiency QDSCs.
\end{abstract}




\section{Introduction}

Energy is a vital issue that humanity should be faced with at present and in the future. ${ }^{1}$ The great consumption amount of fossil energy has led to the crises of energy as well as the environment. Therefore, it is an urgent task to explore renewable clean energy to solve these problems. Among them, solar energy is believed to be the most promising renewable energy resource due to its fascinating characteristics such as inexhaustible and environmental friendly. In one year, the earth's surface can receive 3850 zettajoules (ZJ) from the sun, meaning that the solar energy absorbed by the earth's surface in one hour is larger than the whole world used in one year. ${ }^{2,3}$ There is no doubt that the rational utilization of solar energy is of great meaningful for humanity in the future. Solar cell is a device that provides the important advantage of converting solar energy directly into electric energy on the basis of photoelectric effect and can be used everywhere without the need of big facilities but also can be upscaled for massive energy production. Benefitting from the distinct characteristics of clean, low-cost, and abundant energy source, photovoltaic technology is considered to be the most promising one among all the renewable energy technologies. ${ }^{4}$

Since the appearance of the first crystalline silicon solar cell invited by Bell lab in 1954, solar cell technology has undergone a dramatically fast development. ${ }^{5}$ A variety of solar cells have been developed and parts of them have been realized the industry production. Typically, solar cells can be classified into three generations according to the materials and technology development. ${ }^{6,7}$ The first generation solar cell refers to silicon based solar cells, including monocrystalline and polycrystalline silicon cells. ${ }^{8-10}$ Until now, silicon solar cells are the most mature photovoltaic technology and dominate more than $90 \%$ of the photovoltaic market. The second generation solar cells are the thin film solar cell, including amorphous silicon, copper indium gallium selenide (CIGS), GaAs and CdTe etc. based devices. ${ }^{11-15}$ The direct band gap of these materials, in comparison with the indirect one from $\mathrm{Si}$, allows to harvest the light in thin films with very few micron thickness. Thin film solar cell takes advantage of cost reduction in the manufacturing process due to material saving and lower processing 
temperature. Even though the second generation solar cells exhibit comparable photovoltaic performance as silicon solar cells, they only possess a small market share $(<10 \%)$ due to the limitation of module technology and stability. The third generation solar cells are usually defined as emerging solar cells, most of which are still under the state of scientific research. ${ }^{4,16-23}$ The motivation of the exploration of the third generation solar cells is achieving higher efficiency solar cell with use of novel physical phenomena, materials and structures that can largely reduce the production cost. Currently, the third generation solar cells mainly include dye-sensitized solar cells (DSCs), organic/polymer solar cells (OSCs), perovskite solar cells (PSCs), and quantum dot (QD) based solar cells. ${ }^{4,16-23}$ In the past two decades, the third generation solar cells have attracted tremendous research interest and undergone fast development.
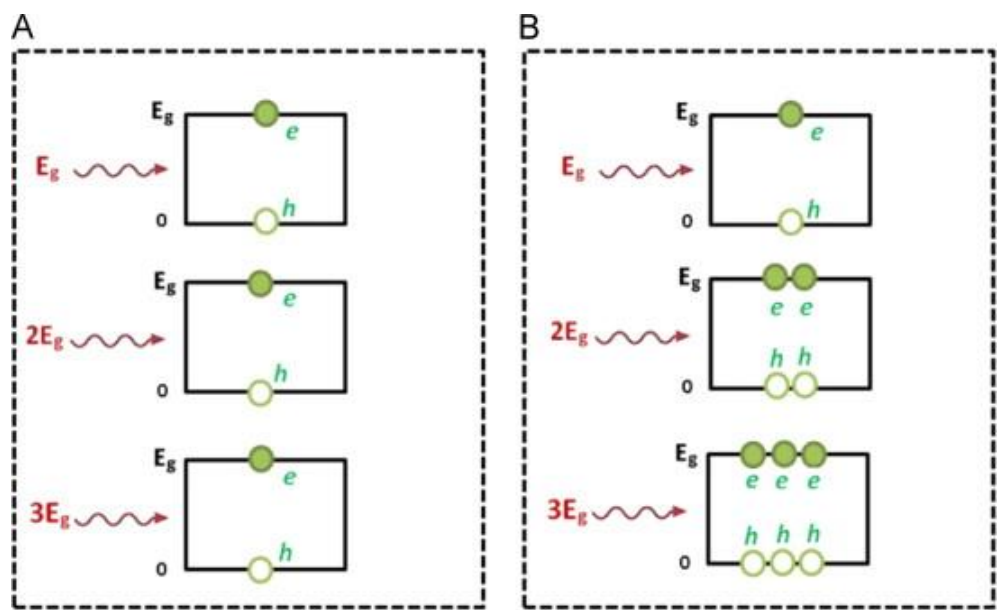

Fig. 1 Schematic illustration of the generation of carriers under the excitation of photon energy: (A) traditional solar cell and (B) ideal multiple exciton generation in QD based solar cell. Reprinted with permission from ref. 24. Copyright (2014) Elsevier.

In the study of the third generation solar cells, of particular interest was focused on QD based solar cell. ${ }^{19,20,25-27}$ This is motivated by the dramatic characteristics of semiconductor QD as light harvesting material, such as its facilely tunable band gap through size or composition control, high molar extinction coefficient, large intrinsic dipole moments, higher light, thermal, and moisture stability in comparison with dye molecules and lead halide perovsikes. Additionally, QDs can benefit of non-conventional properties, as shown in Fig. 1, 
as the multiple exciton generation (MEG) possibility, which could enable QD based solar cell overcoming the efficiency of Shockley-Quessier limit (32.9\%) for single absorber solar cells. $^{20,28}$

Typically, QD based solar cells can be classified into four kinds (Fig. 2): Schottky junction solar cell, p-n junction solar cell, hybrid QD-polymer solar cell, and quantum dot-sensitized solar cell (QDSCs). ${ }^{29-31}$ Among them, QDSCs possess the advantages of low-cost device fabrication process due to the simple device structure deriving from its analogous DSCs. ${ }^{24,26,30,32-42}$ It is noted that, during the past 12 years, the power conversion efficiency (PCE) of QDSCs has undergone a dramatically improvement from less than 1\% to nearly 13\% as shown in Fig. 3. The spectacular development trend of this kind of solar cell indicates its great potential as promising candidate for the next generation photovoltaic cells.

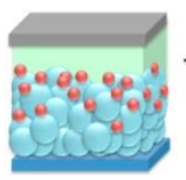

(a)

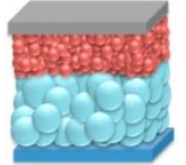

(d)
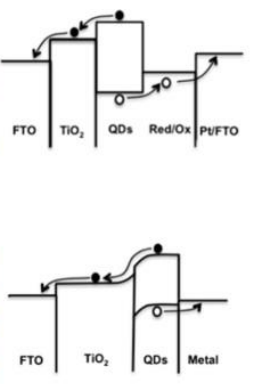

(e)

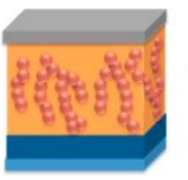

(b)

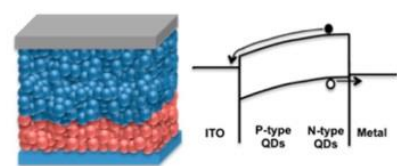

(e)
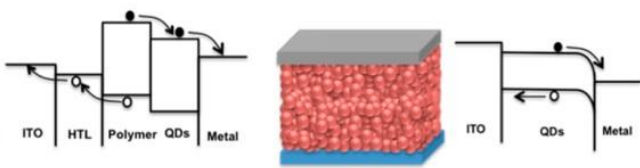

(c)

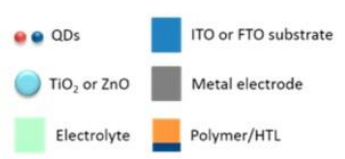

Fig. 2 Schematic illustration of device configurations and energy band diagram of QD-based solar cells: (a) QD-sensitized solar cell, (b) hybrid QD-polymer solar cell, (c) Schottky junction solar cell, (d) $\mathrm{p}-\mathrm{n}$ heterojunction solar cell, and (e) $\mathrm{p}-\mathrm{n}$ homojunction solar cell. Reprinted with permission from ref. 29. Copyright (2015) American Chemical Society.

In recent years, a series of reviews about QDSCs have been reported. Most of these review articles focused on single component of the cell device such as photoanode, electrolyte, and counter electrodes. ${ }^{24,26,30,32-53}$ While, a comprehensive overview about QDSCs, integrating fundamental principles, recent material and technical advances as well as the limitation for this kind of solar cell that should be overcome is still lacked. Hence, this review article presents a comprehensive overview of the integrated development of QDSC from its appearance to the current state. First, we review the fundamental working principle of 
QDSCs combining with the characterization techniques that commonly used. Second, a brief overview of the landmark works for QDSCs is presented. Third, we summarize the recent advances in key materials for QDSCs including metal oxide electron transporting material (ETM), light harvesting material QD sensitizers, counter electrode (CE) catalytic materials, and electrolyte redox couple or hole transporting materials (HTM). Then, we review the recent advances in charge recombination control in QDSCs, a critical factor in limiting the performance of QDSCs. Afterwards, we discuss the stability issue for QDSCs, which is usually neglected but very crucial for the development of this kind of photovoltaic technology. Finally, we propose some possible ways and issues that deserve to be addressed for promoting the development of QDSCs in the future furthermore.

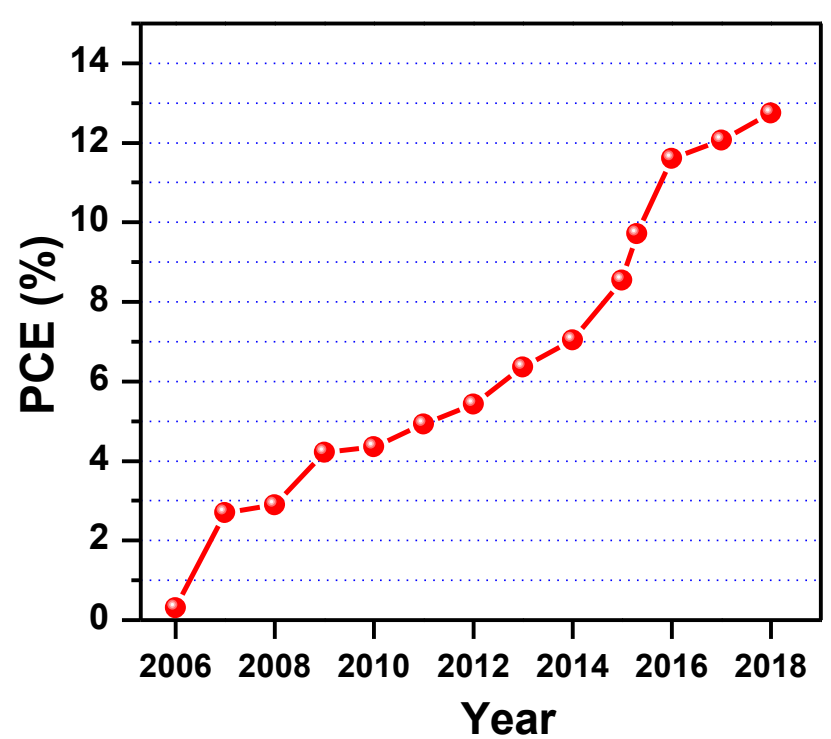

Fig. 3 Evolution of the record PCE of QDSCs since 2006 (for the cells based on standard two-electrode configuration and tested under the irradiation of AM 1.5G full one sun). The detailed data and the corresponding references are listed in Table 1. 


\section{Fundamental of QDSCs}

\subsection{Basic principles of QDSCs}

The concept of "sensitized semiconductor", in which a wide band gap semiconductor is sensitized with a narrow band gap semiconductor to harvest sunlight and generate charge carriers, can be dated back to $1960 \mathrm{~s} .{ }^{54}$ On the basis of this concept, the DSCs device was preliminarily studied and constructed as early as 1980 s. ${ }^{55}$ Initially, the smooth semiconductor electrodes were used for dye loading. The light harvesting efficiency of this flat electrode is very low due to the small dye loading amount, so that the efficiency of the cell device was very low. In 1991, O'Regan and Grätzel produced the first breakthrough of this technology by replacing the flat semiconductor electrode by a $\mathrm{TiO}_{2}$ mesoporous film, obtaining a significant improvement of the loading amount of dye and boosting the PCE of DSCs to $7.1 \% .{ }^{56}$ From that time, DSCs drew much attention from all over the world, and therefore the DSC is also called "Grätzel cell". ${ }^{16,57-62}$ The pioneering work by Grätzel and coworkers is also regarded as a milestone for the beginning of the investigation of the third-generation PV technology.

The great contribution of the sensitized cells is that they decouple the charge generation and the charge transport. In silicon or in thin film solar cells, both processes occur in the same material and consequently the quality of materials has to be high in order to avoid defects promoting the recombination. In sensitized devices, charge generation is produced in the dye while photogenerated charge is quickly injected into two different transport media. Thus, a medium only transports a type of carriers, decreasing consequently recombination and allowing that the high standards of quality of materials can be relaxed and therefore manufacturing costs reduced. ${ }^{63}$

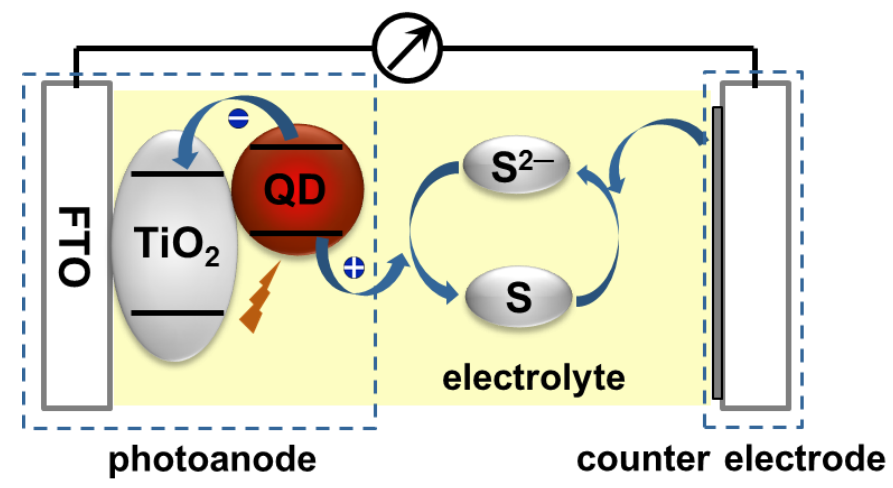

Fig. 4 Schematic illustration of the device structure of QDSCs. 
The architecture of QDSCs is directly derived from DSCs, using QDs to replace organic dye molecules as the light harvesting material. ${ }^{43}$ The investigation of QDSCs is initially motivated by the attractive photoelectronic properties of QDs. ${ }^{20,26,64}$ Typically, as shown in Fig. 4, a QDSC is composed of a QD sensitized photoanode, electrolyte, and a counter electrode..$^{30,37,45}$ Upon light irradiation, QD absorbs solar energy and electrons in the valence band (VB) of QDs are excited to the conduction band (CB), generating electron-hole pairs. Then, electrons in the CB of QD quickly injected into the CB of metal oxide (generally $\mathrm{TiO}_{2}$ ) electron acceptor under the driving force of the energetic difference of CB between QD and metal oxide, achieving charge separation process. The electrons transfer through the $\mathrm{TiO}_{2}$ mesoporous film to transparent conductive oxide substrate and then get to counter electrode through external circuit. Meanwhile, the oxidized QDs are regenerated by reduced species of the redox couple in the electrolyte and the oxidized species of redox couple are then reduced by the electrons from external circuit under the catalysis of CE. Apart from these desired charge transport processes, some other unwanted processes, also known as charge recombination, will take place simultaneously and significantly deteriorates solar cell performance. ${ }^{33,38}$ This will be discussed in detail in the following section.

\subsection{Characterizations of QDSCs}

In order to evaluate the photovoltaic performance of the cell device and get insight into the charge transfer dynamics at the interfaces, such as the charge separation and recombination processes, a variety of characterization methods have been developed for QDSCs. Among them, current-voltage $(J-V)$, incident photon conversion efficiency (IPCE), absorbed photon to current conversion efficiency (APCE), time resolved photoluminescence (TRPL), transient absorption (TA), electrochemical impendence spectroscopy (IS), open-circuit voltage-decay (OCVD), and charge extraction measurements are the most widely used characterization routes in QDSCs.

2.2.1. Photovoltaic performance measurements. Similar to all kinds of solar cells, the photovoltaic performance measurements of QDSCs consist of $J-V$ and IPCE tests. The $J-V$ curve is usually used to determine the PCE of the solar cell, showing the relationship between the output current and voltage of the solar cell under irradiation. From a $J-V$ curve (Fig. 5), we can directly obtain the short circuit current density $\left(J_{\mathrm{sc}}\right)$ and the open-circuit voltage $\left(V_{\mathrm{oc}}\right)$ values on the basis of the intercepts of the $J-V$ curve in the lateral and vertical axes, respectively. The $J_{\mathrm{sc}}$ and $V_{\mathrm{oc}}$ values represent the maximum photocurrent and photovoltage output capability of the cell, respectively. In a $J-V$ curve, there exists a point that the product 
of photocurrent $\left(I_{\mathrm{mp}}\right)$ and photovoltage $\left(V_{\mathrm{mp}}\right)$, namely the output power $\left(P_{\max }\right)$ is maximum. The specific value of $P_{\max }$ to the product of $V_{\mathrm{oc}}$ and $J_{\mathrm{sc}}$ is defined as the fill factor (FF), which is in the range from 0 to 1 . The PCE of the QDSCs can be calculated as follows:

$$
\mathrm{PCE}=\frac{I_{\mathrm{mp}} \cdot V_{\mathrm{mp}}}{P_{\mathrm{in}}}=\frac{J_{\mathrm{sc}} \cdot V_{\mathrm{oc}} \cdot \mathrm{FF}}{P_{\mathrm{in}}}
$$

where $P_{\text {in }}$ is the power density of the incident light.

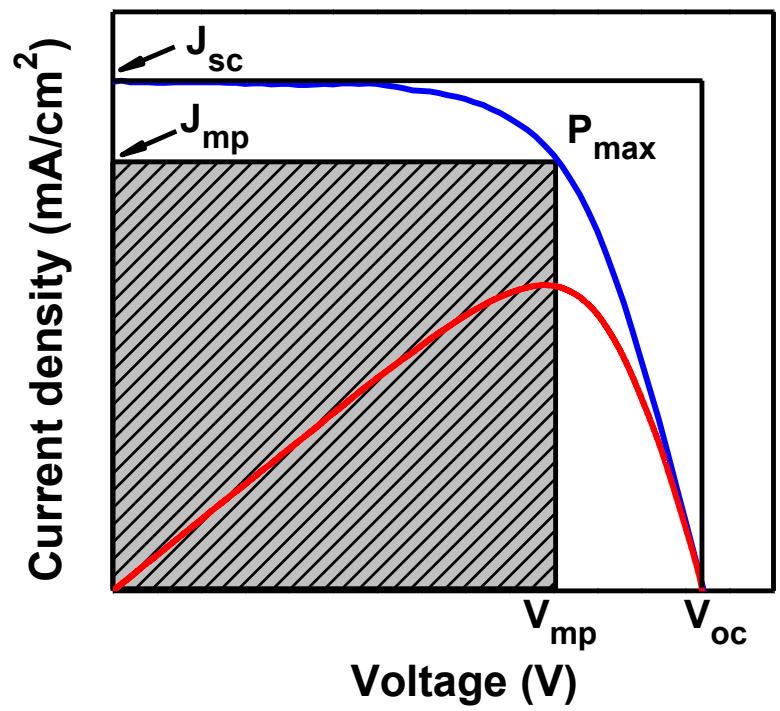

Fig. $5 J-V$ characteristic curves of QDSCs.

It should be noted that $J-V$ measurement conditions should be strict and standard to get credible results. ${ }^{65}$ This is very crucial to make a comparison of the results from different labs. For example, a proper black mask should be used in the measurement to define the active area and avoid excess light into the QDSCs. What's more, the spectrum of the light source should be exactly the same as the solar spectrum (AM 1.5G) and a Si standard solar cell should be used to calibrate the intensity of the light $(100 \mathrm{~mW} \mathrm{~cm}$, one sun). To render the photovoltaic performances reported by different laboratories are comparable, a certified PCE by a third party is therefore very important, especially for record devices defining a new state-of-the art. In addition, some other parameters, such as the scan rate, direction, and delay time should also be carefully taken into account during the measurement. ${ }^{65}$ It is noted that unlike the case for perovskite solar cells, the hysteresis in $J$ - $V$ measurements was barely observed in the QDSCs.

A solar cell can be seen as an equivalent circuit that consists of a light generated current source, diode and series resistance. ${ }^{65}$ Normally, there are two approaches resulting power 
dissipation, which are the leakage current form shunt (parallel) resistance $\left(R_{\text {shunt }}\right)$ and the voltage loss form series resistance $\left(R_{\text {series }}\right)$. The $R_{\text {shunt }}$ and $R_{\text {series }}$ values of device can be obtained from the slope $J-V$ curve at the voltages of $0 \mathrm{~V}$ and $V_{\mathrm{oc}}$, respectively. These two parameters have a great influence on the FF value of a solar cell, and a large $R_{\text {shunt }}$ and a small $R_{\text {series }}$ will promise a high FF. Generally, the recombination reduction can decrease the

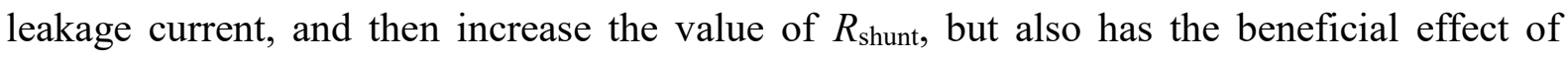
increase open-circuit potential. Besides, it is helpful to decrease the resistance of the contacts and during charge transport, in order to reduce $R_{\text {series. }}{ }^{66}$

Another important photovoltaic performance measurement route in QDSCs is the IPCE, also known as external quantum efficiency (EQE), corresponding to the number of generated electrons $\left(N_{\mathrm{e}}\right)$ divided by the number of incident monochromatic photons $\left(N_{\mathrm{p}}\right){ }^{7,24,45}$ The IPCE can be expressed as follows:

$$
\operatorname{IPCE}(\lambda)=\frac{N_{\mathrm{e}}}{N_{\mathrm{p}}}=\frac{1240 \cdot J_{\mathrm{sc}}(\lambda)}{\lambda \cdot P_{\text {in }}}
$$

where $J_{\mathrm{sc}}$ is the short-circuit current density under monochromatic light illumination (with wavelength $\lambda$ ) and $P_{\text {in }}$ is the power density of incident light. IPCE measurement is usually used to confirm photoresponse range of the device as well as the $J_{\mathrm{sc}}$ value obtained from $J-V$ test. A $J_{\mathrm{sc}}$ value can also be obtained by integrating the product of the incident photon flux density $(\mathrm{F})$ and the measured IPCE values as follows: ${ }^{45}$

$$
J_{\mathrm{sc}}=\int q \mathrm{~F}(\lambda) \operatorname{IPCE}(\lambda) \mathrm{d}(\lambda)
$$

where $q$ is the electron elementary charge. The $J_{\text {sc }}$ values obtained from the IPCE and $J-V$ tests should be identical for an ideal solar cell, and it is an effective test of the correct measurement of photocurrent. In addition, the IPCE value is affected by the light harvesting efficiency (LHE), electron injection efficiency from QD to $\mathrm{TiO}_{2}\left(\varphi_{\text {inj }}\right)$, and charge collection efficiency $\left(\varphi_{\mathrm{cc}}\right)$. Therefore, IPCE can also be described as follows:

$$
\operatorname{IPCE}(\lambda)=\operatorname{LHE}(\lambda) \cdot \varphi_{\text {inj }} \cdot \varphi_{\mathrm{cc}}
$$

Absorbed photon to current conversion efficiency (APCE) is also called as the internal quantum efficiency (IQE), corresponding to the number of generated electrons $\left(N_{\mathrm{e}}\right)$ divided by the number of absorbed photons $\left(N_{\mathrm{a}}\right)$. The APCE results can reflect the efficiency of absorbed photons by QD that converted into photocurrent, excluding the effect of light harvesting efficiency of the photoanode. ${ }^{24,65}$ The APCE can be described as the following 
equation:

$$
\operatorname{APCE}(\lambda)=\frac{N_{\mathrm{e}}}{N_{\mathrm{a}}}=\frac{\operatorname{IPCE}(\lambda)}{\operatorname{LHE}(\lambda)}=\varphi_{\text {inj }} \cdot \varphi_{\mathrm{cc}}
$$

\subsubsection{Time resolved photoluminescence (TRPL) and ultrafast transient absorption}

(TA) measurements. TRPL and ultrafast TA spectroscopy are usually used to monitor the dynamics of charge transfer processes in QDSCs, including electron injection rate from QDs to metal oxide, hole transfer rate from oxidized QDs to electrolyte, and the charge recombination rate at photoanode/electrolyte interface. T. Pullerits et al. have made outstanding efforts in this regard, whose works elaborated the related mechanism of the charge dynamics in QDSCs. ${ }^{67-72}$

In the TRPL measurement, a pulsed laser with a high frequency is applied to excite samples. Typically single photon counting is used to produce a statistics of the decay time. Each laser pulse the detector waits until the first emitted photon arrives, recording the delay between excitation and emission. The high frequency allows obtaining the PL decay behavior after the accumulation of enough detected events. Then, the corresponding excited state lifetime is obtained by fitting the decay curves of photoluminescence. ${ }^{73,74}$ For the sample of QDs in solution or attached to isolating semiconductor films, its photo-excited electrons directly decay back to the ground state through radiative or non-radiative recombination processes. As a result, this lifetime $(\tau)$ determined from the decay curve reveals the recombination dynamics of the QDs without charge transfer processes. For the sample of QDs attached to electron transporting metal oxide films, such as $\mathrm{TiO}_{2}$ and $\mathrm{ZnO}$, the electrons in excited state can be extracted out via the charge injection process. Herein, the lifetime $\left(\tau^{\prime}\right)$ exhibits the dynamics of charge transfer processes in QDSCs. The decay curve can be fitted with a multiple-exponential function of the following equation:

$$
\mathrm{y}=\sum_{\mathrm{i}=1}^{\mathrm{n}} A_{\mathrm{n}} \exp \left(-t / \tau_{\mathrm{n}}\right)
$$

where $\tau_{\mathrm{n}}$ and $A_{\mathrm{n}}$ are lifetimes and weighted coefficients for the multiple-components, respectively. In QDSCs, the commonly approach is a biexponential function $(n=2)$. For the multiple components process, an average lifetime $\left(\tau_{\mathrm{av}}\right)$ of photoexcited electrons can be calculated using the following equation:

$$
\tau_{\mathrm{av}}=\sum_{\mathrm{i}=1}^{\mathrm{n}} A \tau_{\mathrm{i}}^{2} / \sum_{\mathrm{i}=1}^{\mathrm{n}} A \tau_{\mathrm{i}}
$$

In principal, the charge injection rate constant $\left(K_{\mathrm{et}}\right)$ can be calculated according to the 
obtained average lifetimes ( $\tau_{a v}$ and $\left.\tau_{a v}{ }^{\prime}\right)$ using the following equation:

$$
K_{\mathrm{et}}=1 / \tau_{\mathrm{av}}{ }^{\prime}-1 / \tau_{\mathrm{av}}
$$

Moreover, the ultrafast TA spectroscopy also plays a significant role in revealing the charge transfer processes in most of the emerging solar cells. ${ }^{32,39,65,75-80}$ The schematic diagram of ultrafast TA equipment is shown in Fig. 6. In this measurement, the pulses with a certain frequency are equipped by a femtosecond Ti-sapphire laser and then split into pump and probe pulses. The wavelength of pump pulses is modulated by optical parametric amplifier (OPA) and a series of neutral-density filters was explored to adjust the power of the pump beam. Meanwhile, the initial probe of monochromatic light will be transformed into a white light after passing the crystal of sapphire or calcium fluoride. The pump and probe pulses focus at the same point of samples with a certain angle and different delay times. The delay times $(\Delta t)$ between of pump and probe pulses can be adjusted through a motorized delay stage. A detector is used to record the absorption $\left(A_{\mathrm{t}}\right)$ at different delay time $(\Delta t)$ after pump pulse and the absorption $\left(A_{0}\right)$ without pump pulse. The induced absorbance change $(\Delta A)$ can be calculated through $A_{\mathrm{t}}$ minus $A_{0}$. Since we can measure the TA spectra at different pumpprobe delay times, the $\Delta A$ can be determined as a function of both wavelength and time. Finally, the charge lifetimes are given by fitting with decay curves of $\Delta A$ versus time similar to the TRPL measurement.

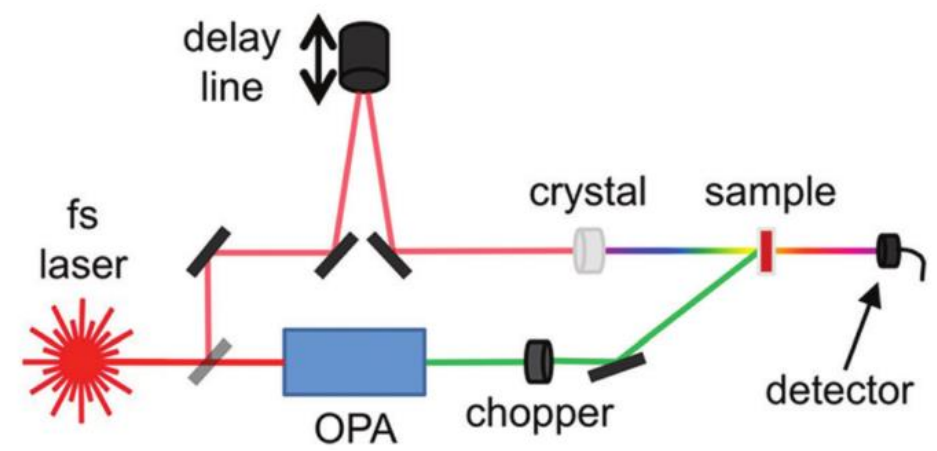

Fig. 6 Schematic diagram of the main components in an ultrafast TA equipment. Reprinted with permission from ref. 65. Copyright (2017) Royal Society of Chemistry.

2.2.3. Impendence spectroscopy (IS) measurement. IS allows to decoupling physical processes occuring at different time (frequency) scales. In this sense, it is very interesting for the study of complete device at the working conditions, as information of the different parts 
can be extracted from a single measurement. ${ }^{33,65,66,81}$ IS allows to study the kinetics of charge transport and recombination at $\mathrm{TiO}_{2} / \mathrm{QD} /$ electrolyte interfaces. In addition, also permits to evaluate the catalytic activity of CEs in QDSCs. In this measurement, a DC voltage is applied to the system and a small AC signal is used to perturb the system simultaneously. Afterwards, the sinusoidal current response is measured as a function of modulation frequency. According to the applied perturbation and the measured response, the impedance value can be obtained. On the basis of well-developed equivalent circuit, the electrochemical parameters such as series resistance $\left(R_{\mathrm{S}}\right)$, charge transfer resistance $\left(R_{\mathrm{ct}}\right)$, recombination resistance $\left(R_{\mathrm{rec}}\right)$ and diffusion resistance $\left(Z_{\mathrm{w}}\right)$ can be obtained by fitting the data with software. ${ }^{66,81}$ The IS results are often expressed in a complex plane plot (imaginary part of impedance vs. real part) or a Bode plot showing the parameter of interest vs. frequency. Typically, two semicircles can be found in a complex plane impedance plot for QDSCs. The $R_{\mathrm{S}}$ can be obtained from the starting point of the plot. The first semicircle at high frequency is assigned to the $R_{\mathrm{ct}}$ at $\mathrm{CE} /$ electrolyte interface and the second semicircle at middle and low frequency refers to the $R_{\mathrm{ct}}$ or $R_{\text {rec}}$, depending on the applied bias, at the $\mathrm{TiO}_{2} / \mathrm{QD} /$ electrolyte interfaces. Finally, the electrochemical properties of the materials or the interfaces can be analyzed according to the obtained electrochemical parameters.

2.2.4. Open-circuit voltage-decay (OCVD) measurement. The OCVD measurement is an alternative tool that commonly used to obtain the electron lifetime $\left(\tau_{\mathrm{n}}\right)$ of excited electrons in DSCs or QDSCs. ${ }^{82,83}$ Typically, in this measurement, the cell is firstly illuminated under open-circuit conditions for a certain period to get to a steady $V_{\text {oc. }}$ Then, the light is turned off and the output $V_{\text {oc }}$ is traced as a function of time. The $V_{\text {oc }}$ decay rate is directly relevant to the electron lifetime of the cell since the variation of $V_{\text {oc }}$ after turning off the light is determined by the lifetime of photogenerated electrons. The corresponding $\tau_{\mathrm{n}}$ can be calculated according to the following equation:

$$
\tau_{\mathrm{n}}=-\frac{-k_{\mathrm{B}} T}{e}\left(\frac{\mathrm{d} V_{\mathrm{oc}}}{\mathrm{d} t}\right)^{-1}
$$

where $k_{\mathrm{B}}$ is the Boltzmann constant, $T$ is the absolute temperature $(298.15 \mathrm{~K})$, and $e$ is the elementary charge. The OCVD test can be used to estimate the whole degree of charge recombination in QDSCs. During the measurement, once the light is turned off, the decay of the $V_{\text {oc }}$ is determined by the rate constant of charge recombination at $\mathrm{TiO}_{2} / \mathrm{QD} /$ electrolyte interfaces. Therefore, we can evaluate the charge recombination dynamics in the solar cells according to the calculated electron lifetime from the decay curves. 
2.2.5. Charge extraction measurement. For DSCs and QDSCs, the charge extraction measurement is usually applied to indicate the shift of the Fermi level of electron transporting metal oxide, an important factor affecting the $V_{\text {oc }}$ of devices. ${ }^{84-86}$ At the beginning of the measurement, the device is illuminated and kept at open-circuit conditions. Afterwards, the light is switched-off and then the device is switched to short-circuit conditions with a delay time. Meanwhile, the current is recorded and integrated versus time to obtain the amount of extracted charge $\left(Q_{\mathrm{oc}}\right)$. The obtained $Q_{\mathrm{oc}}$ can reflect the density states of the metal oxide film electrode and therefore we can estimate the shift of its Fermi level. 


\section{Brief evolution of QDSCs}

Dating from 1970s, researchers found that the efficiency of charge separation can be increased by contacting the semiconductor particle with another kind of semiconductor. ${ }^{87-94}$ For example, in 1984, Grätzel et al. demonstrated that the combination of $\mathrm{CdS}$ and $\mathrm{TiO}_{2}$ can increase the yield of $\mathrm{H}_{2}$ generation from $\mathrm{H}_{2} \mathrm{~S}$ benefitting from the electron transfer from the conduction band of CdS to that of $\mathrm{TiO}_{2}$ particles. ${ }^{87}$ Gerischer et al. introduced the concept of dye sensitization to semiconductor sensitization. They deposited $\mathrm{CdS}$ on $\mathrm{TiO}_{2}$ and found that photocurrent of the electrode was improved due to the extended light absorption range. ${ }^{88}$ These works established the initial concept of the present QDSCs. Since then, many kinds of light harvesting semiconductors, such as $\mathrm{CdSe}, \mathrm{PbS}, \mathrm{CdS}$, and $\mathrm{Bi}_{2} \mathrm{~S}_{3}$ et al., were used to sensitize wide band gap semiconductors and investigated their photo-electrochemical properties. $^{94}$

In 1990, Weller and coworkers sensitized highly porous $\mathrm{TiO}_{2}$ electrode by quantum sized CdS particles through in situ deposition approach. ${ }^{91}$ They also fabricated a three-electrode based photoelectrochemical cell and obtained a $V_{\text {oc }}$ of $0.395 \mathrm{~V}$, a $J_{\mathrm{sc}}$ of $175 \mathrm{~mA} / \mathrm{m}^{2}$, and a FF of 0.75 under the illumination of a monochromatic light with wavelength of $460 \mathrm{~nm}$. Until 1998, Zaban and Nozik et al. deposited pre-synthesized InP QDs on $\mathrm{TiO}_{2}$ electrode and fabricated a sandwich-type QDSC device for the first time with use of $\mathrm{I}^{-} / \mathrm{I}_{3}{ }^{-}$as electrolyte and Pt as counter electrode. ${ }^{95}$ The photocurrent of the constructed cell device was monitored under the illumination of incident light. This is the first report of the integral QDSCs device, even though the final PCE of the solar cell was not given. Afterwards, InAs QDs with different sizes were also used as sensitizer by Nozik's group and an efficiency of $0.3 \%$ was obtained. ${ }^{96}$ Considering the limited stability of QDs in $\mathrm{I}^{-} / \mathrm{I}_{3}^{-}$electrolyte, Grätzel et al. constructed the first solid-state QDSC employing 2,2',7,7'-tetrakis(N,N-di-p-methoxyphen ylamine)-9,9'-spirobifluorene) (spiro-OMeTAD) as the hole transport material (HTM) and obtained an efficiency of $0.49 \%$ under 0.1 sun illumination in $2002 .{ }^{97}$ Until 2006, the QDSCs didn't draw much attention since the obtained efficiency was much lower in comparison with its analogue DSCs. ${ }^{98}$

The first leap of QDSCs was achieved by Toyoda and coworkers. ${ }^{99}$ The PCE of QDSCs was boosted to over $2 \%$ in 2007 by Toyoda et al. In this work, they fabricated CdSe sensitized $\mathrm{TiO}_{2}$ inverse opal solar cells combining with a polysulfide electrolyte. What's more, the authors demonstrated that a $\mathrm{ZnS}$ thin layer overcoating on photoanode can 
significantly improve the solar cell performance. It should be noted that this $\mathrm{ZnS}$ treatment has been evolved as an indispensable procedure in the fabrication of high efficiency QDSCs since then. After that, Lee et al. introduced a two-step QDs deposition approach, in which a pre-synthesized $\mathrm{CdS}$ seed layer were first self-assembled on $\mathrm{TiO}_{2}$ electrode surface using a bifunctional linker molecule, followed by a chemical bath deposition (CBD) process for the growth of CdSe layer. This configuration achieved a PCE of $2.8 \%{ }^{100}$ Then, Lee and coworkers put forward a classical $\mathrm{CdS} / \mathrm{CdSe}$ co-sensitization structure through successive ionic layer adsorption and reaction (SILAR) deposition approach, boosting the PCE of QDSCs to $4.22 \%$ in $2009 .{ }^{101}$ Grätzel et al. developed a new procedure for preparing selenide $\left(\mathrm{Se}^{2-}\right)$ source by reducing the corresponding dioxide precursor in ethanol and used for depositing CdSe QDs on $\mathrm{TiO}_{2}$ through SILAR approach, exhibiting a PCE of $4.18 \%$ under 0.1 sun illumination using a cobalt redox couple. ${ }^{102}$

As of 2009, QDSCs drew more and more attention due to the significantly improved photovoltaic performance. Fan et al. explored ordered multimodal porous carbon (OMPC) and mesoporous carbon nanofibers (MCNFs) as counter electrode catalytic materials in QDSCs and achieved efficiencies of $4.36 \%$ and $4.81 \%$, respectively. ${ }^{103,104} \mathrm{Xu}$ et al. designed a $\mathrm{ZnO} / \mathrm{Zn}_{\mathrm{x}} \mathrm{Cd}_{1-\mathrm{x}} \mathrm{Se}$ core/shell nanocable array photoanode, delivering a PCE of $4.74 \% .{ }^{105}$ Meng et al. systematically optimized the structure of $\mathrm{TiO}_{2}$ film electrode based on $\mathrm{CdS} / \mathrm{CdSe}$ QDs system, giving a best efficiency of $4.92 \% .{ }^{106}$ Kamat et al. proposed a doping strategy in QDs sensitizers to facilitate charge transfer and charge separation processes. They employed $\mathrm{Mn}^{2+}$ doping in CdS layer and prepared Mn-doped-CdS/CdSe QDs sensitizers, boosting the PCE of QDSCs over 5\% (5.4\%) for the first time. ${ }^{107}$

Since 2012, we have been witnessing a rapid evolution of the record PCE of QDSCs arising from the development of both material and mechanism investigations. Until this year the highest efficiencies were obtained with sensitized electrodes where QDs where directly grown on the mesoporous electrode by CBD or SILAR method. However, the characterization of devices using QDs prepared in different ways pointed out a better potentiality for devices using colloidal QDs as sensitizers. ${ }^{108}$ Nevertheless the efficiency of QDSCs using colloidal QDs was limited by the low QD loading that limited the light harvesting. In 2012, Zhong's group focused on the utilization of high quality pre-synthesized colloidal QDs as sensitizers, and deposited QDs on $\mathrm{TiO}_{2}$ electrode through the capping ligand induced self-assembly (CLIS) approach developing the capping ligand induced self assembly (CLIS) approach to deposit QDs on $\mathrm{TiO}_{2}$-electrode. ${ }^{109,110}$ This novel deposition method was 
proven to be capable of realizing fast, uniform and high loading amount of colloidal QDs on $\mathrm{TiO}_{2}$ electrode. ${ }^{74}$ What's more, the CLIS approach provides a way to introduce high quality QDs as sensitizer in QDSCs, and since that moment the successive efficiency records were obtained with colloidal QDs as sensitizers. ${ }^{36,74,85,109-119}$ Through the exploration of superior colloidal QDs sensitizers and interface modification engineering, the highest PCE of QDSCs was improved from $5 \%$ to nearly $13 \%$ during the past 5 years, leading to a huge step forward in competing with other kinds of emerging solar cells. The evolution of the PCE for QDSCs is displayed in Fig. 3 and the corresponding works are listed in Table 1.

Table 1 Summary of the landmark works in the evolution of QDSCs (for the cells based on standard two-electrode configuration with use of $\mathrm{TiO}_{2}$ based photoanodes and tested under the irradiation of AM $1.5 \mathrm{G}$ full one sun).

\begin{tabular}{|c|c|c|c|c|c|c|c|c|}
\hline year & QDs & $\mathbf{C E}$ & electrolyte & $J_{\mathrm{sc}}\left(\mathrm{mA} \mathrm{cm}{ }^{-2}\right)$ & $V_{\text {oc }}(\mathrm{V})$ & FF & PCE (\%) & Ref. \\
\hline 1998 & $\operatorname{InP}$ & $\mathrm{Pt}$ & $\mathrm{I}^{-} / \mathrm{I}_{3}^{-}$ & - & - & 0.685 & - & 95 \\
\hline 2002 & $\mathrm{PbS}$ & - & spiro-OMeTAD & - & 0.24 & - & 0.49 & 97 \\
\hline 2007 & $\mathrm{CdSe}$ & $\mathrm{Pt}$ & polysulfide & 7.51 & 0.71 & 0.50 & 2.7 & 99 \\
\hline 2008 & $\mathrm{CdS} / \mathrm{CdSe}$ & $\mathrm{Au}$ & polysulfide & 11.66 & 0.503 & 0.49 & 2.9 & 100 \\
\hline 2009 & $\mathrm{CdS} / \mathrm{CdSe}$ & $\mathrm{Au}$ & polysulfide & 16.8 & 0.514 & 0.49 & 4.22 & 101 \\
\hline 2011 & $\mathrm{CdS} / \mathrm{CdSe}$ & $\mathrm{Cu}_{2} \mathrm{~S} /$ brass & polysulfide & 13.68 & 0.575 & 0.63 & 4.92 & 106 \\
\hline 2012 & $\mathrm{Mn}-\mathrm{CdS} / \mathrm{CdSe}$ & $\mathrm{Cu}_{2} \mathrm{~S}-\mathrm{RGO}^{\mathrm{a}}$ & polysulfide & 20.7 & 0.558 & 0.47 & 5.42 & 107 \\
\hline 2013 & $\mathrm{CdSe}_{\mathrm{x}} \mathrm{Te}_{1-\mathrm{x}}$ & $\mathrm{Cu}_{2} \mathrm{~S} /$ brass & polysulfide & 19.35 & 0.571 & 0.575 & 6.36 & 111 \\
\hline 2013 & $\mathrm{CdTe} / \mathrm{CdSe}$ & $\mathrm{Cu}_{2} \mathrm{~S} / \mathrm{brass}$ & polysulfide & 19.59 & 0.606 & 0.569 & 6.76 & 74 \\
\hline 2014 & $\mathrm{CuInS}_{2}$ & $\mathrm{Cu}_{2} \mathrm{~S} /$ brass & polysulfide & 20.65 & 0.586 & 0.581 & 7.04 & 116 \\
\hline 2015 & $\mathrm{CdSe}_{\mathrm{x}} \mathrm{Te}_{1-\mathrm{x}}$ & $\mathrm{Cu}_{2} \mathrm{~S} / \mathrm{brass}$ & polysulfide & 20.78 & 0.653 & 0.605 & 8.21 & 117 \\
\hline 2015 & $\mathrm{CdSe}_{\mathrm{x}} \mathrm{Te}_{1-\mathrm{x}}$ & $\mathrm{Cu}_{\mathrm{x}} \mathrm{S} / \mathrm{FTO}$ & polysulfide & 20.78 & 0.702 & 0.636 & 9.28 & 113 \\
\hline 2016 & $\mathrm{CdSe}_{\mathrm{x}} \mathrm{Te}_{1-\mathrm{x}}$ & $\mathrm{Cu}_{2} \mathrm{~S} /$ brass & polysulfide & 21.04 & 0.720 & 0.642 & 9.73 & 114 \\
\hline 2016 & $\mathrm{CdSe}_{\mathrm{x}} \mathrm{Te}_{1-\mathrm{x}}$ & $\mathrm{MC}^{\mathrm{b}} / \mathrm{Ti}$ & polysulfide & 20.69 & 0.807 & 0.689 & 11.51 & 118 \\
\hline 2016 & ZCISe & $\mathrm{MC} / \mathrm{Ti}$ & polysulfide & 25.49 & 0.745 & 0.627 & 11.91 & 115 \\
\hline 2017 & ZCISe & $\mathrm{N}-\mathrm{MC} / \mathrm{Ti}$ & polysulfide & 25.67 & 0.759 & 0.639 & 12.45 & 119 \\
\hline 2018 & ZCISe-CdSe & $\mathrm{MC} / \mathrm{Ti}$ & polysulfide & 27.39 & 0.752 & 0.619 & 12.75 & 112 \\
\hline
\end{tabular}

${ }^{\mathrm{a}} \mathrm{RGO}=$ reduced graphene oxide, ${ }^{\mathrm{b}} \mathrm{MC}=$ mesoporous carbon 


\section{Key materials}

\subsection{Metal oxide electron transporting/acceptor material (ETM)}

Metal oxide electron transporting/acceptor (ETM) materials are used to construct the substrate of photoanode, which is the scaffold for the deposition of QD sensitizers in photoanode. The ETM performs the function of supporting QDs, extracting photo-excited electrons from QD and transporting them to the conductive substrate (usually FTO). ${ }^{42,47,50,120}$ Thus, the preferred electron transporting film should possess the following properties: (1) appropriate conduction band edge, which will determine the photogenerated electron extraction efficiency as well as the $V_{\text {oc }}$ of the cell device; (2) high electron mobility to facilitate the extracted electron transfer in the film and collected by the conductive substrate; (3) abundant surface area to afford sufficient QDs loading amount for efficient light harvesting; (4) chemical stability, low toxicity and simple preparation. Accordingly, studies on electron transporting materials in QDSCs mainly focus on these crucial properties. Among these, $\mathrm{TiO}_{2}$ and $\mathrm{ZnO}$ are the most widely studied ETMs in QDSCs. The representative photovoltaic performances for QDSCs based on different ETMs are summarized in Table 2.

4.1.1. $\mathrm{TiO}_{2}$ based photoanode. $\mathrm{TiO}_{2}$ based nanomaterial is believed to be a suitable ETM due to its distinguishing advantages including good chemical stability, nontoxicity, and low cost. ${ }^{50,120}$ Among them, $\mathrm{TiO}_{2}$ nanoparticle $\left(\mathrm{TiO}_{2}-\mathrm{NP}\right)$ based mesoporous films are the most commonly used photoanode in DSCs and QDSCs due to its large surface area, facile fabrication and good stability. ${ }^{120}$ It is noted that most of the high efficiency QDSCs ever reported were derived from $\mathrm{TiO}_{2}-\mathrm{NP}$ (commonly P25) based ETM. ${ }^{115,117-119}$ Nevertheless, it is well known that the electron transport in NP based film is unordered, resulting in a long electron transport length to the conductive substrate and therefore increasing the charge recombination probability. Thus, aiming at realizing a faster electron transport channel, one-dimensional (1D) structured $\mathrm{TiO}_{2}$, such as nanotube $\left(\mathrm{TiO}_{2}-\mathrm{NT}\right),{ }^{121-131}$ nanorod $\left(\mathrm{TiO}_{2}-\mathrm{NR}\right),{ }^{132-140}$ and nanowire $\left(\mathrm{TiO}_{2}-\mathrm{NW}\right)^{141-145}$ have been explored as ETM in QDSCs to facilitate faster electron transfer to conductive substrate. It is believed that the 1D structure of ETM can provide a smoother electron transport tunnel and thus decrease the recombination loss. However, the reduction of effective area of the electrode decreases the QD loading amount, decreasing the light harvesting capability, see below. 


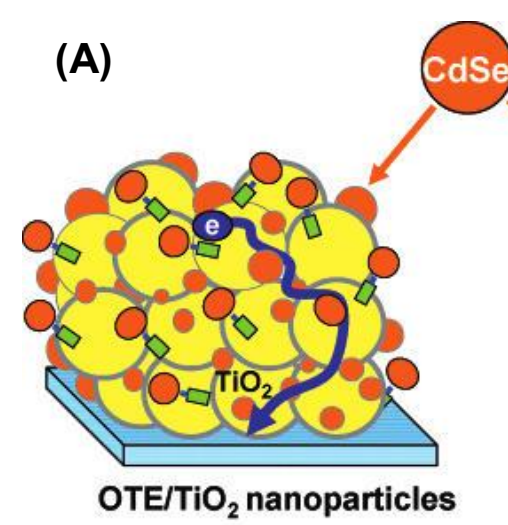

(a)

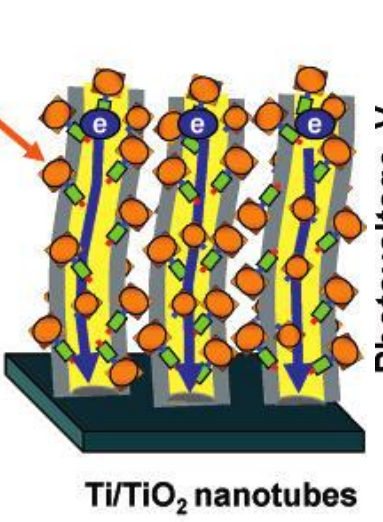

(b)

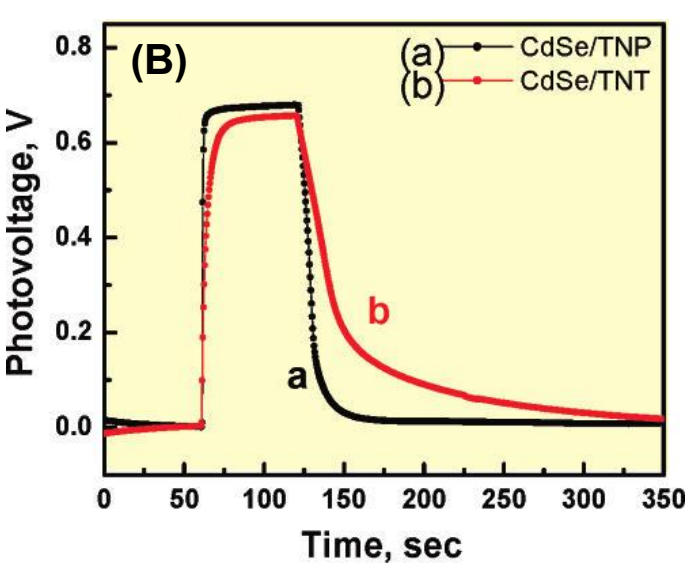

Time, sec

Fig. 7 (A) Random versus directed electron transport through support architectures, (a) $\mathrm{TiO}_{2}$ particle and (b) $\mathrm{TiO}_{2}$ nanotube films modified with CdSe quantum dots. (B) Open-circuit photovoltage response of (a) $\mathrm{OTE} / \mathrm{TiO}_{2}$ (particle) $/ \mathrm{CdSe}$ and (b) $\mathrm{Ti} / \mathrm{TiO}_{2}$ (nanotube) $/ \mathrm{CdSe}$ electrodes using $3.0 \mathrm{~nm}$ CdSe quantum dots as light absorbers. Reprinted with permission from ref. 73. Copyright (2008) American Chemical Society.

As early as 2007 , Kamat et al. have shown that the $\mathrm{TiO}_{2}-\mathrm{NT}$ is superior to $\mathrm{TiO}_{2}-\mathrm{NP}$ as ETM in constructing QDSCs due to the better electron transport capacity, since the large grain boundaries in $\mathrm{TiO}_{2}$-NP based film will increase the electrons loss during the transport process (Fig. 7). ${ }^{73,146}$ Shen et al. proposed a CdSe sensitized $\mathrm{TiO}_{2}-\mathrm{NT}$ working electrode and constructed a complete QDSC device, achieving a PCE of $1.8 \% .{ }^{122}$ Meng et al. fabricated a $\mathrm{CdS} / \mathrm{CdSe}$ sensitized $\mathrm{TiO}_{2}$-NT photoanode and obtained a PCE of $3.18 \%$ with the optimization of CdSe deposition time and the length of the nanotube. ${ }^{128}$ Zhang et al. applied short-length and high-density rutile $\mathrm{TiO}_{2}-\mathrm{NR}$ array as electrode and sensitized with $\mathrm{PbS}$ QDs to fabricate all solid-state QDSCs, delivering a PCE of $4.10 \% .{ }^{147}$ Kuang et al. employed vertically aligned anatase $\mathrm{TiO}_{2}-\mathrm{NW}$ with branched architecture as ETM in CdS/CdSe QDSCs (Fig. 8). The branched hierarchical structure was found to favor the improvement of $J_{\text {sc }}$ value due to the increased light-scattering ability and a final PCE of $4.2 \%$ was achieved. ${ }^{144}$ Furthermore, they designed a three-dimensional (3D) hierarchically branched $\mathrm{TiO}_{2}-\mathrm{NW}$ coated hollow sphere photoanode with high specific surface area while maintaining roomy space, further boosting the PCE to $6.01 \%{ }^{145}$ 

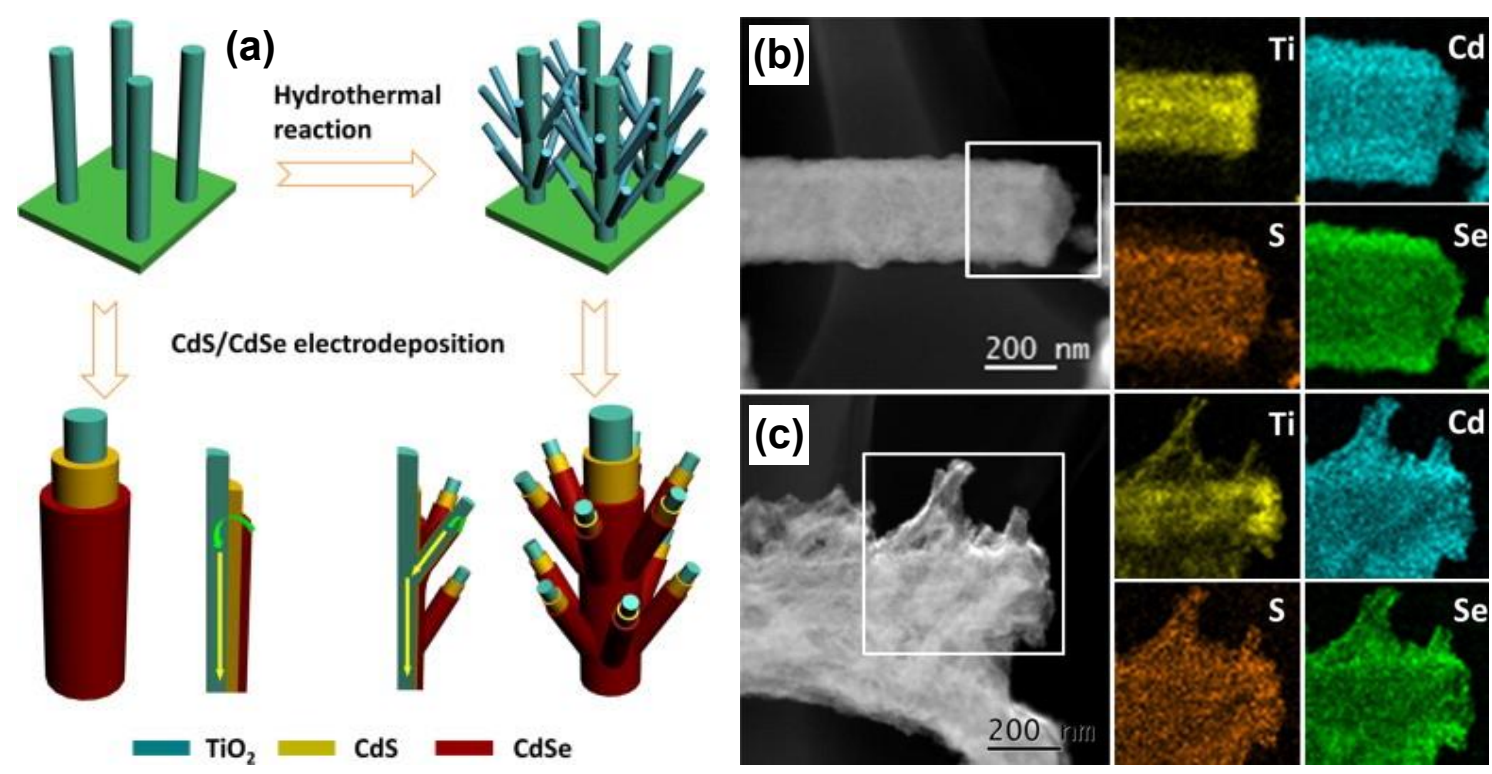

Fig. 8 (a) A schematic diagram showing the preparation process and structure of $\mathrm{CdS} / \mathrm{CdSe}$ co-sensitized smooth and hierarchical $\mathrm{TiO}_{2}$ nanowire, as well as the sketch path of electron injection (green arrow) and electron transport (yellow arrow). High-angle annular dark-field scanning transmission electron microscopy (HAADF-STEM) and energy dispersive X-Ray spectro-scopy (EDX) mappings of $\mathrm{TiO}_{2} / \mathrm{CdS} / \mathrm{CdSe}$ photoanode based on smooth (b) and hierarchical (c) nanowires. Reprinted with permission from ref. 144. Copyright (2014) Elsevier.

Although much efforts have been devoted to explore 1D structured $\mathrm{TiO}_{2}$ based photoanode in QDSCs, the obtained PCEs are still poor compared to that of the $\mathrm{TiO}_{2}-\mathrm{NP}$ based ones. Therefore, the potential of $1 \mathrm{D}$ structured $\mathrm{TiO}_{2}$ based photoanode has not been fully explored. This may be mainly derived from the low QDs loading amount on the $1 \mathrm{D}$ structured $\mathrm{TiO}_{2}$ film, resulting in low light harvesting efficiency and severe charge recombination. Therefore, further improvement of the performance of 1D structured $\mathrm{TiO}_{2}$ based QDSCs should rely on the enhancement of the specific surface area of the photoanode film and therefore increase the QD loading amount on $\mathrm{TiO}_{2}$ film. 
Table 2 Summary of the representative photovoltaic performance for QDSCs based on different ETMs.

\begin{tabular}{|c|c|c|c|c|c|c|c|c|}
\hline ETM & QDs & $\mathbf{C E}$ & electrolyte & $J_{\mathrm{sc}}\left(\mathrm{mA} \mathrm{cm}{ }^{-2}\right)$ & $V_{\mathrm{oc}}(\mathrm{V})$ & FF & PCE (\%) & Ref. \\
\hline $\mathrm{TiO}_{2}-\mathrm{NP}$ & ZCISe-CdSe & $\mathrm{MC} / \mathrm{Ti}$ & polysulfide & 27.39 & 0.752 & 0.619 & 12.75 & 112 \\
\hline $\mathrm{TiO}_{2}-\mathrm{NW}$ & $\mathrm{CdS} / \mathrm{CdSe}$ & $\mathrm{Cu}_{2} \mathrm{~S} / \mathrm{FTO}$ & polysulfide & 19.32 & 0.531 & 0.586 & 6.01 & 145 \\
\hline $\mathrm{TiO}_{2}-\mathrm{NT}$ & $\mathrm{PbS}$ & $\mathrm{Pt}$ & $\mathrm{I}^{-} / \mathrm{I}_{3}^{-}$ & 8.48 & 0.64 & 0.63 & 3.41 & 127 \\
\hline $\mathrm{TiO}_{2}-\mathrm{NR}$ & $\mathrm{PbS}$ & - & spiro-OMeTAD & 13.56 & 0.52 & 0.579 & 4.10 & 133 \\
\hline $\mathrm{TiO}_{2}-\mathrm{NS}$ & $\mathrm{CdSe}$ & $\mathrm{Cu}_{2} \mathrm{~S} /$ brass & polysulfide & 16.95 & 0.591 & 0.50 & 5.01 & 148 \\
\hline $\mathrm{ZnO}-\mathrm{NP}$ & $\mathrm{CdS} / \mathrm{CdSe}$ & $\mathrm{Cu}_{2} \mathrm{~S} /$ brass & polysulfide & 10.48 & 0.683 & 0.623 & 4.46 & 149 \\
\hline $\mathrm{ZnO}-\mathrm{NW}$ & $\mathrm{Zn}_{\mathrm{x}} \mathrm{Cd}_{1-\mathrm{x}} \mathrm{Se}$ & $\mathrm{Cu}_{2} \mathrm{~S} /$ brass & polysulfide & 18.05 & 0.65 & 0.40 & 4.74 & 105 \\
\hline $\mathrm{ZnO}-\mathrm{NW}$ & $\mathrm{ZnSe} / \mathrm{CdSe}$ & $\mathrm{Cu}_{2} \mathrm{~S} /$ brass & polysulfide & 11.96 & 0.836 & 0.45 & 4.54 & 150 \\
\hline $\mathrm{ZnO}-\mathrm{NR}$ & $\mathrm{CdS} / \mathrm{CdSe}$ & $\mathrm{Cu}_{2} \mathrm{~S} /$ brass & polysulfide & 13.28 & 0.642 & 0.567 & 4.83 & 151 \\
\hline $\mathrm{ZnO}-\mathrm{TP}$ & $\mathrm{ZnSe} / \mathrm{CdSe} / \mathrm{ZnSe}$ & $\mathrm{Cu}_{2} \mathrm{~S}-\mathrm{RGO}$ & polysulfide & 17.3 & 0.761 & 0.471 & 6.2 & 152 \\
\hline $\mathrm{SnO}_{2}$ & $\mathrm{CdS} / \mathrm{CdSe}$ & $\mathrm{Cu}_{2} \mathrm{~S}$ & polysulfide & 10.13 & 0.700 & 0.616 & 4.37 & 153 \\
\hline $\mathrm{NiO}$ & $\mathrm{PbS}$ & $\mathrm{Pt}$ & {$\left[\mathrm{Co}(\mathrm{dtb}-\mathrm{bpy})_{3}\right]^{2+/ 3+}$} & 5.27 & 0.227 & 0.33 & 0.40 & 154 \\
\hline
\end{tabular}

4.1.2. $\mathrm{ZnO}$ based photoanode. From the aspect of electronic feature Compared to $\mathrm{TiO}_{z}$, $\mathrm{ZnO}$ seems to be more suitable in serving as ETM due to its higher electron mobility (130-200 vs 0.1-4 $\mathrm{cm}^{2} \mathrm{~V}^{-1} \mathrm{~s}^{-1}$ ) and conduction band edge. These unique properties are beneficial for obtaining higher $V_{\text {oc }}$ values. ${ }^{42,47}$ Hence, much work about $\mathrm{ZnO}$ based photoanode has been reported. However, from the aspect of chemical stability, $\mathrm{ZnO}$ electrode is limited with lower stability than $\mathrm{TiO}_{2}$. The amphoteric nature renders $\mathrm{ZnO}$ dissolves in both acidic and basic media and therefore limits its application in QDSCs. The chemical instability would limit the long-term lifetime of cell devices. Another restriction is that its pre-functionalisation is not possible since dissolution of $\mathrm{ZnO}$ by the acidic carboxylic anchoring groups of the capping ligands on QD sensitizers.

Similar to $\mathrm{TiO}_{2}, \mathrm{ZnO}$ nanoparticle $(\mathrm{ZnO}-\mathrm{NP})$ based photoanode was also investigated in QDSCs. ${ }^{149,155-162}$ For example, Stathatos et al. prepared a ZnO-NP (10-30 nm) based film through a facile method with use of an amino double edged polypropylene oligomer and zinc precursor. ${ }^{160}$ The film was used to fabricate quasi-solid-state CdS/CdSe sensitized QDSCs, 
delivering a PCE of $4.5 \%$. Meng et al. prepared ZnO-NPs with diameter of $20 \mathrm{~nm}$ based film and then sensitized with $\mathrm{CdS} / \mathrm{CdSe}$ QDs to construct photoanode, demonstrating a PCE of $4.46 \%{ }^{149}$ Cao and Tian et al. applied a passivation strategy on the surface of $\mathrm{ZnO}$ nanoparticles with use of $\mathrm{TiO}_{2}$ nanoparticles to reduce the charge recombination at the photoanode/electrolyte interface. ${ }^{161}$ The results demonstrated that the $\mathrm{TiO}_{2}$ passivation can not only suppress the charge recombination process, but also increased the specific surface area for more QDs loading, improving the PCE of CdS/CdSe based QDSCs from $2.38 \%$ to 4.68\%. They also reported a bilayer structured photoanode with $\mathrm{ZnO}-\mathrm{NP}$ film as transparent layer and $\mathrm{ZnO}$ microsphere (MS) as scattering layer for CdS/CdSe based QDSCs. ${ }^{162}$ The MS layer could effectively increase the light diffuse reflection so as to enhance the light harvesting efficiency, exhibiting a PCE of 5.08\%. Nevertheless, unlike the case of $\mathrm{TiO}_{2}$, the solar cell performance of ZnO-NP based QDSCs were inferior to that of the multi-dimensional $\mathrm{ZnO}$ based ones, partially due to the severe charge recombination losses at the $\mathrm{ZnO} /$ electrolyte interface.

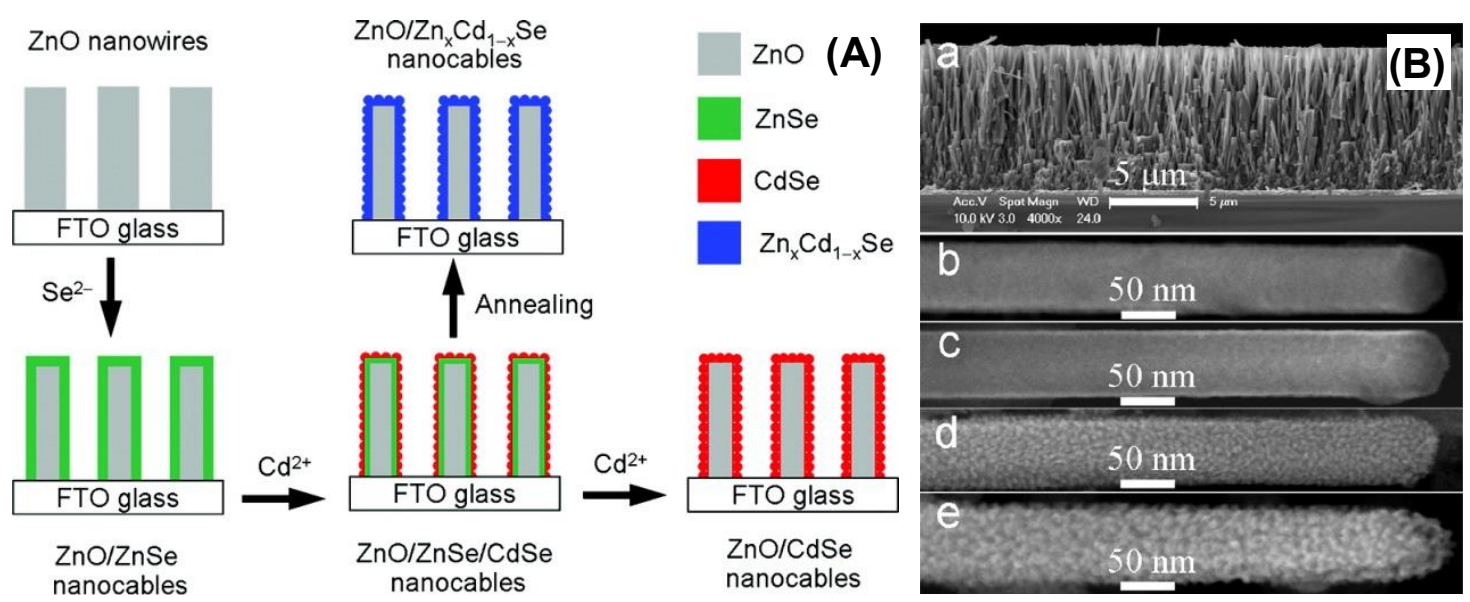

Fig. 9 (A) Schematic illustration for the formation processes of the $\mathrm{ZnO} / \mathrm{Zn}_{\mathrm{x}} \mathrm{Cd}_{1-\mathrm{x}} \mathrm{Se}$ core/shell nanocables. (B) SEM images of (a) a $\mathrm{ZnO}$ nanowire array, (b) a $\mathrm{ZnO} / \mathrm{ZnSe}$ nanocable, (c) a $\mathrm{Zn}_{0.7} \mathrm{Cd}_{0.3}$ Se nanocable prepared by reacting a $\mathrm{ZnO} / \mathrm{ZnSe}$ nanocable with $\mathrm{Cd}^{2+}$ at $50{ }^{\circ} \mathrm{C}$, (d) a $\mathrm{ZnO} / \mathrm{Zn}_{0.33} \mathrm{Cd}_{0.67} \mathrm{Se}$ nanocable prepared by reacting a $\mathrm{ZnO} / \mathrm{ZnSe}$ nanocable with $\mathrm{Cd}^{2+}$ at $90{ }^{\circ} \mathrm{C}$, and (e) a $\mathrm{ZnO} / \mathrm{CdSe}$ nanocable prepared by reacting a $\mathrm{ZnO} / \mathrm{ZnSe}$ nanocable with $\mathrm{Cd}^{2+}$ at $140{ }^{\circ} \mathrm{C}$. Reprinted with permission from ref. 105. Copyright (2011) American Chemical Society.

Another unique property of $\mathrm{ZnO}$ is that it is relatively easy to crystallize and the growth is anisotropic, so that $\mathrm{ZnO}$ nanostructures in various morphologies can be easily prepared. ${ }^{47}$ 
Accordingly, $\mathrm{ZnO}$ nanowire $(\mathrm{ZnO}-\mathrm{NW}),{ }^{105,150,163-175}$ nanorod $(\mathrm{ZnO}-\mathrm{NR}),{ }^{176-186}$ and nano-tetrapod $(\mathrm{ZnO}-\mathrm{TP})^{187,188}$ have been employed as ETM to construct photoanodes in QDSCs. For example, in 2007, Norris and Aydil et al. grew ZnO-NW on FTO substrate and sensitized with CdSe QDs. ${ }^{163}$ They fabricated QDSCs using $\mathrm{I}^{-} / \mathrm{I}_{3}{ }^{-}$as electrolyte and Pt as CE, delivering a PCE of $0.4 \%$. After that, Yong et al. sensitized $\mathrm{ZnO}-\mathrm{NW}$ with $\mathrm{CdS} / \mathrm{CdSe}$ QDs through SILAR approach, improving the PCE of ZnO-NW based QDSCs to $4.15 \% .{ }^{175}$ Due to the much larger $K_{\mathrm{sp}}$ value of $\mathrm{Zn}(\mathrm{OH})_{2}, \mathrm{ZnO}$ can be easily converted to $\mathrm{ZnS}$ or $\mathrm{ZnSe}$. This property can be used to form QDs sensitizers around $\mathrm{ZnO}$ through a facile ion exchange approach. For example, Lee et al. prepared ZnO-NW arrays on FTO substrate, and then formed a $\mathrm{ZnO} / \mathrm{ZnSe} / \mathrm{CdSe}$ nanocable structured photoanode through an ion exchange approach. ${ }^{150}$ The QDSCs based on this nanocable photoanode exhibited a PCE of $4.54 \%$ and a $V_{\text {oc }}$ as high as $0.836 \mathrm{~V}$. The ZnSe layer was found to be crucial in achieving high efficiency since it can reduce charge recombination by passivating the surface of $\mathrm{ZnO}-\mathrm{NW}$ and up-shifts the $\mathrm{CB}$ of $\mathrm{ZnO}$. Furthermore, employing the same deposition method, they fabricated a $\mathrm{ZnO} / \mathrm{Zn}_{\mathrm{x}} \mathrm{Cd}_{1-\mathrm{x}} \mathrm{Se}$ core/shell structured nanocables photoanode (Fig. 9). ${ }^{105}$ The band gap of the nanocables can be tuned by the $\mathrm{Zn}$ content in the $\mathrm{Zn}_{\mathrm{x}} \mathrm{Cd}_{1-\mathrm{x}} \mathrm{Se}$ shell layer. Finally, a PCE of 4.74\% was obtained with use of this nanocable as photoanode. It is well known that a key problem for the $\mathrm{ZnO}$ ETM is that the charge recombination at the interface of $\mathrm{ZnO}$ /electrolyte is more serious than that of $\mathrm{TiO}_{2}$ based system, leading to the poor cell efficiency. While, it was found that the treatment of $\mathrm{ZnO}$ surface with use of $\mathrm{TiO}_{2}$ is an efficient way to inhibit interface charge recombination. For example, Cao and Tian et al. found that after the modification of the $\mathrm{ZnO}-\mathrm{NR}$ arrays with $\mathrm{TiO}_{2}$ nanoparticles, the PCE of the cell can be improved from $1.54 \%$ to $3.14 \% .{ }^{181} \mathrm{The}^{\mathrm{TiO}_{2}}$ nanoparticles can not only act as a barrier layer to prevent the electron back transfer from $\mathrm{ZnO}$ to electrolyte, but also altered the surface characteristics of the $\mathrm{ZnO}-\mathrm{NR}$ so as to improve QDs loading amount. 


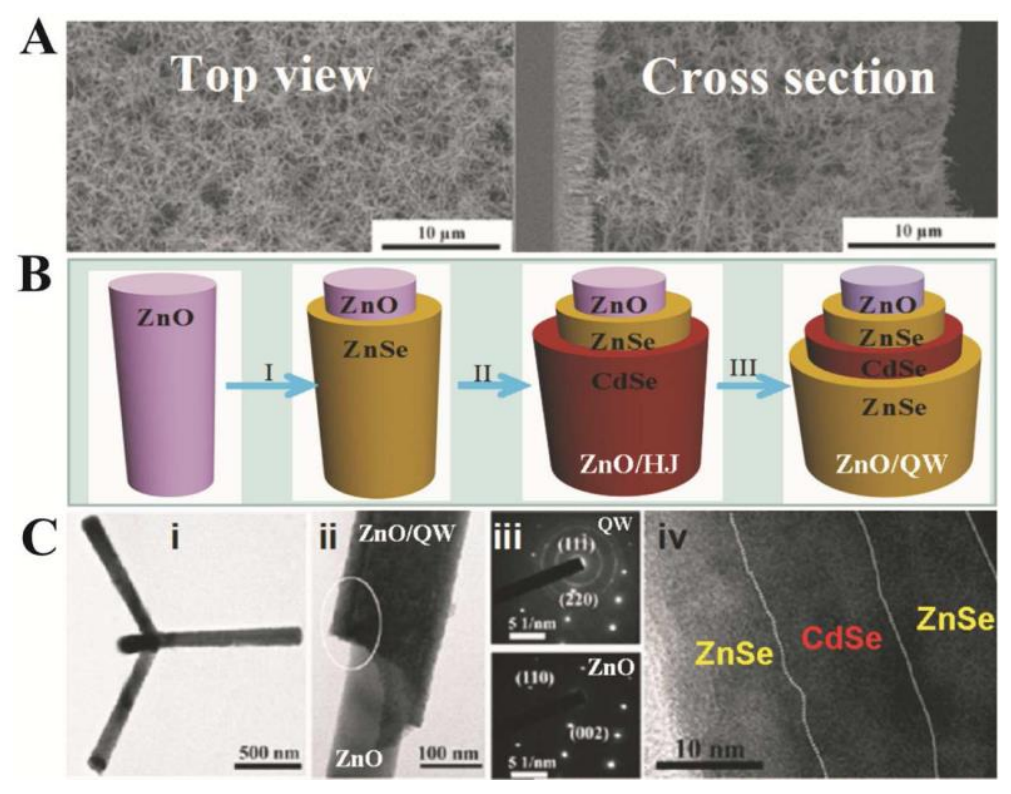

Fig. 10 Layer by layer formation of the $\mathrm{ZnSe} / \mathrm{CdSe} / \mathrm{ZnSe}$ quantum well (QW) sensitizer. (A) Top and cross-sectional view SEM images of $\mathrm{ZnO}$ nanotetrapods photoanode for SSSCs. (B) Schematic diagram of the formation process: (i) Place the photoanode in freshly prepared NaHSe; (ii) dip in $\mathrm{Cd}^{2+}$ and NaHSe solution successively for four cycles; (iii) dip in $\mathrm{Zn}^{2+}$ and NaHSe solution successively for two cycles. (C) TEM images of QW-sensitized tetrapod, the arm apart from tetrapod and electron diffractions corresponding to different sections with and without QW shell and structure. Reprinted with permission from ref. 188. Copyright (2013) American Chemical Society.

A key limitation for the $\mathrm{ZnO}-\mathrm{NW}$ and $\mathrm{ZnO}-\mathrm{NR}$ based photoanode is the low specific surface area, leading to the low QD loading amount and therefore resulting in a low $J_{\text {sc }}$ value. Thus, hierarchical architecture comprising long nanowires trunks and short nanorod branches is a suitable choice to offer more sites for QDs loading. Yang et al. reported a $\mathrm{ZnO}$ double-layer architecture based photoanode for QDSCs, consisting of a ZnO-NR underlayer and a $\mathrm{ZnO}$-TP top layer. ${ }^{187}$ It was shown that the double-layer strategy could reduce charge recombination at the interface between FTO substrate and $\mathrm{ZnO}$, yielding a higher $V_{\mathrm{oc}}$ of 0.703 $\mathrm{V}$ and a PCE of 5.24\%. Furthermore, Yang and coworkers prepared ZnO-TP with diameters of 50-200 nm and lengths of 400-1000 nm, followed by sensitization with $\mathrm{ZnSe} / \mathrm{CdSe} / \mathrm{ZnSe}$ quantum wells (QWs) (Fig. 10). ${ }^{188}$ The QWs sensitizers based QDSCs delivered a record PCE of $6.20 \%$ and an impressive high $V_{\text {oc }}$ of $0.761 \mathrm{~V}$, which is higher than the $\mathrm{ZnSe} / \mathrm{CdSe}$ heterojunction QDs based one (4.02\%). A core/shell two-channel transport mechanism was 
proposed and demonstrated for the QWs based solar cells, indicating that the electron could transport through both the $\mathrm{ZnO}$ and sensitizer channel. Up to now, this is the highest PCE for the ZnO ETM based QDSCs.

On the whole, the PCEs of ZnO ETM based QDSCs still lag far behind that of $\mathrm{TiO}_{2} \mathrm{ETM}$ based one. Nevertheless, ZnO ETM owns great potential to realize high efficiency QDSCs due to its unique properties, especially its high electron mobility. It should be noted that the obtained $V_{\text {oc }}$ of $\mathrm{ZnO}$ based QDSCs is obviously higher than that of the $\mathrm{TiO}_{2}$ based one. So far, the highest $V_{\mathrm{oc}}$ for $\mathrm{ZnO}$ based QDSCs is as high as $0.836 \mathrm{~V}$, which is about $0.2 \mathrm{~V}$ higher than that of the $\mathrm{TiO}_{2}$ based one. ${ }^{150}$ Thus, it is necessary and meaningful to further improve the PCE of $\mathrm{ZnO}$ based QDSCs through optimizing the QD sensitizers and interface characteristics.

4.1.3. Other kinds of ETM. Apart from $\mathrm{TiO}_{2}$ and $\mathrm{ZnO}$, other kinds of metal oxide, such as $\mathrm{SnO}_{2},{ }^{153,189} \mathrm{ZrO}_{2},{ }^{190} \mathrm{NiO}$, and $\mathrm{Zn}_{2} \mathrm{SnO}_{4}{ }^{191}$ et al. have also been explored as ETM in QDSCs. However, the cell performance based on these ETMs was usually poor, partially due to the unsatisfactory band edge position or the low electron mobility of the corresponding ETM. Among these, Meng et al. prepared highly ordered $\mathrm{SnO}_{2}$ inverse opal films with different thicknesses as photoanode in CdS/CdSe based QDSCs, delivering a PCE of $4.37 \% .{ }^{153}$ Zaban et al. demonstrated that the electron injection process can also take place in $\mathrm{ZrO}_{2}$ based QDSCs.

4.1.4. p-Type metal oxide semiconductors and p-type QDSCs. The above mentioned wide band gap semiconductors (such as $\mathrm{TiO}_{2}$ and $\mathrm{ZnO}$ ), are of n-type, which are responsible for extracting photogenerated electron from QD sensitizers in a QDSC. Another type of metal oxide semiconductors are of p-type, which take the role of extracting photogenerated hole from QD sensitizers in a QDSC. A classic example of p-type semiconductor is $\mathrm{NiO}$, which is widely used for photoelectronic devices. Copper(I) thiocyanate, $\mathrm{CuSCN}$, is a promising alternative to $\mathrm{NiO}$ as an inorganic p-type semiconductor material. Correspondingly, QDSCs based on the sensitization of a n-type semiconductor oxide like $\mathrm{TiO}_{2}$ or $\mathrm{ZnO}$ are known as standard (Grätzel) cells, and the cells based on the sensitization of a wide band gap p-type semiconductor oxides such as $\mathrm{NiO}$ are referred to p-type (inverted) QDSCs. Usually, unless specially specified, QDSCs are referred to standard ones. The extraction rate of a hole from QD to redox couple will be inherently slower than that of an electron to n-type semiconductor matrix. ${ }^{39,70,192}$ This leads to unbalanced charge transport in the standard QDSCs, and p-type 
QDSCs have the potential to solve this problem. Photophysical charge transfer measurement results indicated that hole extraction rates in p-type QDSCs are in the same range as electron injection rates in conventional n-type QDSCs. ${ }^{193-195}$ Moreover, the most promising application of p-type sensitized solar cells is the construction of a tandem configuration with the combination of a p-type and an n-type one to overcome the Shockley-Queisser limit. ${ }^{196}$

Even though the concept of p-type QDSCs is very appealing, the efficiencies for most of them are very low or not reported, ${ }^{197-199}$ with one exception of $1.25 \%$ reported by Aldakov and coworkers in 2016. ${ }^{193} \mathrm{Up}$ to date, almost all p-type QDSCs are based on $\mathrm{NiO}$ photocathodes due to the scarcity of transparent p-type nanostructured semiconductors. Recently CuSCN has been proven to be a promising alternative to $\mathrm{NiO}$ as a p-type material scaffold in liquid-junction QDSCs. ${ }^{194}$ In this report, CuSCN nanowires were used as photocathode in the construction of p-QDSSCs, and efficient sensitization by $\mathrm{CuInS}_{\mathrm{x}} \mathrm{Se}_{2-\mathrm{x}}$ QDs was observed.

\subsection{QDs sensitizers}

QD is the core component in QDSCs that performs the function of harvesting sunlight and then generating electron-hole pairs. ${ }^{24,26,30}$ Ideal QDs sensitizers should possess the following characteristics: (1) higher conduction band edge relative to that of the ETM for effective electron injection; (2) narrow band gap to absorb sunlight over a wide range of solar spectrum and high absorption coefficient for high light harvesting efficiency; (3) good stability towards light, heat, and electrolyte; (4) simple preparation and low toxicity. Apart from the design of QD structure, the QD deposition route on metal oxide substrate is also determinable to the final photovoltaic performance of the solar cells. Herein, we will discuss the different methods to deposit QD on metal oxide substrate and the various QD sensitizers that have been explored in QDSCs. The representative photovoltaic performances for QDSCs based on different QDs are summarized in Table 3.

4.2.1. QD deposition routes. Unlike the molecular dye, QDs are larger sized inorganic nanoparticles. It is difficult to tether the QD onto metal oxide to form a fully-covered monolayer due to the lack of anchoring site on inorganic particles. Therefore, it is challenging to obtain a QD sensitized photoanode with high QD loading amount. ${ }^{36,44,200}$ Typically, the QD deposition techniques can be divided into two kinds: in situ and ex situ approaches. As shown in Fig. 11, for the in situ deposition, QDs are grown directly on metal oxide substrate from ionic precursor solutions, including chemical bath deposition (CBD) and successive ionic 
layer adsorption and reaction (SILAR) approaches. For the ex situ approach, colloidal QDs are pre-synthesized and then deposited onto metal oxide substrate through direct adsorption, electrophoretic deposition, or linker-assisted deposition methods.

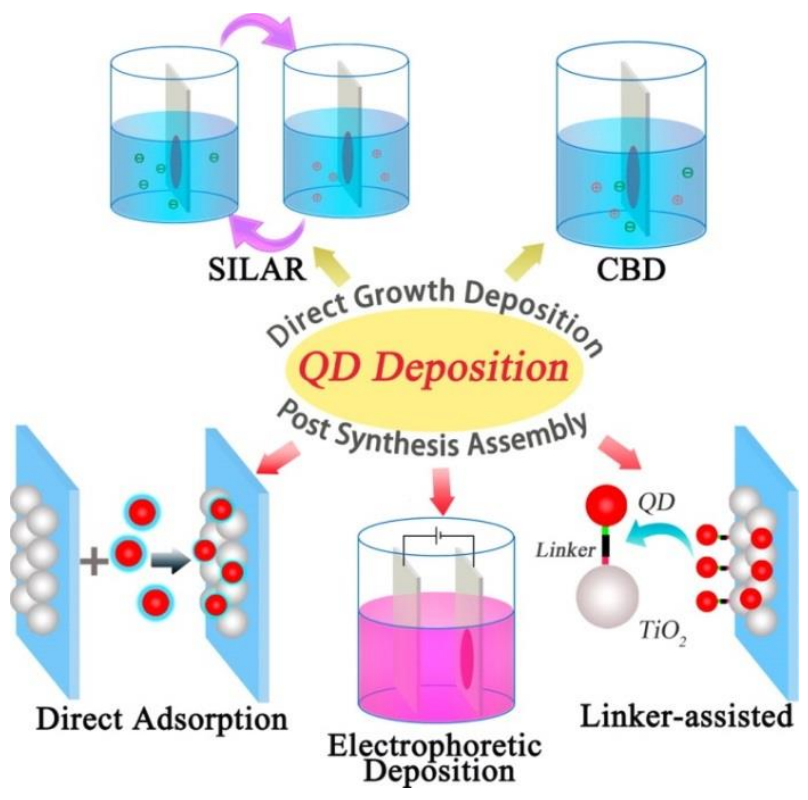

Fig. 11 Schematic illustration of QD deposition approaches. Direct growth method (above): (i) SILAR and (ii) CBD. Postsynthesis assembly deposition method (below): (i) direct adsorption, (ii) electrophoretic deposition (EPD), and (iii) linker-assisted assembly. Reprinted with permission from ref. 36. Copyright (2015) American Chemical Society.

Due to the distinct advantages of easy processability, good reproducibility and high QD loading amount, until now, the in situ deposition method is widely adopted to assemble QDSCs. In the CBD growth route, a bath solution containing cationic and anionic precursors is prepared. ${ }^{97,201-203}$ When immersing the metal oxide film in the bath solution, QDs can grow directly on the surface of the metal oxide. The growth of QD can be controlled by varying the immersing time of the film in the solution as well as the reaction temperature. For the SILAR deposition, cationic and anionic precursors are separated and the metal oxide film is dipped into the two solutions alternatively. ${ }^{101}$ The growth of QD is mainly controlled by the immersing cycles. Although a high QDs loading amount can be achieved by the in situ deposition method, it is difficult to control the QD size and size distribution as well as the density of trap state defects. As a result, QDs obtained through this route usually have high density of trap states, resulting in severe charge recombination. Therefore, the highest PCE of QDSCs deriving from the in situ QD deposition method is only about 7\%. ${ }^{192,203}$ 
Table 3 Summary of the representative photovoltaic performance for QDSCs based on different QD sensitizers.

\begin{tabular}{|c|c|c|c|c|c|c|c|}
\hline QDs & $\mathbf{C E}$ & electrolyte & $J_{\mathrm{sc}}\left(\mathrm{mA} \mathrm{cm} \mathrm{cm}^{-2}\right)$ & $V_{\mathrm{oc}}(\mathbf{V})$ & FF & PCE (\%) & Ref. \\
\hline $\mathrm{CdS}$ & $\mathrm{C}$-fabric/ $\mathrm{WO}_{3-\mathrm{x}}$ & polysulfide & 10.38 & 0.891 & 0.64 & 5.91 & 204 \\
\hline $\mathrm{CdSe}$ & $\mathrm{Cu}_{2} \mathrm{~S} /$ brass & polysulfide & 16.01 & 0.693 & 0.68 & 7.54 & 205 \\
\hline $\mathrm{CdTe}$ & $\mathrm{Cu}_{2} \mathrm{~S} /$ brass & polysulfide & 16.58 & 0.629 & 0.694 & 7.24 & 206 \\
\hline $\mathrm{PbS}$ & $\mathrm{Cu}_{2} \mathrm{~S} /$ brass & polysulfide & 29.98 & 0.398 & 0.468 & 5.58 & 207 \\
\hline $\mathrm{Sb}_{2} \mathrm{~S}_{3}$ & $\mathrm{Au}$ & PEDOT/PCPDTBT & 16.1 & 0.711 & 0.65 & 7.5 & 208 \\
\hline InP & $\mathrm{Cu}_{2} \mathrm{~S} /$ brass & polysulfide & 10.58 & 0.59 & 0.567 & 3.54 & 209 \\
\hline $\mathrm{Ag}_{2} \mathrm{~S}$ & $\mathrm{Pt}$ & polysulfide & 9.28 & 0.509 & 0.52 & 2.41 & 210 \\
\hline $\mathrm{Au}_{\mathrm{x}}$ & $\mathrm{Pt}$ & {$\left[\mathrm{Co}(\mathrm{bpy})_{3}\right]^{2+/ 3+}$} & 3.96 & 0.832 & 0.716 & 2.36 & 211 \\
\hline carbon dot & $\mathrm{Cu}_{2} \mathrm{~S} /$ brass & polysulfide & 6.47 & 0.43 & 0.31 & 0.87 & 212 \\
\hline $\mathrm{PbS} / \mathrm{CdS}$ & $\mathrm{Cu}_{2} \mathrm{~S} / \mathrm{FTO}$ & polysulfide & 18.81 & 0.595 & 0.642 & 7.19 & 213 \\
\hline $\mathrm{CdS} / \mathrm{CdSe}$ & $\mathrm{Cu}_{2} \mathrm{~S} /$ brass & polysulfide & 21.49 & 0.61 & 0.55 & 7.24 & 214 \\
\hline $\mathrm{CdTe} / \mathrm{CdSe}$ & $\mathrm{Cu}_{2} \mathrm{~S} /$ brass & polysulfide & 19.59 & 0.606 & 0.569 & 6.76 & 74 \\
\hline $\mathrm{ZnTe} / \mathrm{CdSe}$ & $\mathrm{Cu}_{2} \mathrm{~S} /$ brass & polysulfide & 19.35 & 0.646 & 0.55 & 6.89 & 85 \\
\hline $\mathrm{CdTe} / \mathrm{CdS}$ & $\mathrm{Au}$ & polysulfide & 13.60 & 0.682 & 0.41 & 3.80 & 215 \\
\hline $\mathrm{CdTe} / \mathrm{CdS} / \mathrm{CdS}$ & $\mathrm{Cu}_{2} \mathrm{~S} /$ brass & polysulfide & 20.19 & 0.61 & 0.51 & 6.32 & 216 \\
\hline $\mathrm{ZnSe} / \mathrm{CdS}$ & $\mathrm{Pt}$ & polysulfide & 2.29 & 0.44 & 0.27 & 0.27 & 217 \\
\hline $\mathrm{ZnSe} / \mathrm{CdSe}$ & $\mathrm{Cu}_{2} \mathrm{~S} /$ brass & polysulfide & 11.96 & 0.836 & 0.45 & 4.54 & 150 \\
\hline $\mathrm{ZnSe} / \mathrm{CdSe} / \mathrm{ZnSe}$ & $\mathrm{Cu}_{2} \mathrm{~S}-\mathrm{RGO}$ & polysulfide & 17.3 & 0.761 & 0.471 & 6.2 & 152 \\
\hline $\mathrm{CdSe}_{\mathrm{x}} \mathrm{S}_{1-\mathrm{x}}$ & $\mathrm{Cu}_{2} \mathrm{~S}-\mathrm{RGO}$ & polysulfide & 11.2 & 0.557 & 0.51 & 3.20 & 218 \\
\hline $\mathrm{CdSe}_{\mathrm{x}} \mathrm{Te}_{1-\mathrm{x}}$ & $\mathrm{MC} / \mathrm{Ti}$ & polysulfide & 20.69 & 0.807 & 0.689 & 11.51 & 118 \\
\hline $\mathrm{Zn}_{\mathrm{x}} \mathrm{Cd}_{1-\mathrm{x}} \mathrm{Se}$ & $\mathrm{Cu}_{2} \mathrm{~S} /$ brass & polysulfide & 18.05 & 0.65 & 0.40 & 4.74 & 105 \\
\hline $\mathrm{CuInS}_{2}$ & $\mathrm{Cu}_{2} \mathrm{~S} / \mathrm{FTO}$ & polysulfide & 22.82 & 0.601 & 0.62 & 8.54 & 219 \\
\hline $\mathrm{Zn}-\mathrm{Cu}-\mathrm{In}-\mathrm{S}$ & $\mathrm{Cu}_{2} \mathrm{~S} /$ brass & polysulfide & 22.70 & 0.612 & 0.615 & 8.55 & 220 \\
\hline $\mathrm{CuInSe}_{2}$ & $\mathrm{Cu}_{2} \mathrm{~S} /$ brass & polysulfide & 26.93 & 0.528 & 0.57 & 8.10 & 221 \\
\hline $\mathrm{Zn}-\mathrm{Cu}-\mathrm{In}-\mathrm{Se}$ & $\mathrm{MC} / \mathrm{Ti}$ & polysulfide & 25.97 & 0.752 & 0.644 & 12.57 & 222 \\
\hline $\mathrm{Cu}-\mathrm{In}-\mathrm{Ga}-\mathrm{Se}$ & $\mathrm{MC} / \mathrm{Ti}$ & polysulfide & 25.01 & 0.740 & 0.621 & 11.49 & 223 \\
\hline
\end{tabular}




\begin{tabular}{llllllll}
$\mathrm{CuInSe}_{x} \mathrm{~S}_{2-\mathrm{x}}$ & $\mathrm{Cu}_{\mathrm{x}} \mathrm{S} / \mathrm{FTO}$ & polysulfide & 16.8 & 0.56 & 0.59 & 5.51 & 78 \\
$\mathrm{Cu}_{2} \mathrm{ZnSnS}_{4}$ & $\mathrm{Cu}_{2} \mathrm{~S} /$ brass & polysulfide & 17.48 & 0.47 & 0.40 & 3.29 & 224 \\
\hline
\end{tabular}

Benefiting from the well-developed QD synthesis technique, high quality QDs can be facilely prepared through organometallic high temperature synthetic methods. ${ }^{225-227}$ There is no doubt that high quality QDs possess great potential for obtaining high efficiency QDSCs. The challenge for the use of pre-synthesized QDs in QDSCs is how to deposit them onto metal oxide film with high loading amount. The common methods to immobilize pre-synthesized QDs on metal oxide substrate include (i) direct adsorption, ${ }^{95,96,228-231}$ (ii) electrophoretic deposition (EPD), ${ }^{232,233}$ and (iii) linker molecule-assisted self-assembly. ${ }^{36,44,163,228,234,235}$ In the initial stage, direct adsorption and EPD methods were commonly used to bind pre-synthesized QDs on metal oxide film. Whereas, the obtained PCEs are usually low, mainly due to the low QDs loading amount and therefore shadowing the superiority of the high quality nature of the pre-synthesized QDs.

As it has been already commented, the capping ligand induced self-assembly (CLIS)

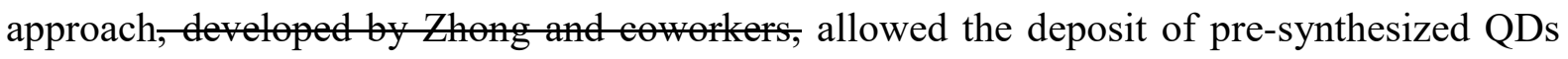
on $\mathrm{TiO}_{2}$ electrode with a high loading amount. . $^{36,74,85,111,113-119}$ High quality QDs were first prepared in organic phase, followed with a ligand exchange process to obtain linker molecule capped water-soluble QDs, which can be then effectively deposited on $\mathrm{TiO}_{2}$ film electrodes. As early as 1990s, Alivisatos et al. immobilized CdS and CdSe QDs on gold or aluminum surfaces in virtue of bifunctional group molecules containing thiol and carboxylate groups. ${ }^{236}$ Later work borrowed this technique to assemble QDs on $\mathrm{TiO}_{2}$ film electrode in the fabrication of QDSCs. ${ }^{10,163,230,237-240}$

Despite the advantages of the CLIS approach, researchers still failed to achieve high loading amount of QD sensitizers on $\mathrm{TiO}_{2}$ electrode, and high photovoltaic performance of the resulting QDSCs. Through improving ligand exchange techniques as well as optimizing QD deposition conditions, in 2012 Zhong's group exploited this deposition approach into an practicable and effective one to realize fast and high loading amount of QDs on $\mathrm{TiO}_{2}$ films. ${ }^{109,110}$ Some special factors, including the choice of capping ligand, excess linker molecule in the final QD solution, and the $\mathrm{pH}$ of the QD solution were found to be crucial to realize effective QD deposition. This novel deposition method was proven to be capable of realizing fast, uniform and high loading amount of $\mathrm{QDs}$ on $\mathrm{TiO}_{2}$ electrode (Fig. 12). Since 
then, the record PCE of QDSCs has been rapidly improving to nearly $13 \%$ now. ${ }^{112}$

Although the CLIS deposition approach is very effective in the fabrication of high efficiency QDSC, this process involves a tedious additional step of ligand exchange with use of a short-chain mercapto-alkylcarboxyl ligand as phase transfer reagent to displace the initial long carbon chain ligands around QDs prepared via an organometallic high temperature route. The synthesis of colloidal QDs capped with a short-chain mercapto-alkylcarboxyl ligand directly in aqueous media (denoted as aqueous QDs henceforth) provides a straightforward access to immobilize QD sensitizer onto the $\mathrm{TiO}_{2}$ surface in photoanodes. This approach obviates the additional step for ligand exchange, thereby simplifying the QD sensitization process. Attempts of using aqueous QDs in the fabrication of QDSCs can be dated as early as $2009,{ }^{241}$ but no photovoltaic performance was reported. In 2011, Meng and coworker employed aqueous $\mathrm{CuInS}_{2}$ in the fabrication of QDSCs with PCE of $1.47 \%{ }^{242}$ Following this, a series of higher PCEs have been obtained through optimizing the deposition conditions (such as $\mathrm{pH}$ values of QD solution, introduction of free ligand), tuning the electronic structure of QD sensitizers etc. ${ }^{198,211,216,243-248} \mathrm{Up}$ to date, the reported highest PCE with use of aqueous QD sensitizer under the irradiation of one full sun light is $8.0 \%$, and a PCE of $8.15 \%$ was also obtained but under light intensity of $30 \mathrm{~mW} \mathrm{~cm}^{-2} \cdot{ }^{249,250}$ It is noted that the aqueous QD sensitizers were mostly concentrated on $\mathrm{CuInS}_{2}$, and CdTe based QDs due to the relatively matured aqueous synthesis methods for these two kinds of QDs.
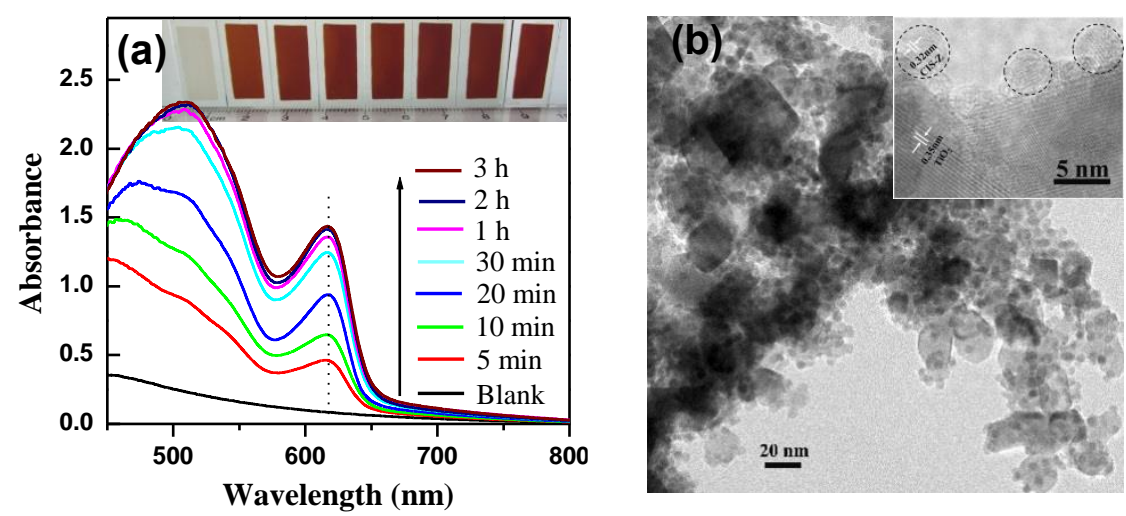

Fig. 12 (a) Temporal evolution of absorption spectra of CdSe QD sensitized $\mathrm{TiO}_{2}$ film. Inset: photographs of the films with increase of deposition time in turn. Reprinted with permission from ref. 110. Copyright (2012) Royal Society of Chemistry. (b) TEM images of $\mathrm{CuInS}_{2} / \mathrm{ZnS}$ QD (the small particles, $\sim 5 \mathrm{~nm}$ ) sensitized $\mathrm{TiO}_{2}$ film (the large particles $\sim 20 \mathrm{~nm}$ ). Inset: HRTEM micrograph. Reprinted with permission from ref. 116. Copyright (2014) American Chemical Society. 
4.2.2. Binary QDs. The binary QDs were widely used as sensitizers in the initial stage of QDSCs due to their mature synthesis recipe and the well-known photo-electronic properties. A variety of binary QDs, such as $\mathrm{InP},{ }^{95,209} \mathrm{InAs},{ }^{96} \mathrm{CdS},{ }^{146,202,251,252} \mathrm{CdSe},{ }^{99,100,110,200,203}$ $\mathrm{CdTe},{ }^{206,253} \mathrm{PbS},{ }^{127,207,213,254-259} \mathrm{Ag}_{2} \mathrm{~S},{ }^{169,210,260-262}$ etc. were applied as sensitizers in QDSCs. Among these, $\mathrm{CdS}$ and $\mathrm{CdSe}$ QDs are the most popular choice due to their simple preparation. Another distinct advantage of the binary QDs is that they can be directly grown on metal oxide substrate through the corresponding anion and cation in solution at low temperature, enabling a convenient route to prepare the photoanode. However, the problem of binary QDs is that it's difficult to well balance the narrower band gap and higher conduction band edge. For example, the conduction band edge of $\mathrm{CdS}$ and CdSe is suitable for charge separation, whereas their band gap is relatively wide so that the light harvesting range is narrow. On the contrary, $\mathrm{PbS}$ or $\mathrm{PbSe}$ QDs possess narrower band gap, but the conduction band edge is low and the electron injection efficiency is compromised. Therefore, the development of QDs sensitizer has turned to explore composite QDs to balance the light harvesting efficiency and electron injection efficiency.

4.2.3. Core/shell QDs. Since the first report of the wet chemical synthesis of core/shell QDs by Hines et al. in 1996, core/shell structured QDs have attracted much attention due to their unique properties. ${ }^{263-271}$ This core/shell strategy has also been shown to be effective in enhancing the chemical, thermal, and photochemical stability because the inorganic shell can provide a more robust protection to core QDs with respect to typical organic capping ligands. ${ }^{268-271}$ What's more, in core/shell QDs, the band edge alignment of the core and shell QDs enable us to tune the light absorption range, charge separation, and the recombination processes in QDSCs.

In QDSCs, core/shell QDs were first used as sensitizer by Lee et al. in $2009 .{ }^{101}$ In that work, authors deposited CdS/CdSe core/shell QDs through SILAR approach and obtained a PCE of $4.22 \%$. It was shown that the re-organization of energy levels between CdS and CdSe forms a stepwise structure of band-edge levels, which is advantageous to the electron injection and hole-recovery of CdS and CdSe QDs. Since then, the CdS/CdSe core/shell QDs were widely used in QDSCs and a highest efficiency of 6.6\% was achieved based on this kind of QD sensitizer. ${ }^{192}$ Kim et al. prepared a $\mathrm{PbS} / \mathrm{CdS}$ core/shell QDs sensitized photoanode through SILAR method, showing a PCE of $3.25 \%$ with use of insulating oxide materials $\left(\mathrm{MgO}\right.$ and $\left.\mathrm{Al}_{2} \mathrm{O}_{3}\right)$ as barrier layer on bare $\mathrm{TiO}_{2}{ }^{272}$ 


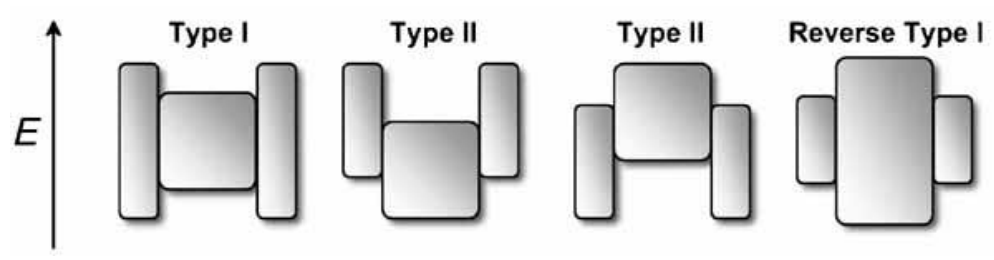

Fig. 13 Schematic representation of the energy-level alignment in different core/shell systems realized with semiconductor NCs to date. The upper and lower edges of the rectangles correspond to the positions of the conduction- and valence-band edge of the core (center) and shell materials, respectively. Reprinted with permission from ref. 270. Copyright (2009) Wiley.

It is noted that the SILAR or CBD method is not feasible to construct other kinds of core/shell QDs, especially for the Te based core/shell QDs due to the difficulty to prepare a stable Te precursor. In addition, the direct growth route is unable to obtain exact core/shell structured QDs due to the uncontrollability of nucleation and growth of the QDs in the confined mesoporous space. Therefore, a variety of core/shell QD sensitizers that used in QDSCs were pre-synthesized and then deposited on $\mathrm{TiO}_{2}$ electrode. On the basis of the band alignment of core and shell QDs, core/shell QDs can be classified into three types: type-I, reverse type-I, and type-II structure (Fig. 13). ${ }^{270}$ All of these three kinds of core/shell QDs have been investigated as sensitizers in QDSCs. For example, in a type-I structured QD, the $\mathrm{CB}$ of the shell material is higher than the core material and the shell is usually used to passivate the surface of the core so as to improve the optical properties and chemical stability. Parkinson et al. used type-I structured CdSe/ZnS QDs to sensitize single crystal $\mathrm{TiO}_{2}$ for the first time and found that the stability of the photoanode can be significantly improved compared to the core QDs based one. ${ }^{234}$ Zhong et al. prepared type-I structured "green" $\mathrm{CuInS}_{2} / \mathrm{ZnS}$ QDs through cation exchange approach. ${ }^{116}$ The thin $\mathrm{ZnS}$ layer was found to be beneficial for reducing the defect density of QDs and inhibiting charge recombination processes in the QDSCs (Fig 14a). As a result, an impressive record PCE of 7.04\% was obtained based on the $\mathrm{CuInS}_{2} / \mathrm{ZnS}$ QDs, whereas the plain $\mathrm{CuInS}_{2}$ QDs based cell only show a highest PCE of 5.05\%. Zhong's group also reported a type-I structured CdSeTe/CdS based QDSCs, exhibiting a PCE of 9.48\%, which is distinctly higher than the plain CdSeTe QDs based one $(8.02 \%)$. It was shown that the improved photovoltaic performance was mainly attributed to the suppressed charge recombination rates inside QDs and at the 
$\mathrm{QD} / \mathrm{TiO}_{2} /$ electrolyte interface due to the reduced trapping state defects in QDs. ${ }^{273}$
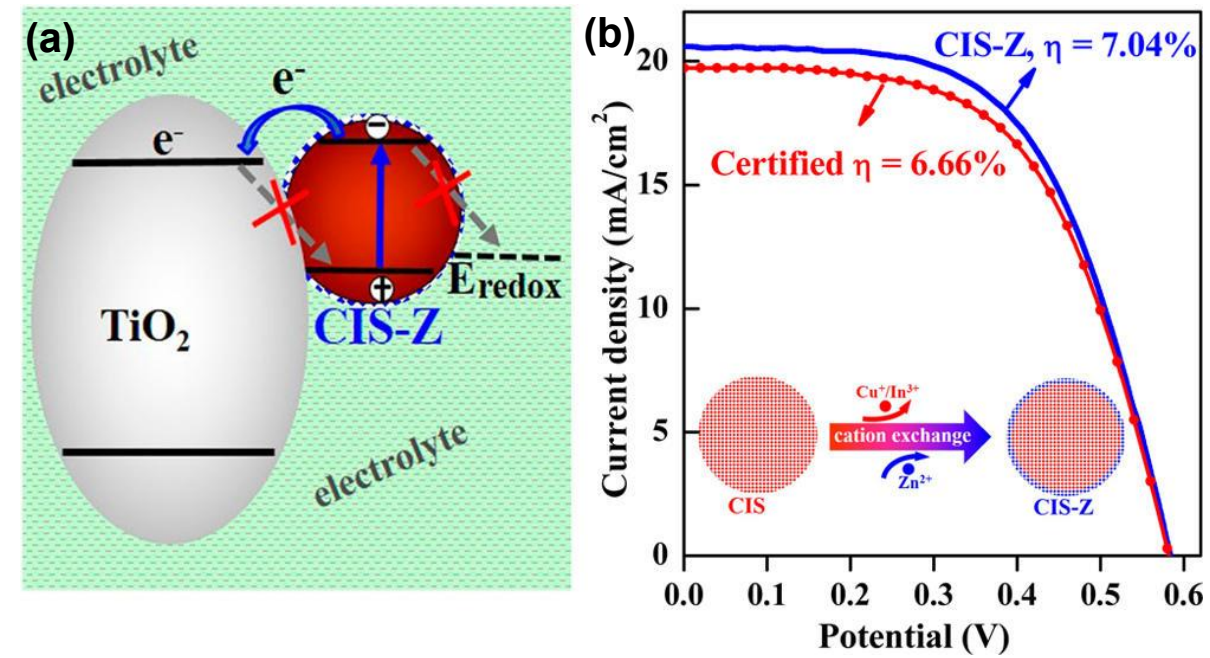

Fig. 14 (a) Schematic illustration of the charge recombination inhibition mechanism of $\mathrm{CuInS}_{2} / \mathrm{ZnS}$ QDSCs. (b) $J-V$ curves of $\mathrm{CuInS}_{2} / \mathrm{ZnS}$ based champion cells (blue) and the certified cell (red). Reprinted with permission from ref. 116. Copyright (2014) American Chemical Society.

In type-II structured core/shell QDs, both the CB and VB of the shell material are higher or lower than the core material. As a result, the electron and hole are spatially separated, enabling fast electron transfer from the QD sensitizer to the oxide matrix. ${ }^{270,271}$ What's more, type-II QDs show remarkable red shift of absorption edge due to the effect of exciplex state. These attractive properties enable type-II core/shell QDs a promising light harvesting material in QDSCs. For example, Ning et al. constructed type-II ZnSe/CdS core/shell QD sensitized solar cells, showing an efficiency of $0.27 \% .{ }^{217}$ Kuang's group reported a one-step linker-assisted $\mathrm{CBD}$ method to prepare $\mathrm{CdTe} / \mathrm{CdS}$ QD sensitized $\mathrm{TiO}_{2}$ electrodes, achieving an efficiency of $3.8 \%{ }^{215}$ Meng et al. applied a microwave assisted aqueous method to prepare type-II structured $\mathrm{CdSe}_{\mathrm{x}} \mathrm{Te}_{1-\mathrm{x}} / \mathrm{CdS}$ core/shell QDs, exhibiting a PCE of $5.04 \%{ }^{247}$ Zhong's group explored CdTe/CdSe type-II core/shell QDs as sensitizer in QDSCs with use of postsynthesis assembly approach and obtained a record PCE of 6.76\% (Fig 15a-d). ${ }^{74}$ It was demonstrated that the type-II core/shell QDs can not only extent the light absorption range of the solar cells, but also accelerate electron injection and suppress charge recombination processes. Furthermore, Zhong and coworkers developed another type-II core/shell QDs, $\mathrm{ZnTe} / \mathrm{CdSe}$, which possess much larger conduction band offset in comparison with that of $\mathrm{CdTe} / \mathrm{CdSe}$, as sensitizer and further improved the record PCE to $7.17 \%$ (Fig 15e). ${ }^{85}$ 

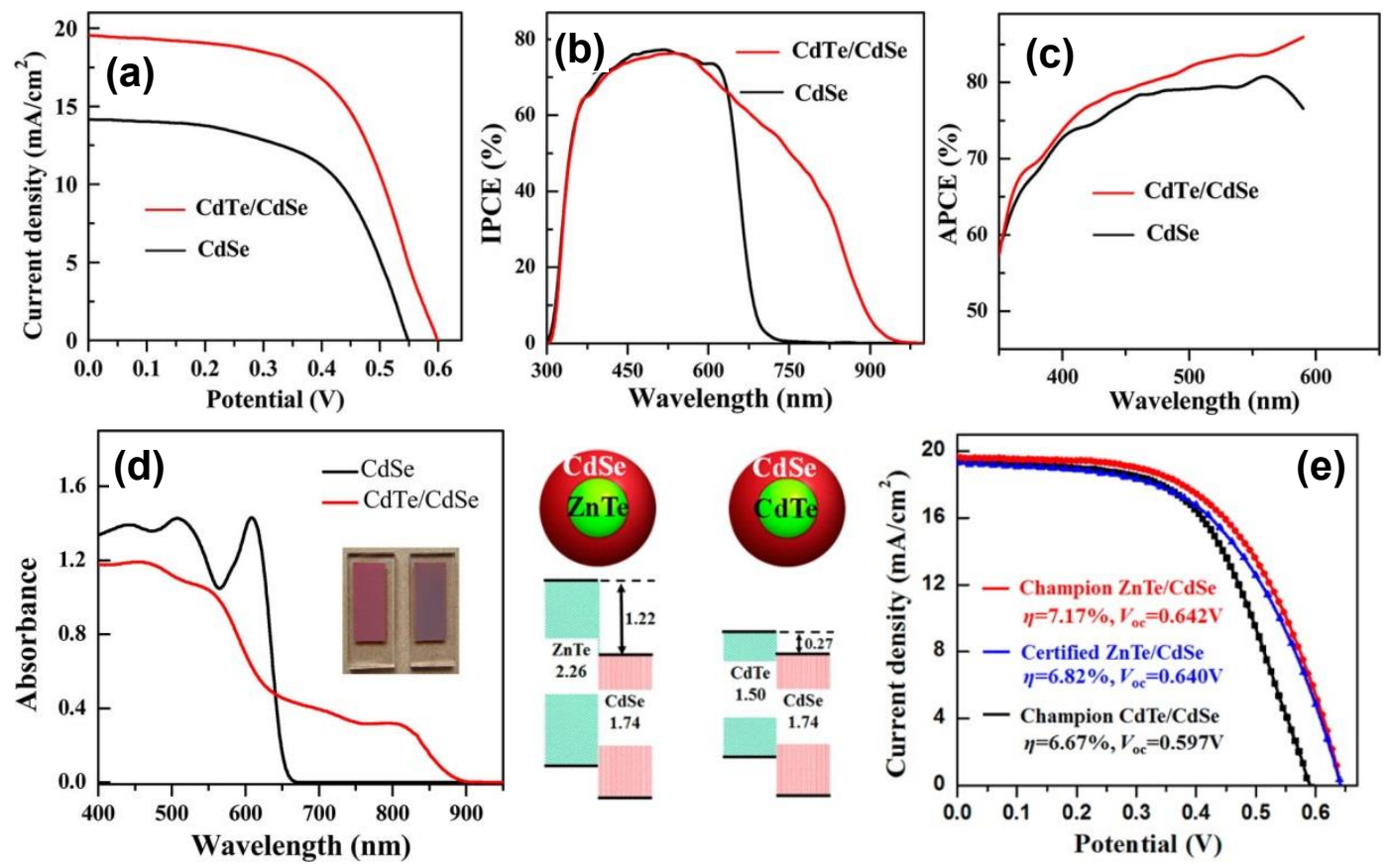

Fig. 15 (a) $J-V$, (b) IPCE, and (c) APCE curves of CdSe and CdTe/CdSe QD-based QDSCs. (d) Diffuse reflectance absorption spectra of identically sized $\mathrm{CdTe} / \mathrm{CdSe}$ and $\mathrm{CdSe}$ QD-sensitized $\mathrm{TiO}_{2}$ film electrodes. Insets: photographs of CdTe/CdSe (right) and CdSe (left) QD-sensitized $\mathrm{TiO}_{2}$ film electrodes. Reprinted with permission from ref. 74. Copyright (2013) American Chemical Society. (e) Schematic diagram of the band gap and band offsets (in eV) for the interfaces between bulk $\mathrm{ZnTe} / \mathrm{CdSe}$ and $\mathrm{CdTe} / \mathrm{CdSe}$ and the $J-V$ curves of the corresponding QDSCs. Reprinted with permission from ref. 85. Copyright (2015) American Chemical Society.

4.2.4. Alloyed QDs. Compared to binary QDs, ternary or quaternary alloyed QD are an attractive alternative to binary QD sensitizers since their optoelectronic properties can be tuned by controlling their composition without changing the particle size, and their band gap has the possibility to be narrower than their binary constituents due to the non-linear relationship between the composition and band gap. ${ }^{274-277}$ What's more, alloyed QDs also show higher chemical stability than their constituents due to the hardened lattice structure and decreased interdiffusion. ${ }^{274}$ 

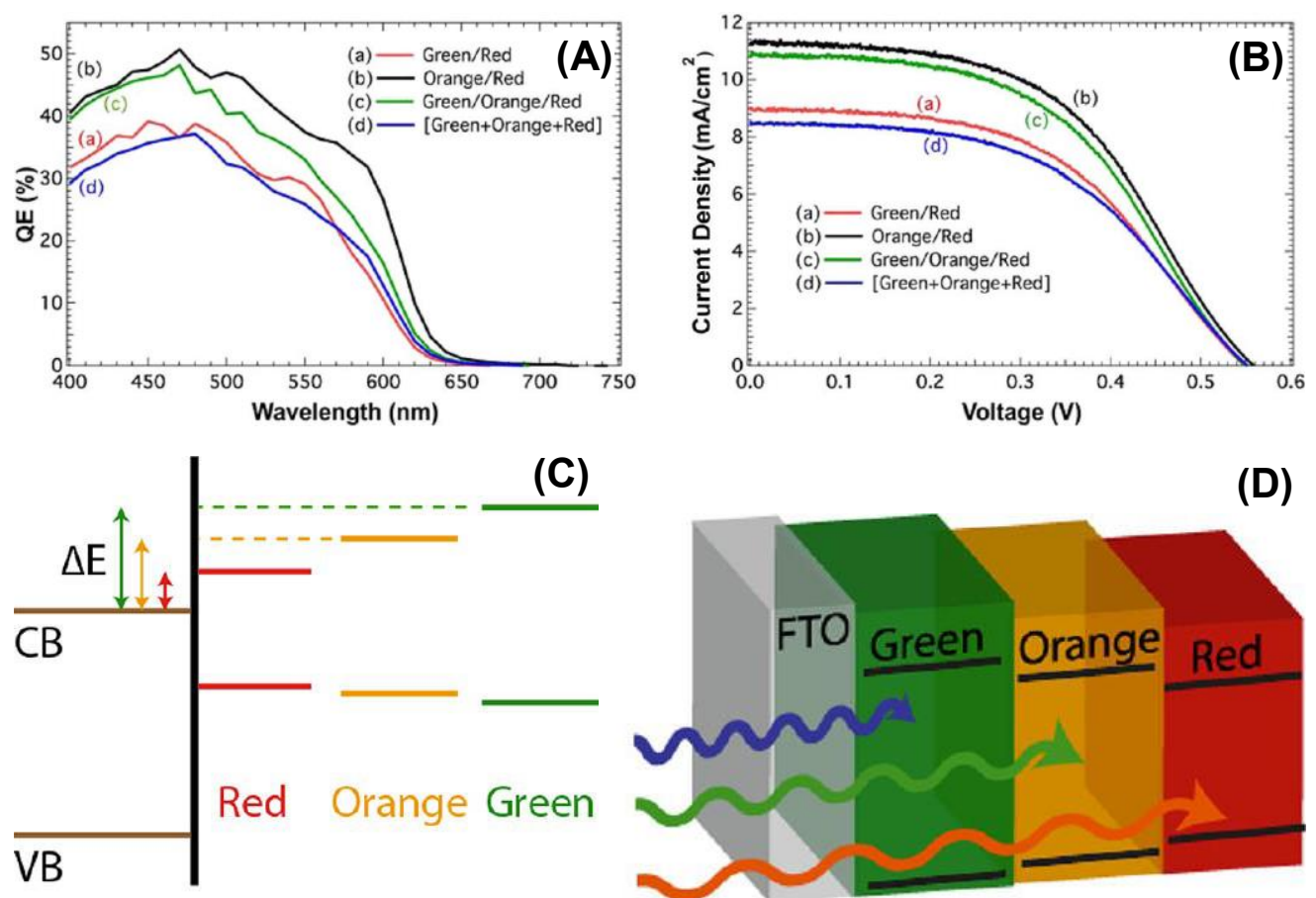

(D)

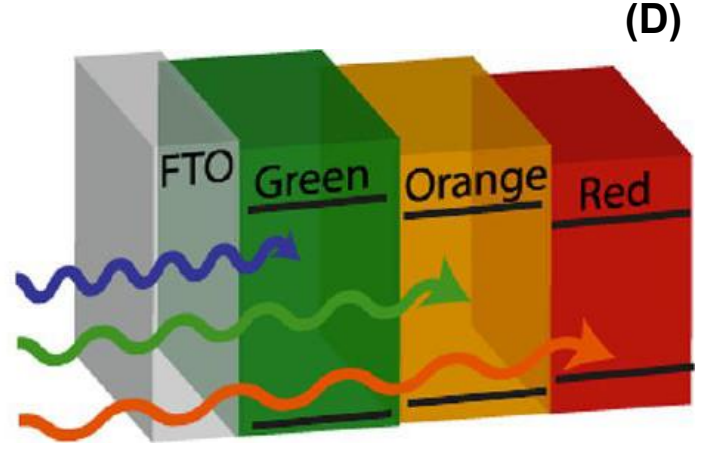

$\mathrm{TiO}_{2}$

CdSeS

Fig. 16 (A) IPCE spectra and (B) $J-V$ characteristics of different composite working electrodes containing (a) green/red, (b) orange/red, or (c) green/orange/red sequential layers or (d) a layer of premixed [green + orange + red] CdSeS QDs deposited by EPD. (C) Schematic illustration of the conduction and valence band positions of the green, orange, and red CdSeS QDs relative to $\mathrm{TiO}_{2}$. (D) Schematic illustration of the light absorption way in the tandem anode. Reprinted with permission from ref. 218. Copyright (2013) American Chemical Society.

The alloyed QDs used in QDSCs were mainly focused on II - VI $\left(\mathrm{CdS}_{\mathrm{x}} \mathrm{Se}_{1-\mathrm{x}}, \mathrm{CdSe}_{\mathrm{x}} \mathrm{Te}_{1-\mathrm{x}}\right.$ etc.) and I- III-VI ( $\mathrm{AgInS}_{2}, \mathrm{CuInS}_{2}, \mathrm{CuInSe}_{2}$ etc.) group QDs. Among these, $\mathrm{CdS}_{\mathrm{x}} \mathrm{Se}_{1-\mathrm{x}}$ alloyed QDs were first explored as sensitizer in QDSCs. For example, Wang et al. prepared CdSeS QDs in solution and deposited them on $\mathrm{TiO}_{2}$ nanowires for the first time, achieving a PCE of $0.6 \%{ }^{278}$ Hossain et al. deposited $\mathrm{CdS}_{\mathrm{x}} \mathrm{Se}_{1-\mathrm{x}}$ alloyed $\mathrm{QDs}$ on $\mathrm{TiO}_{2}$ by alternately depositing CdS and CdSe layers through SILAR approach, yielding a PCE of 4.05\%. ${ }^{279}$ Kamat et al. designed multiple absorber layers of CdSeS QDs with varying band gap within the $\mathrm{TiO}_{2}$ film. ${ }^{218}$ This multiple absorber architecture was found to be advantageous for improving light harvesting capability of the solar cells and PCEs of 3.2 and 3.0\% obtained for QDSCs with two and three layers of QDs, respectively (Fig. 16). The limitation of CdSeS 
QDs is their narrow light absorption range, resulting in relatively poor photovoltaic performance. As another II - VI group alloyed QDs, CdSe $\mathrm{Te}_{1-\mathrm{x}}$ QDs sensitizer, developed by Zhong's group show superior characteristics such as wide absorption range extending to near-IR region, high conduction band edge, and good chemical stability (Fig. 17). ${ }^{111}$ A record PCE of $6.36 \%$ was obtained in 2013 basing on this kind of alloyed QDs sensitizer. Since then, a series of record PCEs were reported basing on this kind of QDs. ${ }^{113,114,117,118}$
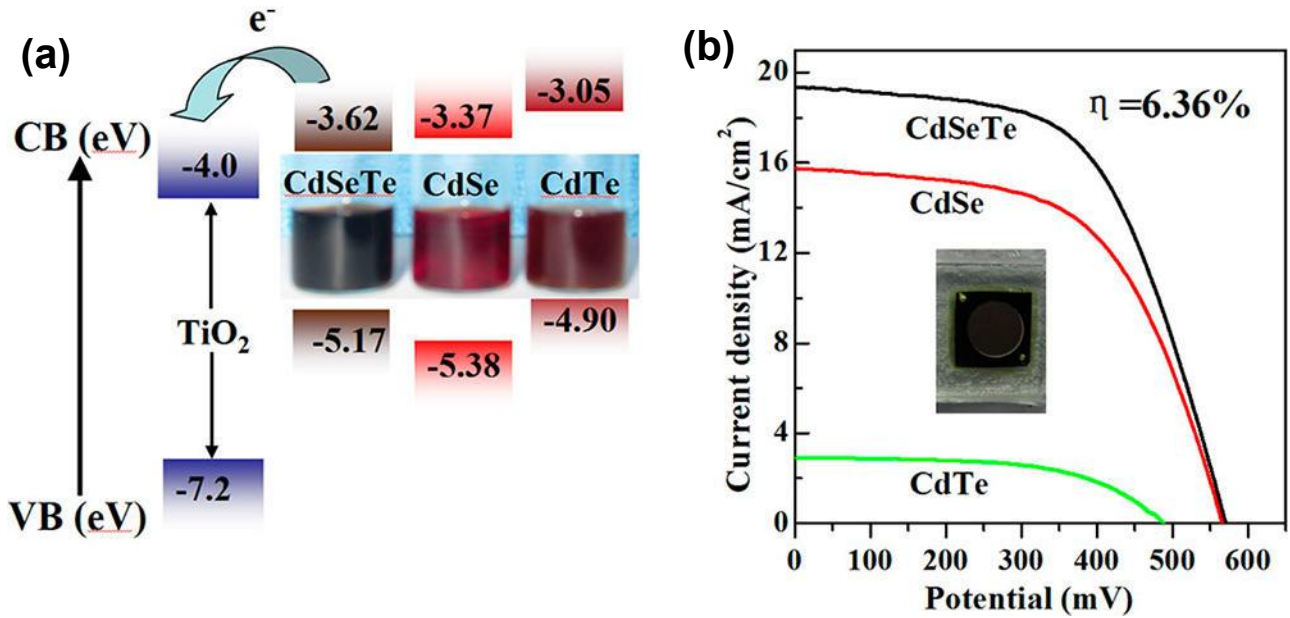

Fig. 17 (a) Schematic energy level diagram of $\mathrm{TiO}_{2}, \mathrm{CdSeTe}_{800}, \mathrm{CdSe}_{614}$, and $\mathrm{CdTe}_{680}$ QDs. (b) $J-V$ curves of QDSCs based on different QD sensitizers. Reprinted with permission from ref. 111. Copyright (2013) American Chemical Society.

I - III - VI group semiconductor, such as $\mathrm{CuInS}_{2}$ (CIS) and $\mathrm{CuInSe} \mathrm{I}_{2}$ (CISe), are considered as "green" light adsorption materials without $\mathrm{Cd}$ or $\mathrm{Pb}$ heavy metal elements. In addition, $\mathrm{I}-$ III - VI group semiconductor possesses prominent advantages such as high absorption coefficient and narrow band gap, rendering them exhibiting excellent photovoltaic performance in thin film solar cells. ${ }^{11,280-282}$ However, the fabrication of I - III - VI group light harvesting materials in thin film solar cells typically applied magnetron sputtering or thermal evaporation in vacuum condition, which are costly and high energy consumption. Therefore, in recent years, I - III - VI group QDs have attracted much attention in QDSC, which is a low-cost photovoltaic technology. ${ }^{77-79,221,242,246,283-293}$

In 2009, Chen et al. used CIS and CIS/ZnS QDs to sensitize ZnO nanowires, obtaining a 
PCE of $0.71 \%$ with use of $\mathrm{I}^{-} / \mathrm{I}_{3}{ }^{-}$electrolyte. ${ }^{288}$ Meng et al. synthesized CuInS 2 QDs in aqueous media and employed them as sensitizer in QDSCs, achieving a PCE of $1.47 \% .^{242}$ Teng's group proposed a heterojunction architecture with use of CIS and CdS QDs as sensitizers, boosting the PCE of CIS based QDSCs to $4.2 \%{ }^{287}$ It was shown that the introduction of CdS layer around CIS QDs can extend the optical absorption range and suppress charge recombination as well. Afterwards, Meng et al. doped Mn in the CdS layer, improving the PCE to $5.38 \% .{ }^{246}$ A key limitation of the CIS based QDSCs is the low QD loading amount, arising from the adopted dodecanethiol (DDT) ligand in the QD preparation. Since DDT cannot be completely displaced during the phase transfer procedure, this results in the difficulty in immobilizing QD on $\mathrm{TiO}_{2}$ film electrode. Zhong et al. developed a DDT-free synthetic approach for CIS QDs and constructed a type-I structured CIS/ZnS QDs through cation exchange approach, boosting the PCE of Cd-free CIS based QDSCs to 7.04\% (Fig. 14). ${ }^{116}$ Furthermore, they developed a $\mathrm{Zn}-\mathrm{Cu}-\mathrm{In}-\mathrm{S}$ alloyed QD sensitizer, exhibiting a PCE of $8.55 \% .{ }^{220}$ It was demonstrated that alloyed structure is superior to that of the CIS/ZnS core/shell and pristine CIS QDs as sensitizer benefiting from the suppression of charge recombination as well as acceleration of electron injection efficiency. Up to now, this is the best efficiency for CIS QDs based QDSCs.
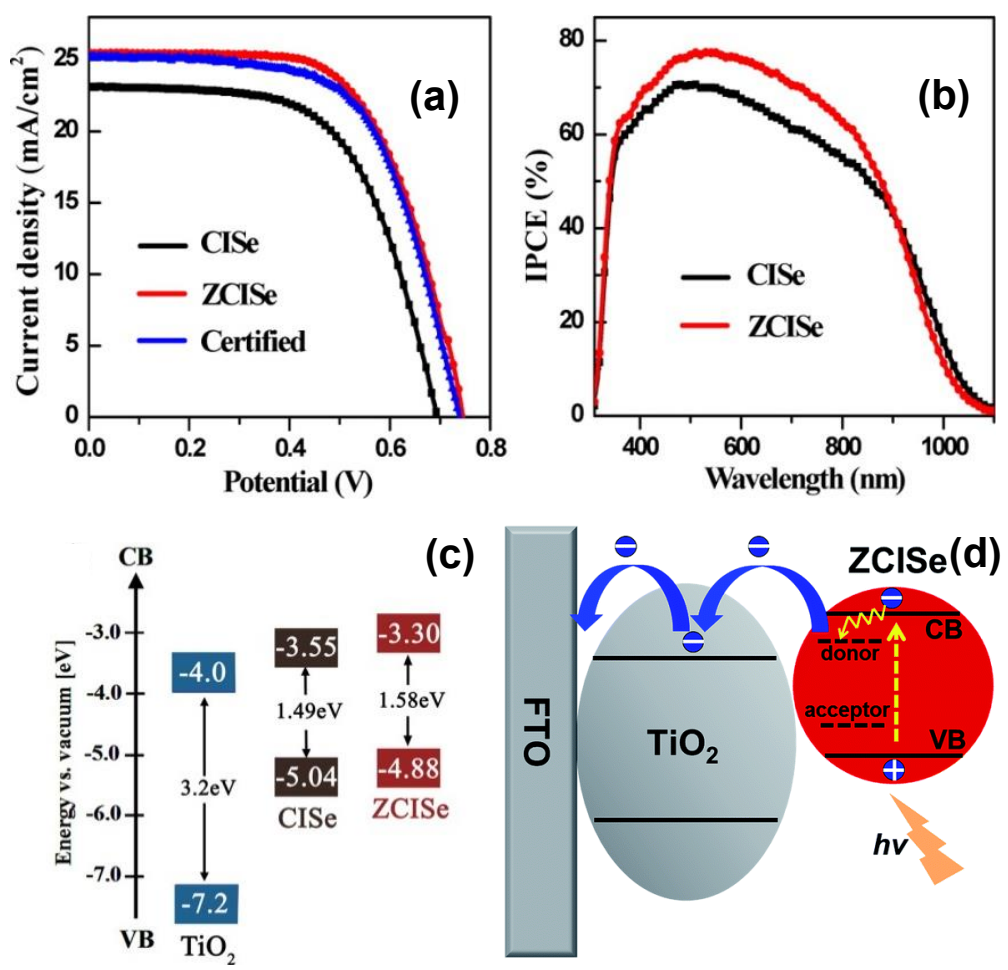

Fig. $18 J-V$ (a) and IPCE (b) curves for the champion cells of ZCISe and CISe QDs based QDSCs. (c) Schematic energy level diagrams of $\mathrm{TiO}_{2}, 4.1 \mathrm{~nm}$ CISe, and ZCISe QDs. 
Reprinted with permission from ref. 115. Copyright (2016) American Chemical Society. (d) Schematic diagram illustrating the effect of the donor-acceptor pair (DAP) in the QDs on the charge transfer process in the QDSCs. Reprinted with permission from ref. 222. Copyright (2017) Royal Society of Chemistry.

CISe QDs, which possess narrower band gap compared to CIS QDs (1.05 vs $1.52 \mathrm{eV})$, show great potential as sensitizer in QDSCs with possibility to extent light absorption edge to near-infrared region (NIR) and therefore obtaining higher $J_{\mathrm{sc}}$ value. ${ }^{280,294,295}$ Hyeon et al. presented a new synthetic process of CISe QDs through lewis acid-base reaction of metal iodides and selenocarbamate, showing a PCE of $4.3 \%$ for the Cd-free QDSCs. ${ }^{292}$ Klimov et al. reported a CuInSe ${ }_{1-\mathrm{x}} \mathrm{S}_{\mathrm{x}}$ alloyed I - III - VI group QD sensitizer and achieved a PCE of 5.5\% with the aid of a cation exchange process with $\mathrm{Zn}^{2+}$ or $\mathrm{Cd}^{2+}$ in the prepared CISe QDs. ${ }^{78,79}$ Hyeon et al. boosted the PCE of CISe QDSCs to 8.1\% through the optimization of $\mathrm{ZnS}$ passivation layer thickness and obtained a $J_{\mathrm{sc}}$ as high as $26.93 \mathrm{~mA} \mathrm{~cm}^{-2}{ }^{221}$ Notably, Zhong's group explored an alloyed $\mathrm{Zn}-\mathrm{Cu}-\mathrm{In}-\mathrm{Se}$ (ZCISe) QD sensitizer possessing narrow band gap and high conduction band edge simultaneously, achieving a record PCE of $11.61 \%$ combining with an Ti mesh supported mesoporous carbon counter electrode (Fig. 18a-c). ${ }^{115}$ It should be noted that this is the first report of QDSCs with PCE over 10\%. Afterwards, Zhong et al. found that the non-stoichiometry of the ZCISe QDs showed remarkable effects on the performance of the constructed QDSCs and the Cu-deficient ZCISe QDs were favorable for the improvement of the photovoltaic performance. ${ }^{222}$ The PCE of ZCISe QDs based QDSCs was further improved to $12.57 \%$ with the optimization of $\mathrm{Cu}$ content in QDs. They proposed that the enhanced PCE was mainly derived from the defect state-related donor-acceptor pair (DAP) in the ZCISe QDs due to the Cu deficiency (Fig. 18d).

According to the above mentioned QDs sensitizers, it is reasonable to conclude that the alloyed QDs, especially I- III - VI group alloyed QDs, seems to be a better choice as sensitizer in QDSCs. The reported PCEs over 10\% were mainly derived from I- III - VI group alloyed QDs sensitizers, taking benefit of the narrow band gap, the appropriate band edge, and the facile synthetic process of this kind of QDs. In addition, they also present a better environmental behavior than other QDs containing heavy metals. Therefore, it is believed that the I- III-VI group alloyed QDs possess great potential to promote the 
development of QDSCs furthermore in the near future.

4.2.5. Doped QDs. The introduction of dopant in QDs is another way to tune the electronic and photophysical properties of QDs. The effect of dopant on photoelectronic properties of QDs and the underlying mechanism has been widely studied. ${ }^{296-301}$ The effect of dopant in QDs sensitizer has also been investigated in QDSCs. ${ }^{107,185,191,203,246,302-312}$ In 2012, Kamat and coworkers introduced the doping strategy in QDs and studied doping effect on the photovoltaic performance in QDSCs (Fig. 19). ${ }^{107}$ They applied Mn dopant in CdS core layer through SILAR approach to form Mn:CdS/CdSe QDs sensitizers. The Mn d-d transition is both spin and orbital forbidden, resulting a long electron lifetime. This unique feature is believed to favor prolonging lifetime of the photo-generated electrons. As a result, an impressive PCE of 5.4\% was obtained and the cell showed good stability as well. Meng et al. prepared CIS QDs first in aqueous media and then fabricated CIS/Mn:CdS sensitized photoanode by SILAR approach. ${ }^{246}$ The obtained PCE for the QDSCs based on the doped QDs (5.38\%) was higher than the undoped one (4.69\%). Santra et al. investigated the role of Mn dopant in QDSCs. ${ }^{307}$ It was demonstrated that the Mn dopant decreases the recombination within the QDs. The long lifetime of the photoexcited electron eventually improves the $J_{\mathrm{sc}}$ and $V_{\mathrm{oc}}$ values by raising the Fermi energy to higher negative potential. Zhong et al. applied Mn dopant in both the pre-synthesized $\mathrm{CdSe}_{\mathrm{x}} \mathrm{Te}_{1-\mathrm{x}}$ QDs sensitizer and the $\mathrm{ZnS}$ passivation layer, improving the PCE of the corresponding QDSCs from $8.55 \%$ to $9.40 \%{ }^{312}$ Experimental results demonstrated that the presence of Mn dopant can reduce trap states in QD sensitizers, consequently suppressing the charge recombination process in QDSCs. Apart from Mn dopant, other dopant such as Co has also been used in QDs sensitizer. For example, Firoozi et al. investigated the effect of $\mathrm{Co}^{2+}$ ion incorporation into $\mathrm{CdS} / \mathrm{CdSe}$ QDs on the performance of QDSCs. The PCE can be distinctly improved from $2.33 \%$ to $3.16 \%$ with the optimization of Co doping amount. ${ }^{309}$
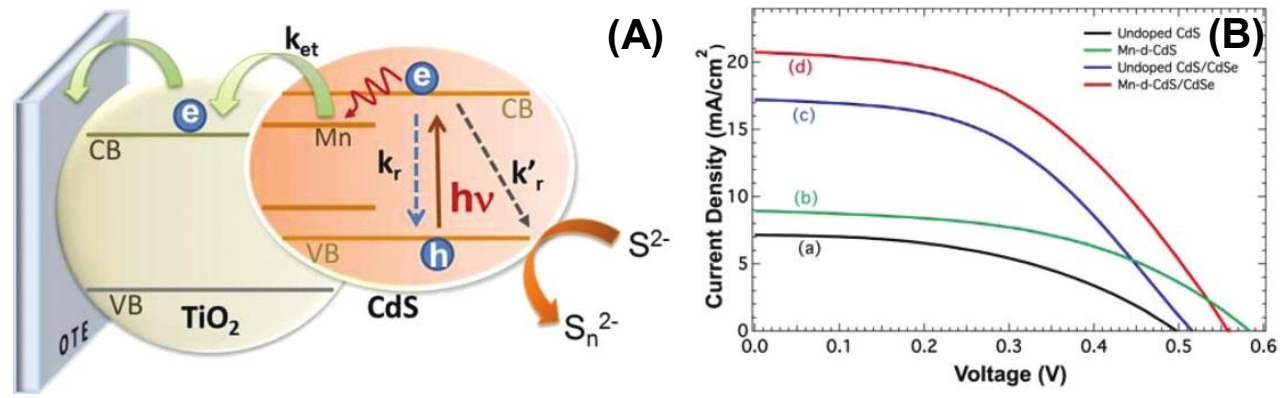

Fig. 19 (A) Schematic diagram illustrating the electron transfer $\left(k_{\mathrm{et}}\right)$ from doped CdS into 
$\mathrm{TiO}_{2}$ nanoparticles. $k_{\mathrm{r}}$ and $k_{\mathrm{r}}^{\prime}$ represent electron recombination with holes and redox couple, respectively. (B) $J-V$ characteristics of different working electrodes measured under AM 1.5 global filter of $100 \mathrm{~mW} \mathrm{~cm}{ }^{-2}$ sunlight: (a) undoped CdS, (b) Mn-doped-CdS, (c) undoped $\mathrm{CdS} / \mathrm{CdSe}$, and (d) Mn-doped-CdS/CdSe. Reprinted with permission from ref. 107. Copyright (2012) American Chemical Society.

4.2.6. Other QDs sensitizers. Apart from the abovementioned QDs sensitizers, there are several novel sensitizers have also been explored in QDSCs. For example, Kamat et al. found that gold clusters with diameters of 1.0-1.6 nm can also inject electrons into $\mathrm{TiO}_{2}$ under visible excitation. ${ }^{211}$ The fabricated QDSCs based on the gold clusters exhibited a PCE of $2.36 \%$ with use of $\mathrm{Co}(\mathrm{II}) / \mathrm{Co}(\mathrm{III})$ redox couple as electrolyte. Bang et al. investigated the effect of the size of Au clusters on the performance of the sensitized solar cells. ${ }^{313}$ They constructed the cell devices using $\mathrm{I}^{-} / \mathrm{I}_{3}{ }^{-}$as electrolyte and obtained a PCE of $3.8 \%$ with use of $\mathrm{Au}_{18}(\mathrm{SR})_{14}$ as sensitizer due to its relatively good light absorption capability and low recombination rate.

Another kind of novel sensitizer is carbon QD (CQD), which is more low-cost and environmental friendly. ${ }^{212,314-319}$ In 2010, Li et al. synthesized solution-processable, black graphene QDs and applied them as sensitizer for solar cell. ${ }^{318}$ The calculated band edge position of the graphene QDs was found to be suitable for electron injection and QDs regeneration. The fabricated solar cell exhibited a $J_{\mathrm{sc}}$ of $0.2 \mathrm{~mA} \mathrm{~cm}^{-2}$, a $V_{\mathrm{oc}}$ of $0.48 \mathrm{~V}$, and a FF of 0.58. Ozin et al. synthesized CQDs via the dehydration of $\gamma$-butyrolactone with concentrated sulphuric acid. ${ }^{317}$ The CQDs were employed as sensitizer in QDSCs and achieved a PCE of $0.13 \%$. Zhang et al. present a simple in situ strategy of growing CQDs directly onto $\mathrm{TiO}_{2}$ surfaces, obtaining an impressive PCE of $0.87 \%$, which is the best PCE for CQDs based QDSCs. ${ }^{319}$
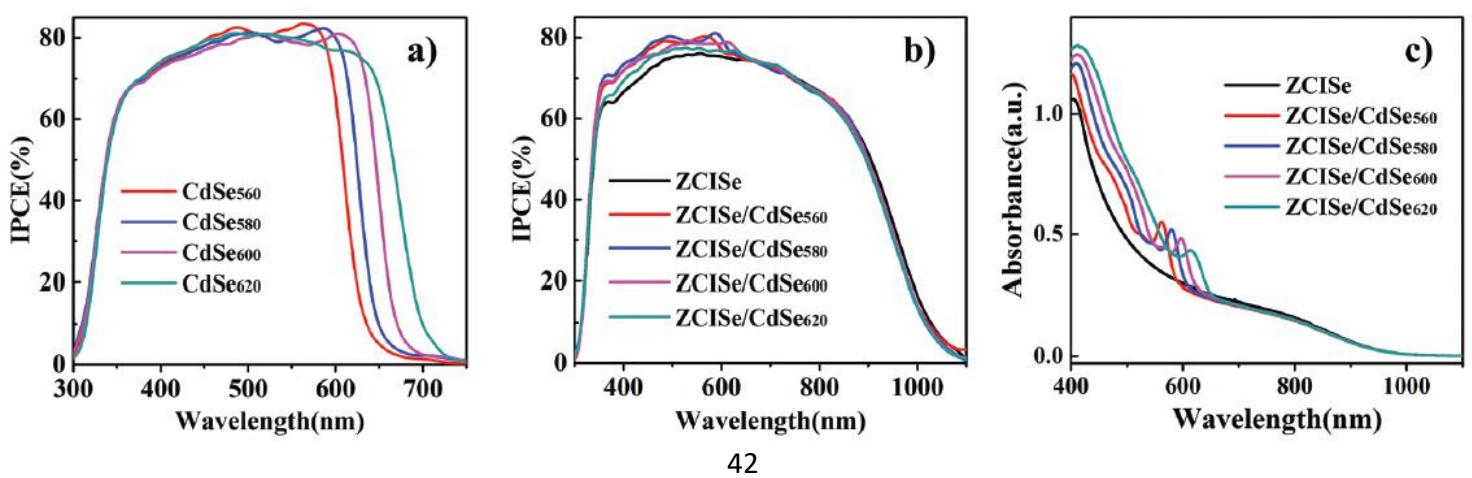
Fig. 20 IPCE spectra of QDSCs cosensitized with various photoanodes: (a) CdSe QDs based QDSCs; (b) ZCISe- and ZCISe/CdSe-based QDSCs. (c) UV-vis absorption spectra of ZCISe and ZCISe/CdSe QD-sensitized $\mathrm{TiO}_{2}$ film electrodes with an active area of $1.6 \mathrm{~cm}^{2}$. Reprinted with permission from ref. 112. Copyright (2018) Wiley.

4.2.7. Co-sensitization strategy. Unlike the case of DSC, the coverage of QDs on $\mathrm{TiO}_{2}$ is usually not complete and the reported highest coverage of QDs is only about $34 \% .{ }^{110}$ That is to say, a large portion of the $\mathrm{TiO}_{2}$ surface is bare and expose to electrolyte directly. ${ }^{36}$ The low coverage of QDs will lead to the decrease of light harvesting efficiency and the aggravation of charge recombination. One strategy to improve the QDs loading amount is co-sensitization with use of different QDs or dye molecule. What's more, co-sensitization strategy with use of different light harvesting material can achieve a higher light harvesting efficiency since different materials have different properties such as light absorption range and extinction coefficient. $^{32,240,320-327}$

For example, in 2008, Han et al. applied two different sized CdSe QDs to co-sensitize vertically aligned $\mathrm{TiO}_{2}$ nanotubes, obtaining a PCE of $1.2 \%$, which is higher than the single sized CdSe QDs sensitized cells. ${ }^{240}$ The improved PCE was mainly derived from the increase of $J_{\mathrm{sc}}$ values due to the broader light absorption range. Lei et al. achieved similar result with depositing two different sized $\mathrm{CdSe}$ QDs on $\mathrm{TiO}_{2}$ electrode. ${ }^{323}$ It was shown that improved photovoltaic performance can be attributed to the broader light absorption range and the reduced charge recombination loss at the interface due to the better coverage of CdSe QDs on the $\mathrm{TiO}_{2}$ film. Qiao et al. also demonstrated the effectiveness of this co-sensitization strategy on improving solar cell performance with use of dual-sized CdS QDs as sensitizers. ${ }^{321}$ Recently, Zhong et al. displayed a co-sensitization strategy employing $\mathrm{Zn}-\mathrm{Cu}-\mathrm{In}-\mathrm{Se}$ (ZCISe) and CdSe QDs as sensitizers (Fig. 20). ${ }^{112}$ The synergistic effect of ZCISe QDs with a wide light absorption range and CdSe QDs with a high extinction coefficient contributed to the improvement of light harvesting efficiency as well as the inhibition of charge recombination. As a result, a new record PCE of $12.75 \%\left(V_{\mathrm{oc}}=0.752 \mathrm{~V}, J_{\mathrm{sc}}=27.39 \mathrm{~mA} \mathrm{~cm}{ }^{-2}, \mathrm{FF}=0.619\right)$ is achieved for QDSCs. Meanwhile, metal-semiconductor nanohybrid materials (NHMs) were also used in enhancing light harvesting capability and photovoltaic performance of the resulting cells by Ghosh and coworkers. ${ }^{328}$ Experimental results showed that the absorption onset of $\mathrm{CdSe} / \mathrm{Au}$ can be red-shifted to $800 \mathrm{~nm}$. Higher recombination resistance and longer 
electron lifetime were also confirmed by EIS measurements at $\mathrm{TiO}_{2}-\mathrm{CdSe} / \mathrm{Au}$ interface in comparison with $\mathrm{TiO}_{2}-\mathrm{CdSe}$. Consequently, PCE of $4.39 \%$ was obtained for $\mathrm{CdSe} / \mathrm{Au}$ QDSC, which is $\sim 30 \%$ higher related to plain CdSe QDSC. 

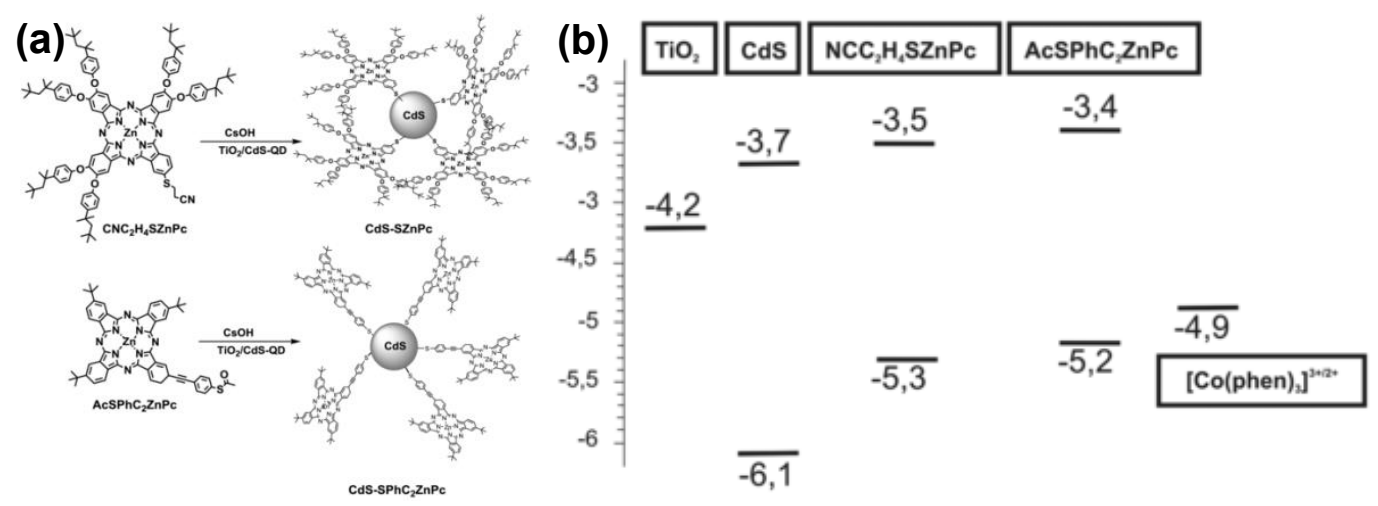

Fig. 21 (a) Preparation of CdS-SZnPc and $\mathrm{CdS}-\mathrm{SPhC}_{2} \mathrm{ZnPc}$ hybrid systems. (b) Energy levels of $\mathrm{TiO}_{2}, \mathrm{CdS}, \mathrm{CNC}_{2} \mathrm{H}_{4} \mathrm{SZnPc}, \mathrm{AcSPhC}_{2} \mathrm{ZnPc}$ and $\left[\mathrm{Co}(\text { phen })_{3}\right]^{3+/ 2+}$. Reprinted with permission from ref. 320. Copyright (2015) Wiley.

The co-sensitization strategy can also be carried out with use of QDs and organic dyes as sensitizers. ${ }^{320,324-327}$ Zaban and coworkers constructed a QDs and dye co-sensitization photoanode and shown that the QDs can serve as "antennas" to funnel absorbed energy to nearby dye molecules via FRET. ${ }^{326}$ The incorporation of QDs and dye can significantly enhance light absorption and broaden the absorption range. Kamat and coworkers employed CdS QDs and a NIR absorbing squaraine dye (JK-216) as co-sensitizers, delivering a PCE of $3.14 \%$, which is distinctly higher the individual sensitizer based ones. ${ }^{325}$ Sastre-Santos and coworkers fabricated a cascade co-sensitization photoanode with use of CdS QDs and zinc phthalocyanines (ZnPcs) dyes (Fig. 21). ${ }^{320}$ Unlike the previously co-sensitization method, in which QDs and dyes were separated by a barrier layer or the dyes were anchored to $\mathrm{TiO}_{2}$, the dyes in this work were directly anchored on CdS QDs through the thiol (SH) group in the dye. It was demonstrated that this cascade co-sensitization can not only expand the light absorption range, but also benefit the reduction of charge recombination since the dye acted as a passivating agent. Finally, the PCE was increased from $0.8 \%$ to $2.5 \%$ for the cells using single CdS QD and co-sensitizers, respectively. Overall, the obtained photovoltaic performance of the QDSCs relating to the co-sensitization strategy with use of QDs and organic dyes are relatively poor. This can be mainly ascribed to the limitation of the choice of QDs as well as the electrolyte that can stabilize both of the QDs and dyes. Further work about this strategy should focus on the exploration of superior QDs and suitable liquid or solid-state redox couple. 


\subsection{Counter electrode (CE)}

The main function of $\mathrm{CE}$ in QDSC is to catalyze the reduction reaction of oxidized electrolyte by electron from external circuit. ${ }^{7,42,51}$ A preferred CE should possess the following properties: (1) good conductivity to transfer the electrons from external circuit to the active sites; (2) high catalytic activity towards the reduction reaction of oxidized electrolyte; (3) good chemical and mechanical stability to ensure the whole stability of the device. The studied CE catalytic materials for QDSCs can be mainly divided into four kinds: noble metals, metal chalcogenides, carbon materials, and composite materials. The representative photovoltaic performances for QDSCs based on different CEs are summarized in Table 4. CE was one of the biggest limitations of the QDSC performance at the early stages and its continuous optimization has contribute significantly to the last increases reported in the cell efficiency.

4.3.1. Noble metals. In DSCs, thermal deposited Pt electrode is the most common CE due to its superior catalytic ability for the reduction of $\mathrm{I}^{-} / \mathrm{I}_{3}{ }^{-}$electrolyte. Accordingly, this classical CE is borrowed to QDSCs. ${ }^{48,49,51}$ For instance, Lee et al. employed Pt CE to construct CdS/CdSe QDSCs in 2009 and obtained a record PCE of 3.7\%. ${ }^{101}$ Kuang et al. achieved a PCE of $4.81 \%$ in 2011 by applying a Pt CE in electrodeposited CdS/CdSe QDSCs. ${ }^{233}$ Yang et al. showed a PCE of 5.25\% for Pt CE based QDSCs sensitized with $\mathrm{ZnSe} / \mathrm{CdSe} / \mathrm{ZnSe}$ quantum well. ${ }^{188}$ To the best of our knowledge, this is the best efficiency for noble metal CEs based QDSCs up to now. Besides Pt, researchers found that another noble metal $\mathrm{Au}$ is more superior as CE catalytic material. ${ }^{101,215,329-332}$ For example, Lee et al. showed that under the identical conditions, the PCE of QDSCs employing Au CE (4.22\%) was significantly higher than that of the Pt based one $(3.70 \%) .{ }^{101}$ The improvement of the photovoltaic performance was mainly attributed to the weak adsorption of $\mathrm{S}^{2-}$ to the $\mathrm{Au}$ surface as confirmed by cyclic voltammetry test (Fig. 22). Kuang et al. also demonstrated the superiority of $\mathrm{Au}$ relative to $\mathrm{Pt}$ as $\mathrm{CE}$ catalytic material in QDSCs. ${ }^{215}$ 


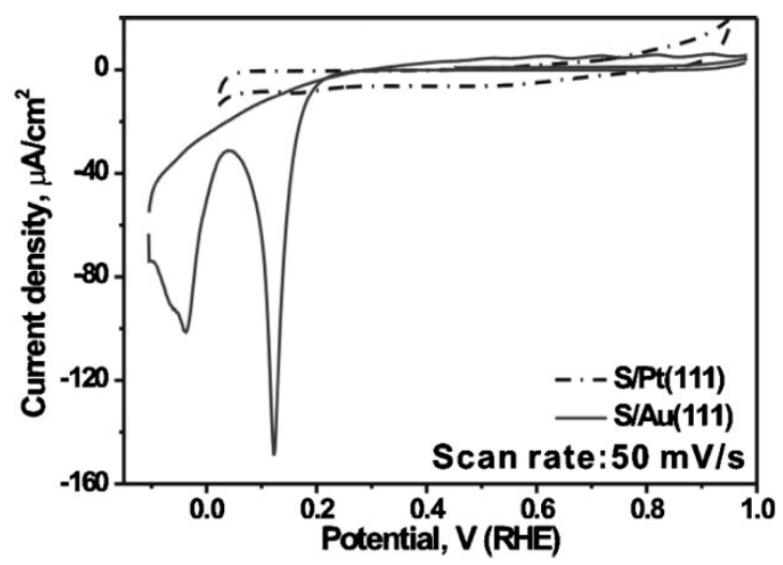

Fig. 22 Cyclic voltammograms for S-modified $\mathrm{Au}(111)$ and $\mathrm{Pt}(111)$ electrodes recorded at 50 $\mathrm{mV} \mathrm{s}^{-1}$ in $0.1 \mathrm{M} \mathrm{HClO}_{4}$ solution. Reprinted with permission from ref. 101. Copyright (2009) Wiley.

Typically, QDSCs based on noble metal CE exhibited moderate PCEs. This is mainly induced by the adsorption of $\mathrm{S}^{2-}$ onto the surface of $\mathrm{Pt}$ or $\mathrm{Au}$, leading to the poisoning of the $\mathrm{CE}$ and reducing its catalytic activity as well as the stability of the cell device. ${ }^{51}$ What's more, the high price of the noble metals increase the production cost of the device, thus limiting its commercial application. Therefore, much effort has been devoted to develop efficient and low-cost CEs in QDSCs.

4.3.2. Metal chalcogenides. As early as 1980, Hodes et al. has pointed out that metal sulfides were more suitable catalysts for the polysulfide redox reaction compared to the conventional Pt CE. ${ }^{333}$ Afterwards, it is indeed demonstrated that metal chalcogenide is a superior material for the fabrication of CE in QDSCs. Among these, copper chalcogenide is the most widely studied CE in QDSCs. Mora-Seró and Toyoda and coworkers carried out the attempt to fabricate QDSCs with use of $\mathrm{Cu}_{2} \mathrm{~S} C E$ and found that the PCE can be improved from $0.65 \%$ (Pt $\mathrm{CE}$ ) to $1.83 \%\left(\mathrm{Cu}_{2} \mathrm{~S} \mathrm{CE}\right) .{ }^{229}$ The improvement was derived from the reduction in the charge transfer resistance at the $\mathrm{CE} /$ electrolyte interfaces, affecting dramatically the fill factor of the device. Afterwards, Shen and Toyoda and coworkers reported a brass substrate based $\mathrm{Cu}_{2} \mathrm{~S} C E$ (simplified as $\mathrm{Cu}_{2} \mathrm{~S} /$ brass), which can be facilely prepared by directly immersing the brass foil in polysulfide electrolyte. ${ }^{122}$ They obtained a PCE of $1.8 \%$ based on CdSe QDs sensitized $\mathrm{TiO}_{2}$ nanotube working electrode. It should be noted that this brass based $\mathrm{Cu}_{2} \mathrm{~S} \mathrm{CE}$ was the most common $\mathrm{CE}$ in QDSCs since then and most of the high PCEs were derived from this CE based system. ${ }^{74,85,106,111,113,116,117,203}$ Up to now, 
the highest reported PCE for $\mathrm{Cu}_{2} \mathrm{~S} /$ brass based QDSCs is $10.75 \%$ basing on ZCISe QD sensitizers. ${ }^{112}$ Nevertheless, the problem of this $\mathrm{Cu}_{2} \mathrm{~S} / \mathrm{brass} \mathrm{CE}$ is that the polysulfide electrolyte can continually react with brass substrate, leading to the poor long term stability of CE as well as the resultant device.

Aiming at resolving the stability issue of $\mathrm{Cu}_{2} \mathrm{~S} /$ brass $\mathrm{CE}$, much work has focused on the preparation of $\mathrm{Cu}_{2} \mathrm{~S}$ on rigid substrate through electrodeposition, spray pyrolysis or screen printing etc. approaches to facilitate the sealing of the device. ${ }^{334-351}$ For example, Zhong and coworkers prepared a $\mathrm{Cu}_{2} \mathrm{~S} / \mathrm{FTO} \mathrm{CE}$ by electrodeposition of the copper film via a multipotential step technique, followed by dipping into polysulfide methanol solution to form $\mathrm{Cu}_{2} \mathrm{~S}^{349}$ This electrodeposited $\mathrm{Cu}_{2} \mathrm{~S} / \mathrm{FTO}$ CE based QDSCs exhibited a PCE of 5.21\%, which is comparable to that of the $\mathrm{Cu}_{2} \mathrm{~S} /$ brass $\mathrm{CE}$ based one (5.41\%). Especially, the stability of the cell under one sun illumination was significantly improved for over $10 \mathrm{~h}$ without performance degradation. Selopal et al. showed a spray pyrolysis approach to prepare $\mathrm{Cu}_{2} \mathrm{~S} / \mathrm{FTO} \mathrm{CE}$ and achieved a PCE of $3.75 \% .{ }^{345}$ The spray pyrolysis deposition method possesses the advantages of simple, cheap, time-saving, and good reproducibility. Wang et al. reported a novel method to prepare $\mathrm{Cu}_{2} \mathrm{~S} / \mathrm{ITO} \mathrm{CE}$ by ion exchange. ${ }^{340}$ They firstly deposited $\mathrm{ZnS}$ on ITO substrate through SILAR approach, followed by immersing into a saturated $\mathrm{CuCl}$ acetonitrile solution for ion exchange to form $\mathrm{Cu}_{2} \mathrm{~S}$ film. A final PCE of $4.78 \%$ was achieved, which is comparable to that of the $\mathrm{Cu}_{2} \mathrm{~S} /$ brass $\mathrm{CE}$ based one. Meng et al. prepared a $\mathrm{Cu}_{2} \mathrm{~S} / \mathrm{FTO} \mathrm{CE}$ through screen printing method with use of $\mathrm{Cu}_{2} \mathrm{~S}$ nanoparticle paste, exhibiting a PCE of $3.71 \% .{ }^{351}$ Zhang et al. used $\mathrm{CuS} / \mathrm{Cu}_{1.8} \mathrm{~S}$ composite nanomaterials to fabricate $\mathrm{Cu}_{\mathrm{x}} \mathrm{S} / \mathrm{FTO} \mathrm{CE}$, obtaining a PCE of $6.28 \%$, which is slightly higher than the $\mathrm{Cu}_{2} \mathrm{~S} / \mathrm{brass} \mathrm{CE}$ based one. ${ }^{350}$ What's more, the constructed cell showed good stability over $225 \mathrm{~h}$ benefitting from the good mechanical and chemical stability of the CE. 


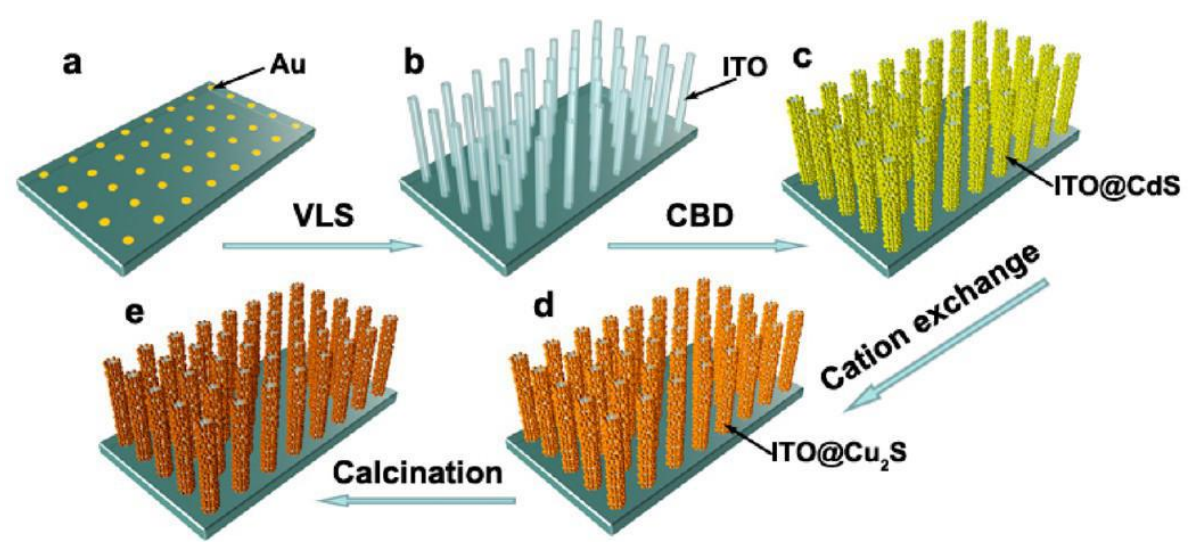

Fig. 23 Scheme for directly preparing ITO@ $\mathrm{Cu}_{2} \mathrm{~S}$ nanowire arrays on FTO glass. (a) Sputtering Au catalysts on FTO substrate; (b) CVD synthesis of ITO nanowire arrays; (c) chemical bath deposition of CdS shell on ITO nanowire arrays; (d) cation exchange to form ITO@ $\mathrm{Cu}_{2} \mathrm{~S}$ nanowire arrays; (e) calcination for improving the interfaces between ITO nanowire core and $\mathrm{Cu}_{2} \mathrm{~S}$ nanocrystal shell. Reprinted with permission from ref. 347. Copyright (2014) American Chemical Society.

Wan and coworkers developed a novel ITO/ $\mathrm{Cu}_{2} \mathrm{~S}$ nanowire arrays $\mathrm{CE}$ for QDSCs. ${ }^{33,346,347}$ As shown in Fig. 23, ITO nanowire arrays were first grown on Au-coated FTO substrate via CVD and then a layer of CdS in a thickness of about $20 \mathrm{~nm}$ was coated on the as-prepared ITO nanowires via CBD technique. After that, ITO/ $\mathrm{Cu}_{2} \mathrm{~S}$ nanowire arrays were formed through a solution-based cation exchange. The constructed QDSC showed a PCE of 4.06\%, which is significantly higher compared to the conventional $\mathrm{Cu}_{2} \mathrm{~S} / \mathrm{FTO} \mathrm{CE}$ based one (3.04\%). ${ }^{347}$ The improvement of the photovoltaic performance can be ascribed to the decrease in sheet resistance and charge transfer resistance benefitting from the high-quality tunnel junctions formed between n-type ITO nanowires and p-type $\mathrm{Cu}_{2} \mathrm{~S}$ nanocrystals, as well as the more active catalytic sites due to the three-dimensional structure of nanowire arrays. In addition, they further explored hierarchically assembled ITO/ $\mathrm{Cu}_{2} \mathrm{~S}$ nanowire arrays $\mathrm{CE}$ for the deposition of more $\mathrm{Cu}_{2} \mathrm{~S}$ nanocrystals as active sites, boosting the PCE to $6.12 \%$ with use of CdSeTe QDs as sensitizer. ${ }^{346}$

Apart from $\mathrm{Cu}_{2} \mathrm{~S}, \mathrm{CuSe}$ or $\mathrm{Cu}_{2} \mathrm{Se}$ were also applied to fabricate CE in QDSCs. ${ }^{352-357}$ For example, Cui et al. prepared $\mathrm{Cu}_{\mathrm{x}} \mathrm{Se} / \mathrm{FTO} \mathrm{CE}$ through a novel route. ${ }^{353}$ They firstly deposited red selenium on FTO by disproportionation of $\mathrm{Na}_{2} \mathrm{SeSO}_{3}$ aqueous solution with an ethanol spray and then the red selenium is manipulated as both template and reaction center to fabricate a cuprous embedded $\mathrm{Cu}_{\mathrm{x}} \mathrm{Se}$ composite $\mathrm{CE}$ upon selenium self-redox reaction with 
copper ions. The constructed QDSCs exhibited a PCE of 3.80\%, which is slightly higher than the $\mathrm{Cu}_{2} \mathrm{~S} /$ brass $\mathrm{CE}$ based one (3.72\%). Bang and coworkers prepared various metal selenide CEs by SILAR method and investigated their performance in QDSCs. ${ }^{352}$ It was found that $\mathrm{Cu}_{1.8} \mathrm{Se}$ based $\mathrm{CE}$ was particularly superior to the other kinds of metal selenides based CEs, achieving a PCE of $5.0 \%$. Tian and coworkers developed a $\mathrm{Cu}_{3} \mathrm{Se}_{2} \mathrm{CE}$ composed of nanorod arrays and nanosheets, delivering a PCE of $5.05 \%$, higher than the $\mathrm{Cu}_{2} \mathrm{~S} /$ brass based one $(4.10 \%) .{ }^{354}$ Wang and coworkers prepared $\mathrm{Cu}_{2-\mathrm{x}} \mathrm{Se}$ nanoparticles and nanowires catalysts by facile wet-chemical methods and then used as CEs in QDSCs. ${ }^{356}$ The results showed that $\mathrm{Cu}_{2-\mathrm{x}} \mathrm{Se}$ nanoparticle based $\mathrm{CE}$ can remarkably enhance the efficiency by $17.1 \%(6.50 \%$ vs $5.55 \%$ ) compared to $\mathrm{Cu}_{2} \mathrm{~S} \mathrm{CE}$. Zhong and coworkers deposited $\mathrm{Cu}_{\mathrm{x}} \mathrm{Se}$ nanoparticles on FTO substrate and found that the copper deficient $\mathrm{Cu}_{\mathrm{x}} \mathrm{Se}$ favored the improvement of electrochemical properties of the $\mathrm{CE}$ as well as the photovoltaic performance of the solar cells. ${ }^{357}$ A PCE of $8.72 \%$ was obtained with this $\mathrm{Cu}_{\mathrm{x}} \mathrm{Se} / \mathrm{FTO} \mathrm{CE}$ and the cell showed good stability over $168 \mathrm{~h}$.

Apart from copper chalcogenides based CEs, other binary or alloyed metal chalcogenides, such as $\mathrm{PbS},{ }^{358-362} \mathrm{CoS},{ }^{363-366} \mathrm{FeS},{ }^{367-370} \mathrm{NiS},{ }^{371,372}$ and $\mathrm{Cu}_{2} \mathrm{ZnSnS}_{4}$ (CZTS) or $\mathrm{Cu}_{2} \mathrm{ZnSnSe}_{4}$ $(\mathrm{CZTSe})^{373-379}$ etc. have also been used to fabricate CE in QDSCs. For example, Zaban and coworkers used $\mathrm{PbS}$ as $\mathrm{CE}$ catalytic materials in QDSCs. It was demonstrated that the PbS CE had higher catalytic activity towards polysulfide electrolyte compared to Pt CE. ${ }^{362}$ Teng and coworkers applied a photoactive p-type PbS film as CE to construct QDSCs. The authors proposed that the p-type characteristics of the $\mathrm{PbS}$ film forms a partial tandem junction between the $\mathrm{PbS}$ and the anode, resulting in an increase in the $V_{\mathrm{oc}}$ and the FF values, achieving a PCE of 4.7\%. ${ }^{359}$ Jin and coworkers reported a CE based on $\mathrm{CoS}_{2}$ thin film on borosilicate glass prepared by thermal sulfidation. The $\mathrm{CoS}_{2}$ film exhibited high catalytic activity towards polysulfide electrolyte and good reproducibility and stability in polysulfide electrolyte, giving a PCE of $4.16 \%{ }^{365}$ Another kind of metal chalcogenide that has been widely investigated as CE catalytic material is quaternary CZTS or CZTSe, which are also famous light harvesting materials in thin film solar cells. Lee and coworkers applied the CZTS microspheres-coated FTO substrate as a CE in QDSCs and achieved a PCE of $3.73 \% .{ }^{379}$ It was shown that the catalytic activity of CZTS towards $\mathrm{S}^{2-} / \mathrm{S}_{\mathrm{n}}{ }^{2-}$ redox couple was distinctly higher than the conventional Pt CE. Cheng et al. prepared porous structured CZTSe CE by spray deposition method, achieving a PCE of $4.35 \%{ }^{380}$ 
4.3.3. Carbon materials. Carbon materials are considered as attractive catalysts due to its advantages of low cost, high durability, and good conductivity. Thus, carbon materials are potential candidates for the construction of CEs in DSCs and QDSCs. As early as 1996, Grätzel and coworkers first explored a graphitic-carbon black mixture as CE material in DSCs and obtained a PCE of $6.67 \% .{ }^{381}$ In fact, various carbon materials have been explored as CEs in QDSCs. ${ }^{103,104,382-390}$ For example, Meng et al. deposited a mixture of activated carbon and conductive carbon black on FTO substrate as CE, exhibiting a PCE of $1.47 \%$ for CdS QDSCs. ${ }^{390} \mathrm{Ko}$ and $\mathrm{Yu}$ and coworkers have devoted much work to carbon materials based CEs in QDSCs. For example, Ko and coworkers employed hierarchical nanostructured spherical carbon with hollow core/mesoporous shell (HCMS) to prepare CE in QDSCs. It was found that the charge transfer resistance $\left(R_{\mathrm{ct}}\right)$ at the interface of the $\mathrm{CE} /$ polysulfide electrolyte was decreased in comparison with Pt CE, delivering a PCE of 3.90\% with use of CdSe QDs as sensitizer. ${ }^{383}$ Furthermore, they explored ordered multimodal porous carbon (OMPC) as CE material in both DSCs and QDSCs. The OMPC possesses large surface area and well-developed three-dimensional (3D) interconnected ordered macropore framework with open mesopores embedded in the macropore walls, exhibiting high catalytic activities and fast mass transfer kinetics toward both $\mathrm{I}^{-} / \mathrm{I}_{3}{ }^{-}$and $\mathrm{S}^{2-} / \mathrm{S}_{\mathrm{n}}{ }^{2-}$ redox couples. As a result, an impressive PCE of $4.36 \%$ was obtained based on CdSe QDs in $2010 .{ }^{103}$ Besides, they also developed mesoporous carbon nanofibers (MCNFs) based CE in QDSCs, showing a PCE of $4.81 \% .^{104}$
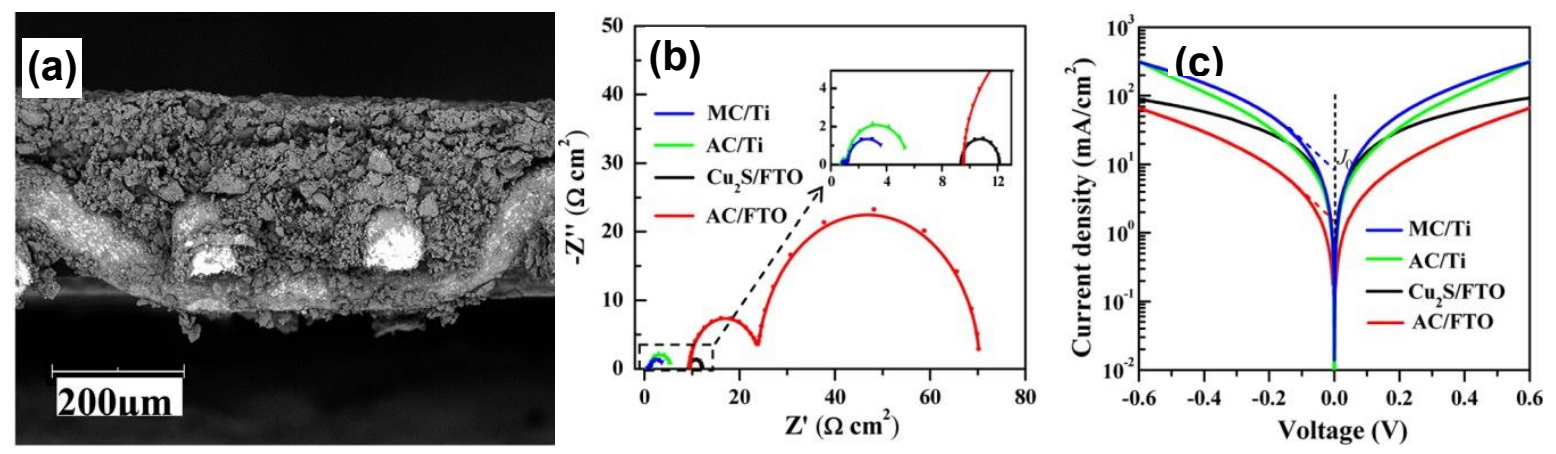

Fig. 24 (a) SEM image of cross section of MC/Ti CE. (b) Nyquist plots and (c) Tafel polarization curves of symmetric cells based on identical CEs. Reprinted with permission from ref. 118. Copyright (2016) American Chemical Society. 
Table 4 Summary of the representative photovoltaic performance for QDSCs based on different CEs.

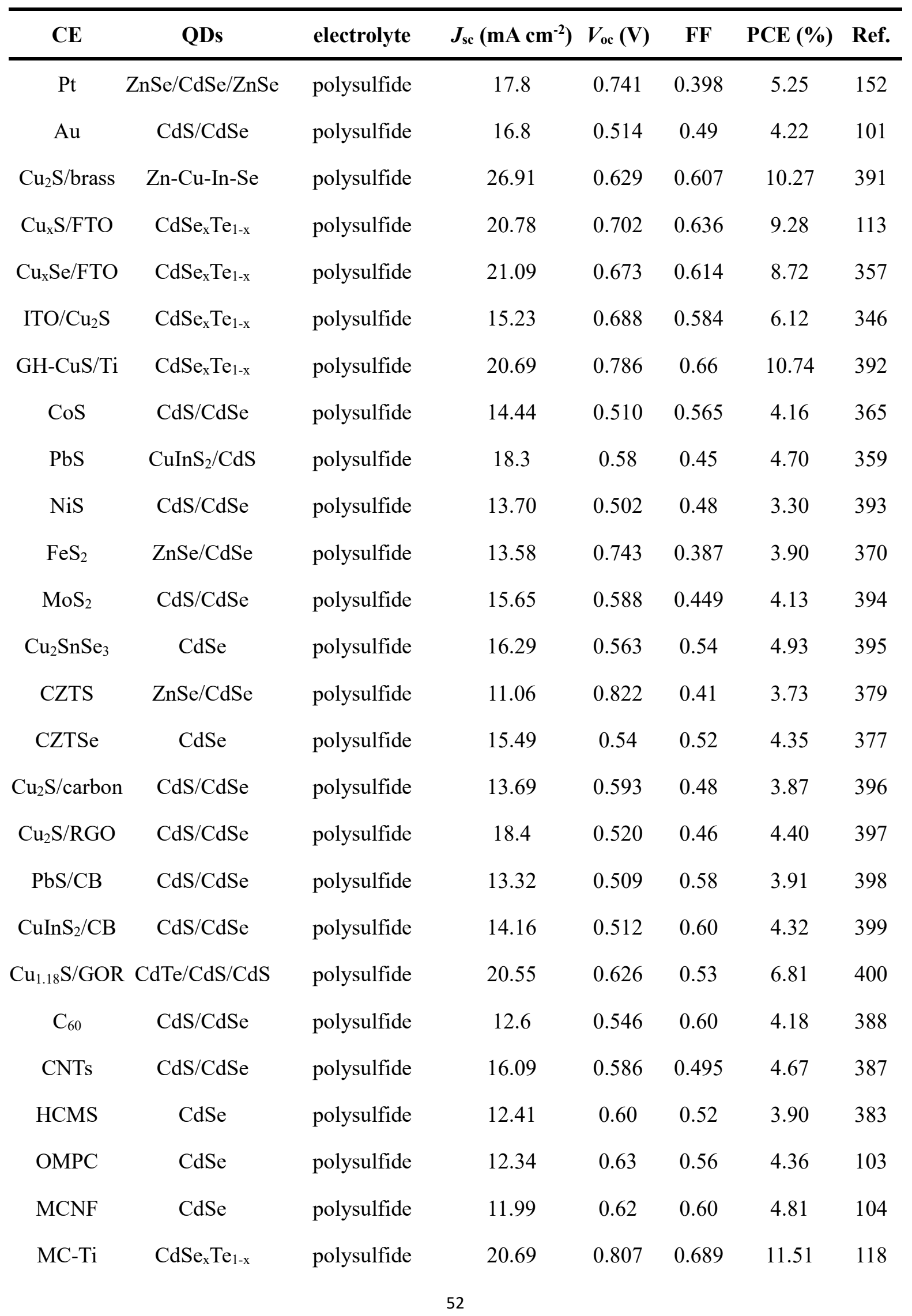




\begin{tabular}{llllllll} 
N-MC-Ti & Zn-Cu-In-Se & polysulfide & 25.67 & 0.759 & 0.639 & 12.45 & 119 \\
\hline
\end{tabular}

Even though various carbon materials have been explored as CEs in QDSCs, the obtained solar cell performance were still unsatisfactory compared to the conventional $\mathrm{Cu}_{2} \mathrm{~S} \mathrm{CE}$ based one. This can be attributed to the limited catalytic activity, internal resistance, and stability of the carbon film based CEs. Accordingly, Zhong's group developed Ti mesh supported carbon film as $\mathrm{CE}$ to improve the catalytic activity, conductivity and mechanical stability of CEs. ${ }^{118,119}$ For example, as shown in Fig. 24, they used mesoporous carbon (MC) to fabricate Ti mesh supported MC (MC/Ti) CE in QDSCs. It was found that the MC/Ti CE presented superior performance compared to the state-of-art $\mathrm{Cu}_{2} \mathrm{~S} / \mathrm{FTO} \mathrm{CE}$. On one hand, the excellent performance of this $\mathrm{MC} / \mathrm{Ti} \mathrm{CE}$ can be attributed to the robust carbon film with good conductivity and submillimeter thickness due to the confined area in Ti mesh substrate, ensuring high catalytic capacity. On the other hand, the 3D interconnected mesoporous framework structure of MC realizes fast electrolyte mass transfer kinetics. Finally, a PCE up to $11.51 \%$ (11.16\% for certified value) was obtained, nearly a $30 \%$ improvement relative to the $\mathrm{Cu}_{2} \mathrm{~S} / \mathrm{FTO} \mathrm{CE}$ based one. ${ }^{118}$ Furthermore, they also explored nitrogen-doped mesoporous carbon (N-MC) materials to prepare CE in QDSCs. The incorporation of nitrogen heteroatoms into $\mathrm{MC}$ can further improve its catalytic activity towards polysulfide electrolyte, yielding a PCE over $12 \% .{ }^{119}$ It is noted that the exploration of carbon materials based effective CEs dramatically promoted the development of QDSCs in recent years.

4.3.4. Composite CEs. Usually, the performance of CE is limited by the unsatisfactory function of single component material. Therefore, an efficient way to overcome the shortcoming of single materials based CEs is to fabricate composite CEs with use of multi-component materials. ${ }^{329,397-412}$ For example, Kamat and coworkers designed a reduced graphene oxide (RGO)- $\mathrm{Cu}_{2} \mathrm{~S}$ composite $\mathrm{CE} .^{397}$ The 2-D structure of RGO facilitates the shuttling of electrons and provides high surface area for the formation of $\mathrm{Cu}_{2} \mathrm{~S}$ reactive sites. As a result, an impressive PCE of 4.4\% was obtained based on CdS/CdSe QDSCs. Meng and coworkers prepared PbS/carbon black composite CEs on FTO substrate. ${ }^{398}$ The nanosized $\mathrm{PbS}$ provided large area of catalytic sites and the carbon black improved both the mechanical strength and electrical conductivity of the $\mathrm{CE}$. The constructed cell based on this composite CE exhibited a PCE of 3.91\% with use of CdS/CdSe QDs sensitizer. What's more, the cell showed outstanding stability over $1000 \mathrm{~h}$ under room conditions without degradation. They 
also prepared $\mathrm{CuInS}_{2}$ /carbon composite $\mathrm{CE}$, obtaining a higher PCE of $4.32 \%$ and good stability over 1000 h. ${ }^{399}$ Choi and coworkers prepared carbon dot-Au nanoraspberries CE, exhibiting high electrocatalytic activity, lower charger-transfer resistance, and larger exchange current density than the reference $\mathrm{Au} \mathrm{CE}$ and obtained a PCE of $5.4 \%{ }^{329}$ Bhattacharyya and coworkers reported a composite CE containing microwave synthesized $\mathrm{Cu}_{\mathrm{x}} \mathrm{S}$ and graphene oxide nanoribbon (GOR) for QDSCs. ${ }^{400}$ The composite CE exhibited higher electrocatalytic activity towards polysulfide electrolyte benefitting from the presence of organic functional groups, graphitic edge sites and a quasi-one-dimensional structure of the GOR. Finally, PCEs of $5.42 \%$ and $6.81 \%$ was obtained for $\mathrm{CdS} / \mathrm{CdSe}$ and $\mathrm{CdTe} / \mathrm{CdS} / \mathrm{CdS}$ dual sensitized QDSCs, respectively.

\subsection{Electrolyte or hole transporting materials}

Electrolyte redox couple or hole-transporting material (HTM) is also one of the most crucial components in QDSCs, serving to regenerate QDs and shuttle the photo-generated holes to the CE. ${ }^{6,51}$ To evaluate a redox couple or HTM in QDSCs, the following terms should be considered: (1) low corrosivity to the QDs to maintain the good long term stability of the cell device; (2) appropriate redox potential to regenerate QDs effectively and keep a high $V_{\text {oc }}$ value meanwhile; (3) high ion conductivity to facilitate hole transfer; (4) good stability of itself and high transparency in the visible light window; (5) fully regenerative, avoiding the use of non-regenerative hole scavengers. Generally, electrolyte or HTM can significantly influence the solar cell performance and long-term stability of the device. According to the apparent characteristics, electrolyte or HTM in QDSCs can be summarized into three kinds: liquid, quasi-solid-state, and solid-state based ones. The representative photovoltaic performances for QDSCs based on different electrolytes or HTMs are summarized in Table 5.

4.4.1. Liquid electrolyte. Liquid electrolyte is composed of a redox couple. Even though the well-studied $\mathrm{I}^{-} / \mathrm{I}_{3}{ }^{-}$redox couple is a state-of-art electrolyte in DSCs, its high corrosivity to most QDs limits its application in QDSCs. Nevertheless, researchers found that sulfide/polysulfide redox couple $\left(\mathrm{S}^{2-} / \mathrm{S}_{\mathrm{n}}{ }^{2-}\right)$ was a suitable choice to stabilize most of the QDs. ${ }^{94,413,414}$ Therefore, polysulfide electrolyte is the most popular choice in QDSCs, and most of the obtained high PCEs are derived from this electrolyte based system. ${ }^{414}$ However, the unwanted disadvantages of polysulfide electrolyte limited the development of QDSCs: (i) the redox potential of polysulfide electrolyte is relatively high, leading to the energy lose and consequently low $V_{\mathrm{oc}}$; (ii) the QDs regeneration rate is slow, leading to the accumulation of 
holes in QDs and therefore decreasing the QDs stability and increasing the recombination rate as well. ${ }^{32}$ Thus, considerable research efforts have been devoted to explore new kinds of redox couple, or modify polysulfide electrolyte with use of additives to improve its electrochemical properties.
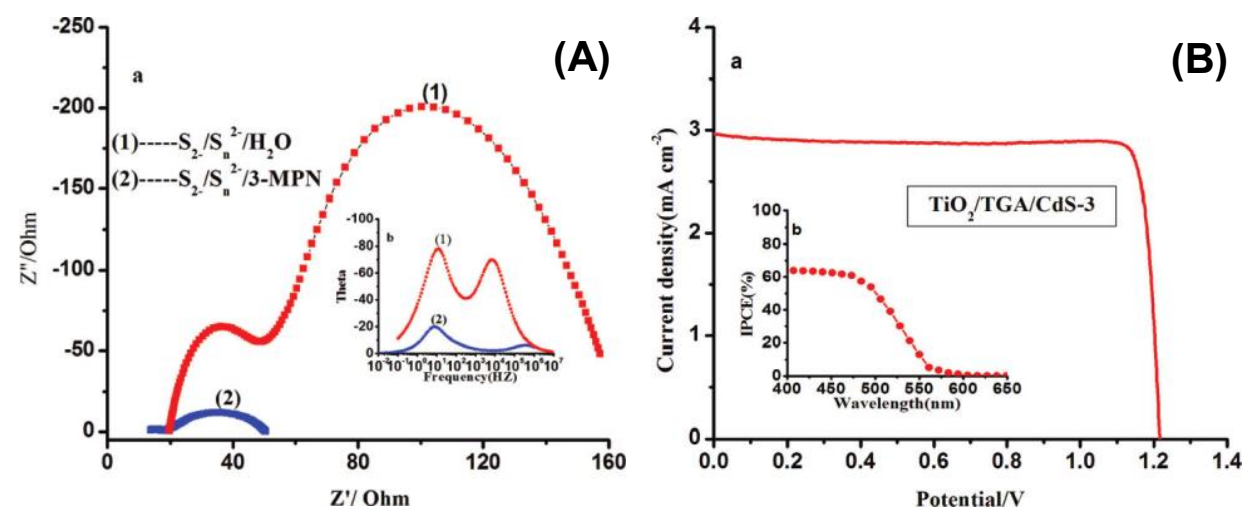

Fig. 25 (A) Electrochemical impedance spectra of (1) $0.5 \mathrm{M} \mathrm{Na}_{2} \mathrm{~S}+2.0 \mathrm{M} \mathrm{S}$ in $\mathrm{H}_{2} \mathrm{O}$ and (2) $\left[\left(\mathrm{CH}_{3}\right)_{4} \mathrm{~N}\right]_{2} \mathrm{~S} /\left[\left(\mathrm{CH}_{3}\right)_{4} \mathrm{~N}\right]_{2} \mathrm{~S}_{\mathrm{n}}$ in 3-methoxypropionitrile (MPN): (a) complex plane impedance plots; (b, inset) Bode phase plots. (B) $J-V$ characteristics of the $\mathrm{TiO}_{2} / \mathrm{TGA} / \mathrm{CdS}-3$-based cell measured under one-sun illumination (a) and IPCE spectrum (b, inset) of the cell based on the $\mathrm{TiO}_{2} / \mathrm{TGA} / \mathrm{CdS}-3$ electrode. Reprinted with permission from ref. 415. Copyright (2011) American Chemical Society.

Much work so far has focused on exploration of new kinds of redox couples in QDSCs. ${ }^{102,415-418}$ One of them is cobalt complex based electrolyte, which is also an efficient electrolyte in DSCs. ${ }^{102,320,325,419}$ For example, Grätzel and coworkers introduce cobalt redox couple of $\left[\mathrm{Co}(\mathrm{o}-\right.$ phen $\left.) 3^{2+/ 3+}\right]$ as electrolyte in QDSCs. ${ }^{102}$ The cell with use of CdSeTe QDs sensitizers showed a PCE of $4.18 \%$ at 0.1 sun illumination. Ning and coworkers developed a tetramethylthiourea (TMTU) based organic electrolyte for QDSCs. ${ }^{416}$ It was found that the charge transfer resistance at electrolyte/CE interface was distinctly smaller for the cell based on this organic electrolyte than the polysulfide electrolyte based one. Sun and coworkers employed an organic polysulfide redox couple, $\left[\left(\mathrm{CH}_{3}\right)_{4} \mathrm{~N}\right]_{2} \mathrm{~S} /\left[\left(\mathrm{CH}_{3}\right)_{4} \mathrm{~N}\right]_{2} \mathrm{~S}_{\mathrm{n}}$, as electrolyte in CdS QDSCs. ${ }^{415}$ The oxidation potential of this organic polysulfide redox couple is $1.045 \mathrm{~V} v \mathrm{~s}$ NHE, which is about $0.5 \mathrm{~V}$ lower than that of the $\mathrm{S}^{2-} / \mathrm{S}_{\mathrm{n}}{ }^{2-}$ redox couple, exhibiting a $V_{\text {oc }}$ as high as $1.2 \mathrm{~V}$ and a FF of 0.89 (Fig. 25). Mora-Seró and coworkers designed a novel pyrrolidinium-based ionic liquid as electrolyte in QDSCs. ${ }^{417}$ The CdSe QDSCs based on this ionic liquid electrolyte reached a PCE of $1.86 \%$, with good stability over 10 days. Tachibana 
and coworkers employed ferricyanide/ferrocyanide $\left(\mathrm{Fe}(\mathrm{CN}) 6^{3-/ 4-}\right)$ redox couple, which has a relatively positive redox potential (around $0.25 \mathrm{~V} v s \mathrm{Ag} / \mathrm{AgCl}$ ), as electrolyte for QDSCs. This electrolyte contributed to an enhancement of $V_{\mathrm{oc}}$ to $0.8 \mathrm{~V}^{418}$

However, according to the above mentioned works, even though the application of novel redox couples bring forward an obvious improvement in $V_{\text {oc }}$ values, the PCEs still lag far from the polysulfide electrolyte based ones. This may be partially due to the limited stability of QDs in the electrolyte or the slow QDs regeneration rate. Thus, it remains challenging to develop efficient redox couple to boost the PCE of QDSCs beyond that based on the state-of-the-art polysulfide electrolyte. This is an important challenge for QDSCs in order to further increase their performance in the future.

Table 5 Summary of the representative photovoltaic performance for QDSCs based on different redox couples and solid-state HTMs.

\begin{tabular}{|c|c|c|c|c|c|c|c|}
\hline redox couple or HTMs & QDs & $\mathbf{C E}$ & $\left.J_{\mathrm{sc}}(\mathrm{mA} \mathrm{cm})^{-2}\right)$ & $V_{\text {oc }}(\mathrm{V})$ & FF & PCE (\%) & Ref. \\
\hline polysulfide & ZCISe-CdSe & $\mathrm{MC} / \mathrm{Ti}$ & 27.39 & 0.752 & 0.619 & 12.75 & 112 \\
\hline$\left[\left(\mathrm{CH}_{3}\right)_{4} \mathrm{~N}\right]_{2} \mathrm{~S} /\left[\left(\mathrm{CH}_{3}\right)_{4} \mathrm{~N}\right]_{2} \mathrm{~S}_{\mathrm{n}}$ & $\mathrm{CdS}$ & $\mathrm{Pt}$ & 3.0 & 1.2 & 0.89 & 3.2 & 415 \\
\hline polysulfide-IL & $\mathrm{CdSe}$ & $\mathrm{Pt}$ & 13.85 & 0.42 & 0.32 & 1.86 & 417 \\
\hline $\mathrm{Fe}(\mathrm{CN})_{6}^{3-} /^{4-}$ & $\mathrm{CdS}$ & $\mathrm{Pt}$ & 3.8 & 0.8 & 0.66 & 2.0 & 418 \\
\hline$\left[\mathrm{Co}(\mathrm{bpy})_{3}\right]^{2+/ 3+}$ & $\mathrm{CdS}$ & $\mathrm{Pt}$ & 2.34 & 0.704 & 0.62 & 1.01 & 325 \\
\hline$\left[\mathrm{Co}(\mathrm{bpy})_{3}\right]^{2+/ 3+}$ & $\mathrm{Au}_{\mathrm{x}}$ & $\mathrm{Pt}$ & 3.96 & 0.832 & 0.716 & 2.36 & 211 \\
\hline$\left[\mathrm{Co}(\mathrm{o}-\mathrm{phen})_{3}\right]^{2+/ 3+}$ & $\mathrm{CdSe}_{\mathrm{x}} \mathrm{Te}_{1-\mathrm{x}}$ & $\mathrm{Pt}$ & 4.94 & 0.67 & 0.54 & 1.77 & 102 \\
\hline tetramethylthiourea & $\mathrm{ZnSe} / \mathrm{CdS}$ & $\mathrm{Pt}$ & 2.25 & 0.66 & 0.58 & 0.86 & 416 \\
\hline$[\mathrm{DHexBIm}][\mathrm{SCN}]^{\mathrm{a}}$ & $\mathrm{CdS} / \mathrm{CdSe}$ & $\mathrm{PbSe}$ & 12.58 & 0.60 & 0.56 & 4.26 & 420 \\
\hline $\mathrm{CuSCN}$ & $\mathrm{CdSe}$ & $\mathrm{Au}$ & 4.00 & $\sim 0.50$ & - & 2.3 & 421 \\
\hline spiro-OMeTAD ${ }^{\mathrm{b}}$ & $\mathrm{PbS}$ & $\mathrm{Au}$ & 13.56 & 0.52 & 0.579 & 4.10 & 133 \\
\hline $\mathrm{P} 3 \mathrm{HT}^{\mathrm{c}}$ & $\mathrm{PbS} / \mathrm{CuS}$ & $\mathrm{Au}$ & 20.7 & 0.60 & 0.65 & 8.07 & 422 \\
\hline PCPDTBT $^{d}$ & $\mathrm{Sb}_{2} \mathrm{~S}_{3}$ & $\mathrm{Au}$ & 15.3 & 0.616 & 0.657 & 6.18 & 423 \\
\hline
\end{tabular}

a1,3-dihexylbenzimidazolium cation combined with the SCN anions;

b2,2',7,7'-tetrakis-(N,N-di-p methoxyphenylamine)9,9'-spirobifluorene; cpoly(3-hexylthiophene);

${ }^{d} \operatorname{poly}(2,6-(4,4-b i s-(2-e t h y l h e x y l)-4 H$-cyclopenta[2,1-b;3,4-b']dithiophene)-alt-4,7(2,1,3-benzothiadiazole)) 
Apart from searching for new redox couples, the modification of polysulfide electrolyte with use of additives to improve its electrochemical properties is another way to improve the performance of QDSCs. This strategy has been intensively investigated in DSCs, and various additives, such as 4-tert-butylpyridine (TBP), lithium ions, and guanidinium thiocyanate $(\mathrm{GuSCN})$, have been explored to modify $\mathrm{I}^{-} / \mathrm{I}_{3}{ }^{-}$electrolyte. ${ }^{6}$ It has been demonstrated that these additives can distinctly contribute to an improvement in photovoltaic performance by either inhibiting charge recombination at the photoanode/electrolyte interface, or shifting the conduction band edge of $\mathrm{TiO}_{2}$. What's more, the redox potential of the redox couple can also be changed, thus influencing the $V_{\text {oc }}$ of cells. Recently, some initial attempts have been made to modify polysulfide electrolyte with use of additives in QDSCs. ${ }^{205,391,424,425}$ For example, Zhong's group adopted water-soluble polymers, including poly(ethylene glycol) (PEG) and poly(vinyl pyrrolidone) (PVP) as additives in polysulfide electrolyte and remarkable enhancement in PCE was observed, especially in the $V_{\mathrm{oc}}$ and FF values. ${ }^{205,424}$ Meng's group reported a fumed $\mathrm{SiO}_{2}$ nanoparticles modified polysulfide electrolyte. ${ }^{425}$ It is revealed that the addition of $\mathrm{SiO}_{2}$ nanoparticles in the electrolyte can create an energy barrier for the recombination at QDs/electrolyte as well as $\mathrm{TiO}_{2} /$ electrolytethe interfaces. Finally, a PCE of $11.23 \%$ was achieved, $28.6 \%$ higher than the reference cell. Zhong and coworkers employed tetraethyl orthosilicate (TEOS) as additive in polysulfide electrolyte and investigated its effect on charge transfer dynamics at photoanode/electrolyte interface. ${ }^{391}$ The PCE of the corresponding cell can be improved from $11.75 \%$ to $12.34 \%$ with the aid of TEOS addition, arising from the reduction of recombination loss. 

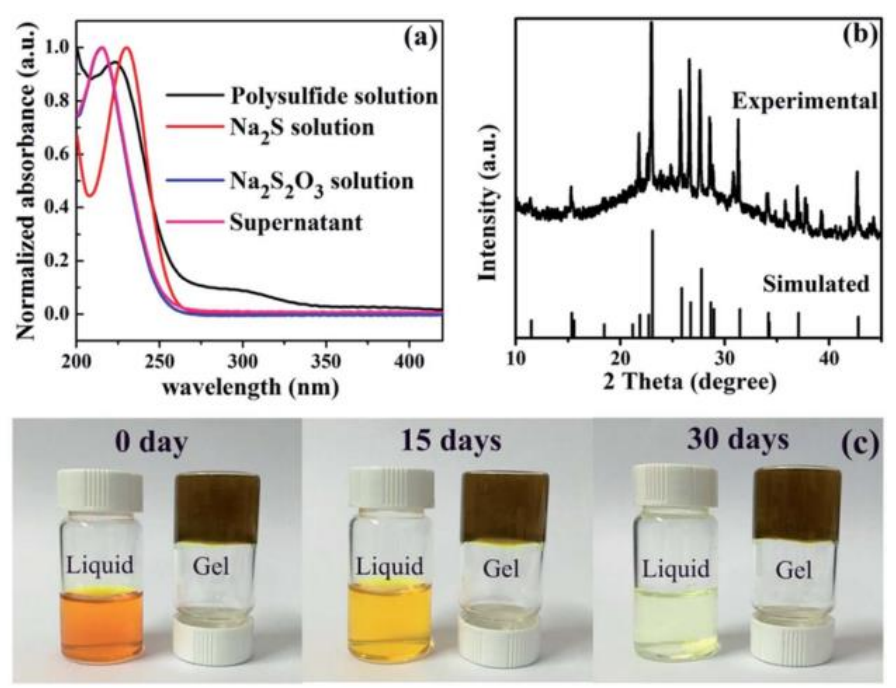

Fig. 26 (a) UV-Vis spectra of the polysulfide electrolyte, $\mathrm{Na}_{2} \mathrm{~S}, \mathrm{Na}_{2} \mathrm{~S}_{2} \mathrm{O}_{3}$ solution and the supernatant. (b) Experimental powder X-ray diffraction (PXRD) spectrum of the precipitates in the liquid polysulfide electrolyte solution and simulated PXRD spectrum of b-sulfur. (c) The color change of the electrolytes during 30 days. Reprinted with permission from ref. 426. Copyright (2016) Royal Society of Chemistry.

4.4.2. Quasi-solid-state electrolyte. The drawbacks of easy leakage and volatilization for liquid electrolytes bring about the poor stability of cell device and so limit its application. The fabrication of quasi-solid-state QDSCs employing gel electrolyte is a promising way to obtain high stable QDSCs. ${ }^{426-432}$ Meng and coworkers reported for the first time a quasi-solid-state polysulfide electrolyte with use of polyacrylamide-based hydrogel as the polymer matrix and obtained a PCE of $4.0 \% .{ }^{427}$ It was demonstrated that the 3-D continuous porous network of the polymer is very effective to enhance the absorbent ability toward the liquid electrolyte and ion transportation capability. The gel electrolyte exhibits a high ionic conductivity of $0.093 \mathrm{~S} \cdot \mathrm{cm}^{-1}$, which is very close to that of the liquid polysulfide electrolyte $\left(0.104 \mathrm{~S} \cdot \mathrm{cm}^{-1}\right)$. Kuang and coworkers prepared a gel polysulfide electrolyte using dextran as gelator. ${ }^{429}$ This gel electrolyte has a similar conductivity with the liquid one through the optimization of the gelator concentration and the corresponding QDSCs exhibit a PCE of 3.23\%. Meng and coworkers applied natural polysaccharide konjac glucomannan (KGM) as the polymer matrix to prepare gel electrolyte and the electrolyte and $\mathrm{Cu}_{2} \mathrm{~S}$ counter electrode are prepared in one step without mold. ${ }^{428}$ The QDSCs based on this gel electrolyte showed a comparable PCE $(4.06 \%)$ with the liquid electrolyte based one $(4.22 \%)$ while the cell stability was remarkably improved. Tang and coworkers synthesized graphene implanted polyacrylamide (PAAm-G) 
conducting gel electrolytes for quasi-solid-state QDSCs. ${ }^{430}$ They utilized osmotic press across the PAAm-G and capillary force within the three-dimensional micropores as driving forces to improve the dosage of $\mathrm{S}^{2-} / \mathrm{S}_{\mathrm{n}}{ }^{2-}$ redox couple and obtained a PCE of $2.34 \%$ based on CdS QD sensitizer. Huo and coworkers employed 12-hydroxystearic acid as a low molecular mass organogelator to gelate the polysulfide electrolyte and also found an improvement in the cell stability. ${ }^{431}$ Zhong's group used sodium polyacrylate (PAAS) and sodium carboxymethylcellulose (CMC-Na) as gelators to prepare quasi-solid-state gel polysulfide electrolytes for QDSCs (Fig. 26), and both of the gel electrolytes contributed to an improvement of the cell stability under continuous irradiation for 90 h. ${ }^{426,432}$

4.4.3. All solid-state QDSCs. The all solid-state device can thoroughly overcome the drawbacks of leakage and volatilization of liquid electrolyte, owning the potential to realize the long-term stability of the constructed QDSC. ${ }^{6}$ The architecture of all solid-state QDSCs is a little different from the liquid-junction QDSCs. ${ }^{433}$ Typically, a solid-state hole-transporting material (HTM) instead of liquid redox couple is used as the hole transport medium and an evaporated back electrode instead of a counter electrode is applied to collect carriers.
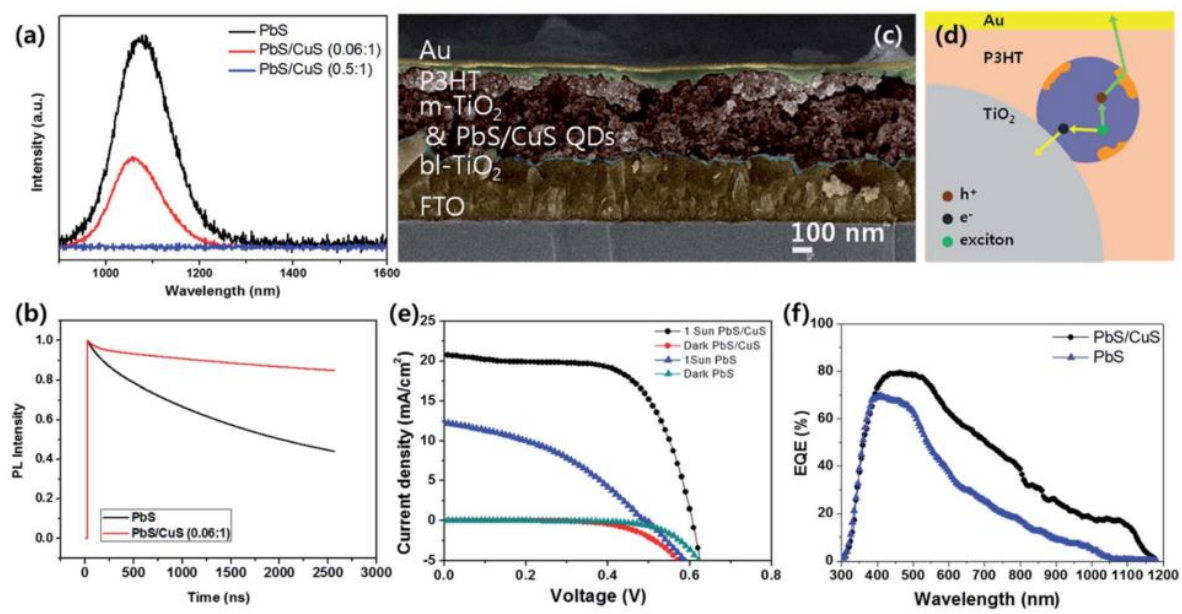

Fig. 27 (a) Static PL spectra of PbS, PbS[CuS] and CuS. (b) Luminescence decays (TCSPC) of pristine $\mathrm{PbS}$ solution (black) and $\mathrm{PbS}[\mathrm{CuS}]$ solution (red). (c) SEM cross-sectional images of QD-SSCs, (d) illustration of the proposed working mechanism, generated excitons by light illumination are rapidly separated into free charge carriers. (e) $I-V$ curve of pristine $\mathrm{PbS}$ QD-SSCs (blue, dark cyan) and PbS[CuS] QD-SSCs (black, red), (f) EQE spectra of pristine $\mathrm{PbS}$ QD-SSCs (blue) and PbS[CuS] QD-SSCs. Reprinted with permission from ref. 422. Copyright (2016) Royal Society of Chemistry. 
For solid-state QDSCs, PbS QD is one of the commonly used sensitizer.97,133,251,422,434,435 The first report about all solid-state QDSCs is by Grätzel and coworkers, in which they employed spiro-OMeTAD as the HTM and obtained an efficiency of $0.49 \%$ under 0.1 sun illumination with use of PbS QDs. ${ }^{97}$ Afterwards, they improved the PCE to $1.46 \%$ through the optimization of the pore size of the $\mathrm{TiO}_{2}$ films and the $\mathrm{PbS}$ deposition cycles. ${ }^{251}$ Seok and coworkers deposited multiply layered $\mathrm{PbS}$ QDs on $\mathrm{TiO}_{2}$ electrode and fabricated solid-state QDSCs with use of poly(3-hexylthiophene) (P3HT) as HTM. ${ }^{434}$ The cell performance was further improved by a post-EDT treatment, achieving a PCE of $2.9 \%$ under 1 sun illumination. Kim and coworkers synthesized CuS-embedded PbS QDs ( $\mathrm{PbS} / \mathrm{CuS}$ ) by cation exchange reaction and applied them as sensitizer in solid-state QDSCs (Fig. 27). ${ }^{422}$ It was shown that the introduction of $\mathrm{CuS}$ around $\mathrm{PbS}$ can prolong the exciton lifetime and increase the absorption due to the surface plasmon effect. As a result, a PCE of $8.07 \%$ was obtained with architecture of $\mathrm{TiO}_{2} / \mathrm{PbS}-\mathrm{CuS} / \mathrm{P} 3 \mathrm{HT} / \mathrm{Au}$.

II - VI group CdS or CdSe QDs were also used to fabricate solid-state QDSCs. ${ }^{421,436-440}$ For example, in 2005, Hodes and coworkers sensitized $\mathrm{ZnO}$ nanowires with CdSe and fabricated solid-state QDSCs with use of CUSCN as HTM, yielding a PCE of 2.3\% under 360 $\mathrm{W} / \mathrm{m}^{2}{ }^{2}{ }^{421}$ Larramona and coworkers used CdS QD sensitized $\mathrm{TiO}_{2}$ porous film to construct solid-state QDSCs based on CuSCN HTM, obtaining a PCE of $1.3 \%$ at 1 sun. ${ }^{436}$ Bach and coworkers applied diisooctyl phosphonic acid (DIOPA) and benzenethiol (BT) derivatives to modify the surface of $\mathrm{CdS} / \mathrm{CdSe}$ sensitized $\mathrm{TiO}_{2}$ electrode and obtained a PCE of $0.88 \%$, which is higher than the reference cell $(0.65 \%) .{ }^{437}$ Song and coworkers employed P3HT acting as both HTM and assistant light absorber in CdS solid-state QDSCs, exhibiting a PCE of $1.42 \%{ }^{438}$ Lianos and coworkers demonstrated that the combination of $\mathrm{ZnSe}$ in CdSe QDs was beneficial for the improvement of photovoltaic performance of the corresponding solid-state QDSCs, arising from the more favorable arrangement of the energy levels. ${ }^{439}$ What's more, it was found that the addition of a small quantity of $\mathrm{Na}_{2} \mathrm{~S}$ in P3HT HTM also contributed to an improved PCE of $3.4 \%$. 

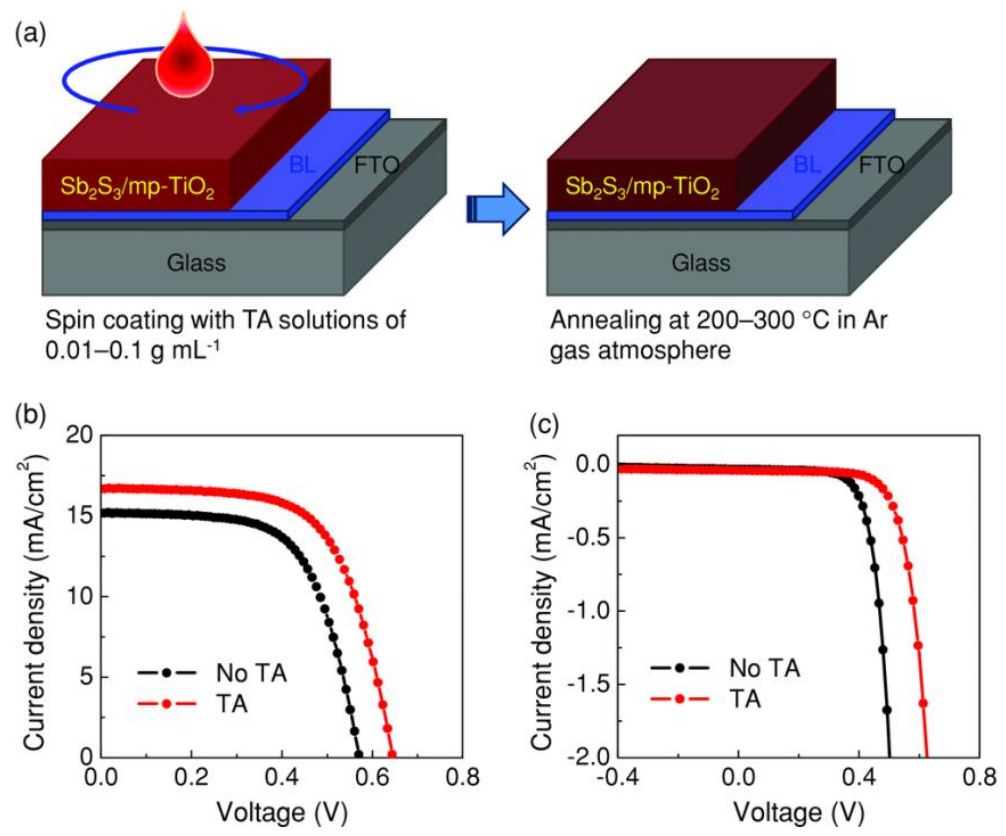

Fig. 28 Thioacetamide (TA) surface sulfurization and its effects on photovoltaic performance. (a) Schematic diagram for the TA surface sulfurization process in the $\mathrm{Sb}_{2} \mathrm{~S}_{3}$-sensitized inorganic-organic heterojunction solar cells. (b) Effects of TA sulfurization on the $J-V$ characteristics measured under AM $1.5 \mathrm{G}$ solar irradiance $\left(100 \mathrm{~mW} \mathrm{~cm}{ }^{-2}\right)$ and (c) corresponding dark $J-V$ curves. In $(\mathrm{b}, \mathrm{c})$, the devices with and without TA treatment are denoted as TA and No TA, respectively. Reprinted with permission from ref. 208. Copyright (2014) Wiley.

$\mathrm{Sb}_{2} \mathrm{~S}_{3}$ is another promising light harvesting material in the construction of all solid-state QDSCs due to its suitable band gap $(1.7 \mathrm{eV})$, strong absorption coefficient $\left(1.8 \times 10^{5} \mathrm{~cm}^{-1}\right)$, and environmental friendly characteristics. ${ }^{94,208,423,441-455}$ Initially, $\mathrm{Sb}_{2} \mathrm{~S}_{3}$ was used as light harvesting material in liquid-junction cells. ${ }^{94}$ However, it was found that the stability of $\mathrm{Sb}_{2} \mathrm{~S}_{3}$ in liquid electrolyte was very poor. The first report about all solid-state $\mathrm{Sb}_{2} \mathrm{~S}_{3}$ QDSCs was presented by Hodes's group. ${ }^{441}$ They deposited $\mathrm{Sb}_{2} \mathrm{~S}_{3}$ on $\mathrm{TiO}_{2}$ mesoporous film through CBD method and a PCE of $3.37 \%$ was obtained with use of CuSCN as HTM. The cell also showed good stability over 3 days of illumination under load. Afterwards, Hodes and Grätzel and coworkers employed spiro-MeOTAD instead of $\mathrm{CuSCN}$ as HTM, delivering PCEs of 5.2\% and $3.1 \%$ at 0.1 and 1 sun irradiation, respectively. ${ }^{443}$ Seok and Grätzel and coworkers found that P3HT is a suitable HTM in solid-state $\mathrm{Sb}_{2} \mathrm{~S}_{3}$ QDSCs. ${ }^{442}$ The P3HT in the device can act as both a hole conductor and light harvesting material, achieving a IPCE of $80 \%$ and a PCE of $5.13 \%$ under 1 sun illumination. Since then, the $\mathrm{Sb}_{2} \mathrm{~S}_{3}$ attracted much attention as light 
harvesting material to fabricate all solid-state QDSCs since the obtained PCE is comparable to solid-state DSCs at that time. ${ }^{6}$

Seok's group has devoted much effort to improve the PCE of $\mathrm{Sb}_{2} \mathrm{~S}_{3}$ or $\mathrm{Sb}_{2} \mathrm{Se}_{3}$ based solid-state QDSCs through the optimization of the HTM and the light harvesting material layer. For example, they investigated the effect of several HTMs on the cell performance of solid-state $\mathrm{Sb}_{2} \mathrm{~S}_{3}$ QDSCs, including P3HT, PCPDTBT, PCDTBT, and PTAA. ${ }^{423}$ It was found that the cell performance was strongly dependent on the chelation of thiophene moieties in HTMs toward $\mathrm{Sb}_{2} \mathrm{~S}_{3}$. Finally, the cell based on PCPDTBT HTM gave a best PCE of $6.18 \%$ under 1 sun illumination. To overcome the filter effect caused by absorption of P3HT in the visible light window, Seok and coworkers constructed a PCBM electron channel that can bridge mp- $\mathrm{TiO}_{2}$ and $\mathrm{P} 3 \mathrm{HT}$ to transfer the generated charge carriers in $\mathrm{P} 3 \mathrm{HT}$ to mp- $\mathrm{TiO}_{2}{ }^{445}$ Moreover, PCPDTBT was used in the device as an additional low band gap hole conducting polymer that can absorb light in a near-infrared region. As a result, the $J_{\mathrm{sc}}$ can be improved to $16.0 \mathrm{~mA} \mathrm{~cm} \mathrm{~cm}^{-2}$ and a PCE of $6.3 \%$ was obtained. Afterwards, Seok et al. introduced the surface sulfurization of $\mathrm{Sb}_{2} \mathrm{~S}_{3}$ by thioacetamide (TA) before the deposition of HTM layer (Fig. 28). ${ }^{208}$ It was found that the PCE of the cell can be significantly improved from $5.5 \%$ to $7.1 \%$ after the TA treatment. Deep-level transient spectroscopy (DLTS) revealed that the improved photovoltaic performance was mainly derived from the reduction of trap sites in $\mathrm{Sb}_{2} \mathrm{~S}_{3}$.

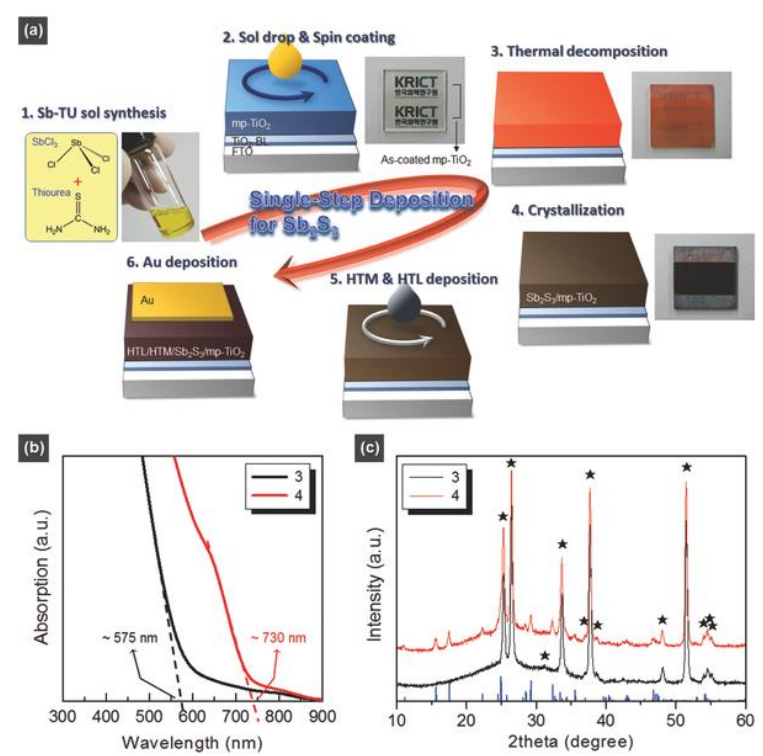

Fig. 29 (a) Schematic diagram of the fabrication process of our devices via $\mathrm{Sb}$-TU complex solution processing. (b) UV-vis absorption spectra and c) XRD patterns of the samples obtained after steps 3 and 4. Reprinted with permission from ref. 452. Copyright (2015) Wiley. 
The CBD method is generally used to deposit $\mathrm{Sb}_{2} \mathrm{~S}_{3}$ on $\mathrm{TiO}_{2}$ substrate. However, this approach is time-consuming and the experimental conditions should be precisely controlled (below $10{ }^{\circ} \mathrm{C}$ ) to avoid impurity phases. Gödel et al. reported a modified CBD approach to deposit $\mathrm{Sb}_{2} \mathrm{~S}_{3}$ under room temperature, showing a lower sub-bandgap trap state density and delivering a PCE of $5.1 \% .{ }^{454}$ Seok and coworkers developed a single-step process to deposit $\mathrm{Sb}_{2} \mathrm{~S}_{3}$ on $\mathrm{TiO}_{2}$ by spin-coating an $\mathrm{S} / \mathrm{Sb}$ ratio-controlled $\mathrm{SbCl}_{3}$-thiourea complex solution on the substrate (Fig. 29). ${ }^{452}$ This single-step approach was demonstrated to be efficient and reproducible to fabricate high quality $\mathrm{Sb}_{2} \mathrm{~S}_{3}$ layer and a comparable PCE of $6.4 \%$ was obtained relative to the conventional CBD method based one $(6.3 \%)$. Herein, it is noted that the size of $\mathrm{Sb}_{2} \mathrm{~S}_{3}$ obtained through this single-step approach was about $60 \mathrm{~nm}$, which is not in the range of quantum dot size. Thus, this kind of cell is usually named as semiconductor-sensitized solar cell (SSCs). ${ }^{433}$

Apart from $\mathrm{Sb}_{2} \mathrm{~S}_{3}, \mathrm{Sb}_{2} \mathrm{Se}_{3}$, which has a narrower band bap (1.1 eV), has also been applied as light harvesting material to fabricate solid-state sensitized solar cells. Seok and coworkers applied a single-step to deposit $\mathrm{Sb}_{2} \mathrm{Se}_{3}$ on mesoporous $\mathrm{TiO}_{2}$ by simple multiple cycles of spin-coating of a Se-SSP solution followed by thermal decomposition. ${ }^{456}$ The light absorption range can be expanded to $1050 \mathrm{~nm}$ due to the narrower band gap of $\mathrm{Sb}_{2} \mathrm{Se}_{3}$. The cell device exhibited a $J_{\text {sc }}$ of $22.3 \mathrm{~mA} \mathrm{~cm}^{-2}$, a $V_{\text {oc }}$ of $304.5 \mathrm{mV}$, a FF of $47.2 \%$, and a PCE of $3.21 \%$ under full one sun illumination. Besides, they also constructed $\mathrm{Sb}_{2}\left(\mathrm{~S}_{\mathrm{x}} / \mathrm{Se}_{1-\mathrm{x}}\right)_{3}$ graded-composition sensitizers based solid-state inorganic semiconductor-sensitized solar cells. ${ }^{450}$ They firstly deposited $\mathrm{Sb}_{2} \mathrm{Se}_{3}$ on $\mathrm{TiO}_{2}$ substrate by spin-coating of $\mathrm{Se}$ precursor and then $\mathrm{Sb}_{2} \mathrm{~S}_{3}$ was grown on the $\mathrm{Sb}_{2} \mathrm{Se}_{3}$ sensitized film through CBD method. The cell exhibited a best PCE of $6.6 \%$ with a high $J_{\mathrm{sc}}$ value of $24.9 \mathrm{~mA} \mathrm{~cm}^{-2}$.

In general, although all solid-state QDSC or SSC possess prominent advantages, especially their potential to realize long-term stability of the device, the obtained PCE is still low compared to the liquid-junction QDSCs. To date, the highest PCE for all solid-state QDSC or SSC is about $8 \%$. Materials are the main limitation for solid-state QDSC, including light harvesting materials as well as HTMs. Further work should focus on the exploration of high quality light harvesting materials with suitable band gap and proper HTM, combining with the optimization of the device structure. 


\section{Recombination control}

In QDSCs, apart from the preferred charge transfer processes, there are also several recombination paths competing with these favorable processes. It is well known that non-radiative charge recombination can seriously deteriorate the performance of the device. ${ }^{33,38}$ For QDSCs charge recombination is particularly serious compared to DSCs due to the existence of defect states in QD sensitizers, the low QDs loading amount induced large portion of bare $\mathrm{TiO}_{2}$ surface directly exposed to the electrolyte, and the complex chemical activity of the commonly used polysulfide electrolyte. Therefore, the suppression of charge recombination in QDSCs is a big issue that should be addressed to obtain desirable photovoltaic performance.

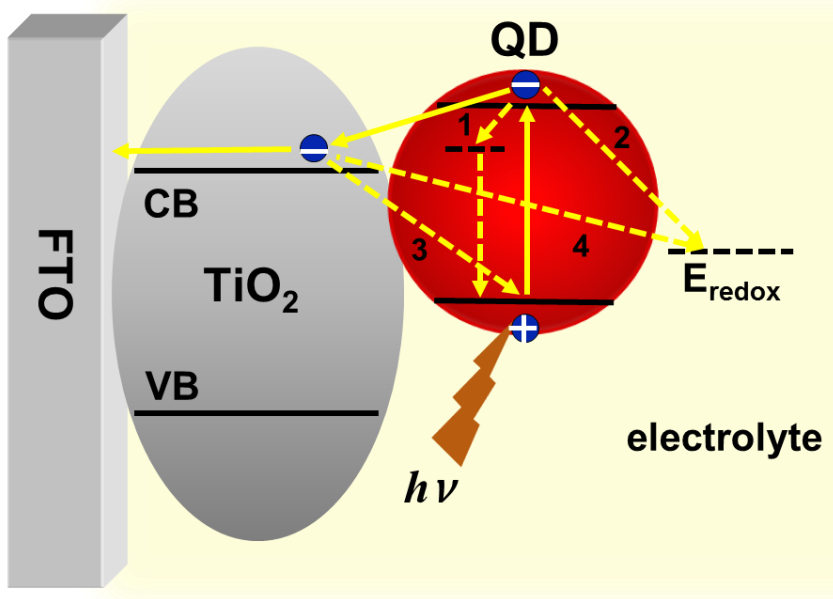

Fig. 30 Schematic illustration of the main charge recombination routes in QDSCs.

As shown in Fig. 30 with dashed arrows, there are four main recombination pathways in the QDSCs: (1) charge recombination inside QD through defect states, (2) recombination of excited electron in QD with oxidized species in electrolyte at QD/electrolyte interface, (3) back transfer of electrons in the $\mathrm{TiO}_{2}$ to $\mathrm{QD}$ at $\mathrm{TiO}_{2} / \mathrm{QD}$ interface, and (4) recombination of electrons in $\mathrm{TiO}_{2}$ with electrolyte at $\mathrm{TiO}_{2} /$ electrolyte interface. ${ }^{38}$ It can be seen that the defect states in QD and the large area of bare $\mathrm{TiO}_{2}$ surface aggravate the charge recombination in QDSCs compared to DSCs, where $\mathrm{TiO}_{2}$ is practically fully covered by the dye, or polymer solar cells. In recent years, considerable efforts have been made to address the recombination issue. In general, the reported means for recombination controlling in QDSCs can be summarized into two kinds: materials engineering and interface engineering, which will be discussed in detail in the following section. 


\subsection{Materials engineering}

Since one of the major charge recombination processes takes place inside QD through defect states, high quality QD sensitizer becomes a prerequisite for high efficiency solar cells. In the early investigation stage of QDSCs, QDs were usually grown directly on $\mathrm{TiO}_{2}$ substrate through SILAR or CBD methods. ${ }^{36,101}$ As mentioned in the above section, QDs formed by this in situ approach usually possess high density of defect states due to the poor crystallinity and large number of dangling bonds at the surface, leading to severe charge recombination inside QDs or back electron transfer from $\mathrm{TiO}_{2}$ to QDs. Therefore, the adoption of high quality pre-synthesized QD sensitizer is an effective route to improve the photovoltaic performance of QDSCs. ${ }^{36,457}$ On one hand, the pre-synthesized colloidal QDs possess lower density of inner and surface defect states benefitting from the well-developed organometallic synthesis method. On the other hand, the pre-prepared QD provides us more opportunities to realize a better control over the composition and structure of the QDs. Thus, it's not surprising that the adoption of pre-synthesized QD as sensitizer prominently promoted the development of QDSCs in the past five years. ${ }^{74,85,109,111,115-118,219,425}$ Accordingly, much effort has been made to design the composition and structure of the QDs in the view of charge recombination control. Among these, the construction of core/shell structure and the alloying strategy have been demonstrated to be efficient to reduce charge recombination loss.

The core/shell structured QDs are an attractive material to operate the recombination design since their photoelectric properties can be facilely tailored by the shell materials. ${ }^{270,271}$ Among them, type-II structured QD owns potential to reduce the charge recombination inside QD. For type-II structured QDs, both the CB and VB of the core material located either lower or higher than the shell material, resulting in the spatial separation of charge carriers. Accordingly, if the $\mathrm{CB}$ and VB of the core material are both higher than the shell material, the electron is mostly confined to the shell layer and the hole is mostly confined to the core layer. $268,270,271$ It is noted that this spatial separation of electron and hole reduces the possibility of charge recombination within QDs. Inspired by these unique properties, several attempts have been made to apply type-II QDs as sensitizers in QDSCs, including $\mathrm{ZnSe} / \mathrm{CdS}$, $\mathrm{ZnTe} / \mathrm{ZnSe}, \mathrm{CdTe} / \mathrm{CdSe}, \mathrm{ZnTe} / \mathrm{CdSe}$ etc., and a record PCE of $7.17 \%$ was achieved for $\mathrm{ZnTe} / \mathrm{CdSe}$ based QDSCs. ${ }^{74,85,217,416}$ The details of the related works have been discussed in the above section. It is noted that the obtained $V_{\text {oc }}$ of QDSCs based on these type-II QDs are especially higher compared to other QDs based one, partially due to the inhibited charge recombination inside QDs. 

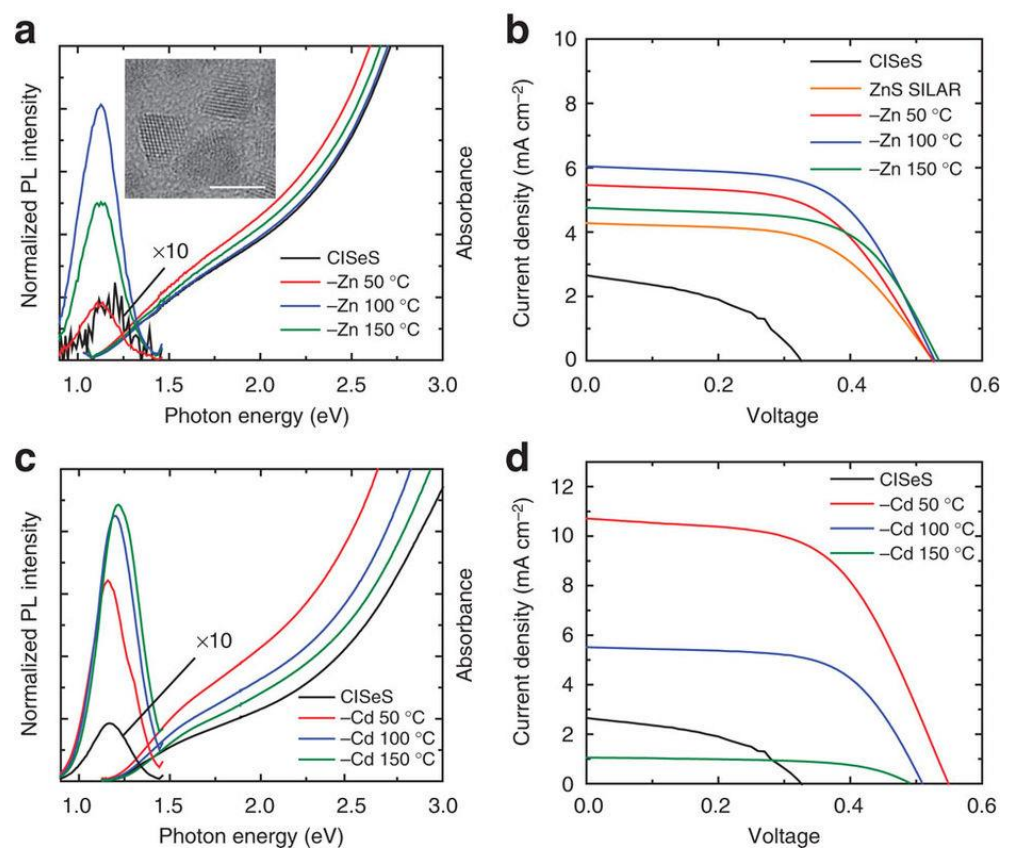

Fig. 31 (a) Absorption and photoluminescence (PL) spectra of the QDs before and after exposure to $\mathrm{Zn}$-oleate at various temperatures after recapping with tert-butylamine (tBA). Inset: TEM image of $4.5 \mathrm{~nm} \mathrm{CuInSe}{ }_{1.4} \mathrm{~S}_{0.6}$ QDs. Scale bar, $5 \mathrm{~nm}$. (b) $J-V$ curves for QDs with varying degrees of Zn-cation exchange, or with ZnS SILAR post-treatment of the QD-infused anode (orange line). (c) Absorption and PL spectra of the QDs before and after exposure to Cd-oleate and recapping with tBA. (d) $J-V$ curves for QDs with varying degrees of Cd-cation exchange controlled by reaction temperature. Reprinted with permission from ref. 78 . Copyright (2013) Macmillan Publishers Limited.

Besides, type-I structured core/shell QDs also show their superiority in controlling charge recombination process. In type-I structured QDs, the shell materials have a higher CB edge and lower VB edge than those of core materials. ${ }^{270}$ On one hand, the wide band gap shell acts as a barrier layer, which can prevent the photon-generated electron leakage from QD to electrolyte. On the other hand, the epitaxial growth of wide band gap shell material significantly reduces the trap state density of the core QD, so the processes of internal charge recombination inside QD and electron back transfer from $\mathrm{TiO}_{2}$ to the trap states of QDs are retarded consequently. ${ }^{38}$ However, it should be noted that the shell can also act as a barrier in preventing the electron injection from QD to electron acceptor. ${ }^{116}$ Therefore, the thickness of the shell material should be thin enough (generally less than 1-2 $\mathrm{nm}$ ) to guarantee efficient electron extraction. The cation exchange method is a convenient way to fabricate a thin shell layer around the core QDs to form the quasi type-I structure. For example, Zhong and 
coworkers applied cation exchange approach to prepare type-I CuInS $\mathrm{I}_{2} / \mathrm{ZnS}$ core/shell QDs. ${ }^{116}$ The photoluminescence (PL) quantum yield of the as prepared QD was significantly improved after the formation of the $\mathrm{ZnS}$ shell layer through cation exchange process. Accordingly, the PCE of the cell was improved from $5.05 \%$ ( $\mathrm{CuInS}_{2}$ based) to $7.04 \%$ $\left(\mathrm{CuInS}_{2} / \mathrm{ZnS}\right.$ based) benefitting from the inhibited charge recombination and prolonged electron lifetime. Klimov and coworkers compared the different effects of cation exchange in $\mathrm{CuInSe}_{1-\mathrm{x}} \mathrm{S}_{\mathrm{x}}$ QDs with use of $\mathrm{Zn}^{2+}$ and $\mathrm{Cd}^{2+}$ ions as the exchange cation. ${ }^{78}$ It was found that both the $\mathrm{Zn}^{2+}$ and $\mathrm{Cd}^{2+}$ cation exchange can contribute to an improvement in the PL quantum yield as well as the resultant solar cell performance, while the enhancement is greater for the $\mathrm{Cd}^{2+}$ based one, and a certified PCE of 5.1\% was obtained (Fig. 31).

Recently, alloying strategy has been demonstrated to be a convenient way to improve the photoelectronic properties of QDs as well as the photovoltaic performance of the related solar cells. ${ }^{115,223,274}$ As discussed above, there are two conflicting effects of the shell layer in type-I structured core/shell QDs, reducing surface trapping defects but retarding electron injection and hole scavenge simultaneously. What's more, the formation of the thin shell layer can only reduce the surface defect states of QDs, while it is powerless to tailor the defect states inside QDs. In contrast, alloying a wide band gap material into the native QD can resolve these problems simultaneously. It has been shown that the alloying strategy could not only reduce the density of trap defects both at the surface and inside QD due to the hardened lattice structure and decreased atomic intradiffusion, but also favor the electron injection due to the upshift of $\mathrm{CB}$ edge. Zhong and coworkers alloyed $\mathrm{ZnSe}$ and $\mathrm{Ga}_{2} \mathrm{Se}_{3}$ in the native CISe QDs to form $\mathrm{Zn}-\mathrm{Cu}-\mathrm{In}-\mathrm{Se}$ or $\mathrm{Ga}-\mathrm{Cu}-\mathrm{In}-\mathrm{Se} \mathrm{QDs}$ through "simultaneous nucleation and growth" approach. ${ }^{15,223}$ The PCE of the QDSCs can be improved by more than $20 \%$ with use of the alloying strategy in QDs sensitizers and a record PCE of $11.66 \%$ was obtained. The enhancement in the solar cell performance can be mainly attributed to the lower density of trap states in QDs that inhibiting charge recombination process and the upshift of CB edge that improving the electron injection efficiency. 


\subsection{Interface engineering}

In charge recombination control, besides the amelioration of the quality of QD sensitizers to suppress the QD-related recombination processes for, other attention was mainly paid on the recombination processes taking place at the $\mathrm{TiO}_{2} / \mathrm{QD} /$ electrolyte interface. ${ }^{32,33,38}$ In the photoanode, QDs and the bare $\mathrm{TiO}_{2}$ surface contacted directly with electrolyte. This will facilitate the electron being captured by the oxidized species in the electrolyte. Thus, the modification of the $\mathrm{TiO}_{2} / \mathrm{QD} /$ electrolyte interface is crucial to realize high photovoltaic performance of QDSCs. As a whole, the interface modification techniques can be classed into three kinds: coating strategy with use of inorganic wide band gap materials, organic molecule treatment, and the electrolyte modification.
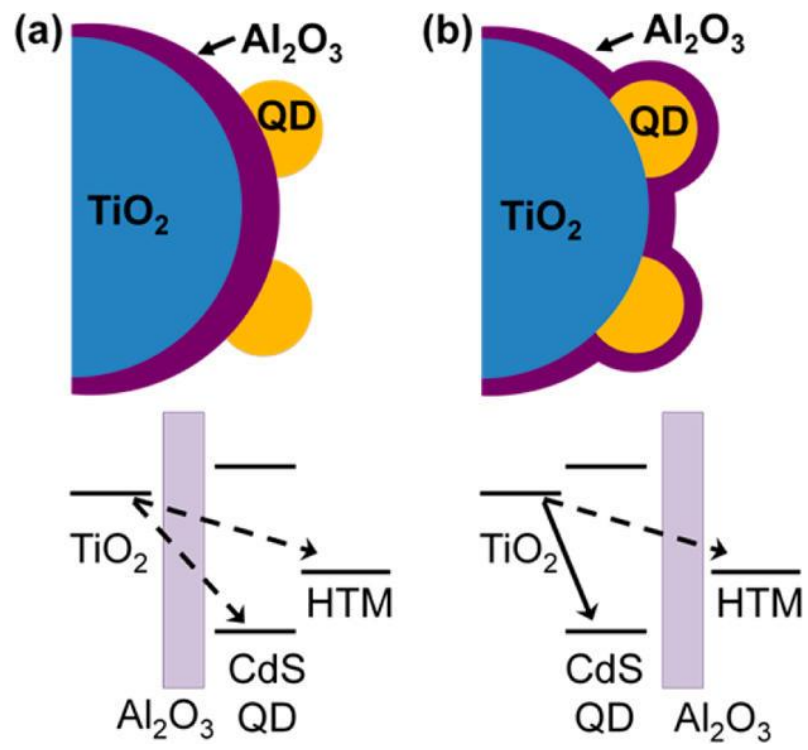

Fig. 32 Schematic of barrier layer configurations (not to scale) available in quantum dot-sensitized solar cells: (a) $\mathrm{TiO}_{2} / \mathrm{Al}_{2} \mathrm{O}_{3} / \mathrm{QD}$ and (b) $\mathrm{TiO}_{2} / \mathrm{QD} / \mathrm{Al}_{2} \mathrm{O}_{3}$, resulting, respectively, from deposition of the $\mathrm{Al}_{2} \mathrm{O}_{3}$ layer before and after the CdS QDs. Spiro-OMeTAD is employed as the hole-transport material (HTM). Arrows indicate undesirable recombination pathways; pathways that may be blocked by the $\mathrm{Al}_{2} \mathrm{O}_{3}$ barrier layer are shown by dashed arrows. Reprinted with permission from ref. 458. Copyright (2013) American Chemical Society.

5.2.1. Coating strategy. The coating strategy on the photoanode with use of wide band gap materials to prevent the direct contacting between photoanode and electrolyte is believed to be an efficient way to reduce electron recombination losses at the photoanode/electrolyte interface. Among these, $\mathrm{ZnS}$ coating layer is the most popular and common used one in 
QDSCs and showed dramatically positive effect in improving solar cell performance. ${ }^{117,459-464}$ The concept of $\mathrm{ZnS}$ coating treatment on semiconductor to tailor the interface characteristics was first carried out by Huang and coworkers, in which they coated $\mathrm{TiO}_{2} / \mathrm{PbS} / \mathrm{CdS}$ electrode by a $\mathrm{ZnS}$ layer through SILAR method to protect the $\mathrm{PbS}$ and $\mathrm{CdS}$ particles from photocorrosion. ${ }^{460}$ It was found that the photovoltaic performance of the solar cells was improved significantly after the $\mathrm{ZnS}$ treatment, especially in the $V_{\mathrm{oc}}$ value. This work indicated that the $\mathrm{ZnS}$ coating layer can not only protect the QDs from photocorrosion, but also showed a suppressing effect on the charge recombination process. After that, Toyoda and Shen and coworkers introduced this treatment to QDSCs and systematically investigated the effect of $\mathrm{ZnS}$ coating layer on the solar cell performance. ${ }^{99,459,464}$ It has been demonstrated that the $\mathrm{ZnS}$ treatment acts two roles in the performance of QDSCs: one is to passivate QDs surface and the other is to block the bare $\mathrm{TiO}_{2}$ surface. As a result, an improvement of $74 \%$ in the final PCE can be achieved. Since then, the $\mathrm{ZnS}$ coating treatment has been adopted as a typical process in the fabrication of QDSCs. Meanwhile, ZnSe was also used as a passivation layer in QDSCs. ${ }^{465-469}$ For example, Cao and coworkers compared the effect of $\mathrm{ZnS}$ and $\mathrm{ZnSe}$ passivation layer on the performance of QDSCs. ${ }^{466}$ They found that the ZnSe passivation layer was superior to $\mathrm{ZnS}$ passivation layer in improving the PCE of QDSCs. The ZnSe passivation layer can not only improve the light harvesting capability of the photoanode, but also showed a better charge recombination inhibition effect. Finally, a PCE of $6.4 \%$ was achieved for CdS/CdSe QDSCs with a ZnSe passivation layer, about 30\% higher than the $\mathrm{ZnS}$ passivation layer based one (4.9\%).

Apart from $\mathrm{ZnS}$ or $\mathrm{ZnSe}$ passivation layer, metal oxides with wide band gap are also investigated as coating layer to inhibit charge recombination at photoanode/electrolyte interface in QDSCs. ${ }^{113,114,117,256,458,470-473}$ This is motivated by the corresponding works in DSCs, in which the insulating metal oxide treatment on photoanode has been widely investigated for the purpose of recombination control. ${ }^{474-477}$ It has been revealed that the main function of the metal oxide layer coating is to block electron back transfer process, thus improving the $V_{\mathrm{oc}}$ of the cell device. Zaban and coworkers deposited a thin amorphous $\mathrm{TiO}_{2}$ layer on the QDs sensitized film. ${ }^{471}$ The results showed that both the PCE and stability of the CdS based QDSCs were remarkably improved in $\mathrm{I}^{-} / \mathrm{I}_{3}{ }^{-}$electrolyte. Besides, Zaban and coworkers also used $\mathrm{MgO}$ coating to modify the interfaces. ${ }^{472}$ They deposited conformal $\mathrm{MgO}$ by EPD method on $\mathrm{TiO}_{2}$ electrode before or after QDs deposition, illustrating that the interface between $\mathrm{TiO}_{2}$ and QDs is also crucial for the recombination process as the other 
interfaces in the $\mathrm{TiO}_{2} / \mathrm{QDs} /$ electrolyte triple junction. Bent and coworkers deposited $\mathrm{Al}_{2} \mathrm{O}_{3}$ barrier layers either before or after the QD deposition on $\mathrm{TiO}_{2}$ film by ALD method to fabricate $\mathrm{CdS}$ or PbS based solid-state QDSCs (Fig. 32). ${ }^{458}$ It was found that the $\mathrm{Al}_{2} \mathrm{O}_{3}$ barrier layer can act as a tunneling barrier to suppress charge recombination and increase electron lifetimes in $\mathrm{TiO}_{2}$. With the optimization of $\mathrm{Al}_{2} \mathrm{O}_{3}$ layer thickness, the obtained $V_{\text {oc }}$ and $\mathrm{PCE}$ were increased obviously.
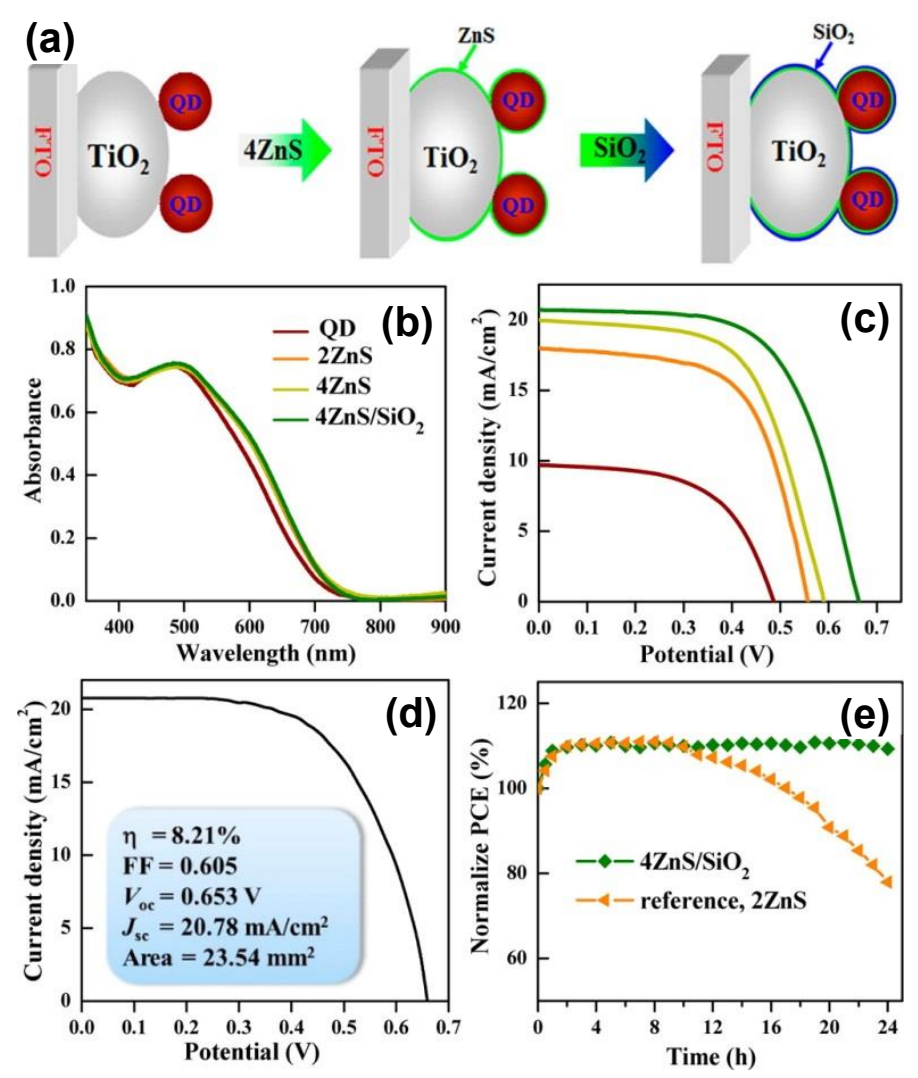

Fig. 33 (a) Schematic showing the sequential overcoating of $\mathrm{ZnS}$ and $\mathrm{SiO}_{2}$ thin layer around the QD-sensitized $\mathrm{TiO}_{2}$ film electrode. (b) Absorption spectra of photoanode films after different coatings. (c) $J-V$ curve of samples prepared with different coatings after QD sensitization. (d) Certified efficiency of QDSC prepared with $4 \mathrm{ZnS} / \mathrm{SiO}_{2}$ coating. (e) Cell efficiency normalized to the initial efficiency for samples with different coating measured during $24 \mathrm{~h}$ period under continuous 1 sun illumination. Reprinted with permission from ref. 117. Copyright (2015) American Chemical Society.

Zhong and coworkers reported a robust coating treatment to tailor the interface charge recombination processes, in which they applied a novel double coating layer by sequentially depositing $\mathrm{ZnS}$ and $\mathrm{SiO}_{2}$ on the QD sensitized $\mathrm{TiO}_{2}$ electrode (Fig. 33). ${ }^{117}$ The density 
functional theory (DFT) calculations unraveled that $\mathrm{ZnS}$ or $\mathrm{SiO}_{2}$ single coating layer can reduced the surface electron density of states (DOS) by 2 orders of magnitude, while the double coating layer can further reduce the DOS by 3 orders of magnitude. As a result, the PCE of the constructed cell was boosted from $7.17 \%$ (single $\mathrm{ZnS}$ layer) to $8.55 \%\left(\mathrm{ZnS} / \mathrm{SiO}_{2}\right.$ double layer). Besides, they introduced an additional amorphous metal oxyhydroxide as a buffer layer between the QDs sensitized film and $\mathrm{ZnS} / \mathrm{SiO}_{2}$ blocking layer to further suppress charge recombination at the interface. ${ }^{113,114} \mathrm{~A}$ series of metal oxyhydroxide have been overcoated on CdSeTe QD sensitized photoanodes via a hydrolysis and condensation process from the corresponding metal chloride aqueous solution to investigate their effect on the photovoltaic performance. It was found that the introduction of am- $\mathrm{TiO}_{2}, \mathrm{am}-\mathrm{ZrO}$, and am- $\mathrm{Nb}_{2} \mathrm{O}_{5}$ as buffer layer showed beneficial effects on the improvement of the cell performance due to the further suppressed charge recombination processes, and the PCE was further boosted to $9.73 \%$ with $\mathrm{ZrOCl}_{2}$ treatment on photoanode.

Unlike the above mentioned wide band gap semiconductor or insulating inorganic materials overcoating strategy aiming at isolating photoanode with electrolyte, and reducing photogenerated electron leakage at the photoanode/electrolyte interface, p-type semiconductor $\mathrm{CuS}$ overlayer has been used to facilitate hole transfer to the electrolyte from QD sensitizers. ${ }^{192,462,478}$ In an example reported by Ghosh et $a l,{ }^{479} \mathrm{CdS}$ layer was first overcoated around QD sensitized photoanode, and then $\mathrm{Cd}^{2+}$ was partially replaced by $\mathrm{Cu}^{2+}$ to form CuS layer. With the formation of CuS layer, the PCE of the champion cell was $4.03 \%$, which is about $12 \%$ higher than the one with normal $\mathrm{ZnS}$ coating. EIS measurements disclosed that the CuS layer acted as a hole transporting buffer layer, increasing the charge collection efficiency. Therefore, this strategies will help to overcome the inherent disadvantage of slower hole transfer rate in standard QDSCs, which is one of the key factors limiting the performance of QDSCs.

5.2.2. Organic molecular treatment. Apart from the inorganic coating layer treatment, photoanode interface modification with use of organic molecules was also demonstrated to be effective for the suppression of charge recombination. ${ }^{480-485}$ For example, Mora-Seró and coworkers showed that molecular dipoles (DT) assisted $\mathrm{ZnS}$ treatment can give a better control of the recombination dynamics as well as the charge injection process. ${ }^{480}$ It was found that the sequence of DT and $\mathrm{ZnS}$ treatment can significantly influence the final performance of solar cells, and the optimized sequence of the treatment $(\mathrm{DT}+\mathrm{ZnS})$ resulted in a dramatic $600 \%$ increase of PCE compared to the reference cell without treatment. Furthermore, they 
also investigated the passivation effect of different organic molecules, including ethanedithiol (EDT), ethylenediamine (ETDA), thioglycolic acid (TGA), dimethylamine (DMA), and formic acid (FA). ${ }^{481}$ It was found that the treatment with use of molecules containing amine or thiol groups can enhance the PCE of QDSCs, while the treatment by molecules with acid groups would lead to a negative effect. It should be noted that investigation of organic molecule treatment on QD sensitized photoanode was obtained via the in situ growth of QD sensitizers on $\mathrm{TiO}_{2}$ film electrode. In this condition, no capping ligand was presented on the surface of QD sensitizers, so that the organic molecules can easily absorb on the QDs. It is not certain whether this organic molecule treatment can provide a similar positive effect in ligand-capped pre-prepared QD sensitizer based QDSCs. In the case of ex situ prepared QD sensitizers, thiol coadsorbents (such as thioglycolic acid (TGA), 3-mercaptopropionic acid (MPA), glutathione (GSH), and cysteine) were added into the QD solution prepared in aqueous media during the QD deposition process. ${ }^{248}$ Experimental results indicated that the photovoltaic performance of the resulting QDSCs were dependent on the type and concentration of the thiol coadsorbent used. With the use of TGA coadsorbents, the PCE of the $\mathrm{CuInS}_{2}$ QDSCs (5.90\%) was 20 times higher than that of the control cell without TGA coadsorbents $(0.29 \%)$. The versatility of this strategy was demonstrated in the fabrication of QDSCs with use of $\mathrm{AgInS}_{2}$ or CdSeTe QDs prepared in aqueous media. The improved performance was ascribed to reducing the disulfides, and varying the conduction band edge of $\mathrm{TiO}_{2}$. However the effect of coadsorbents in increasing QD loading amount is prominent, which can explain the enhanced $J_{\text {sc }}$ and $V_{\text {oc }}$.

5.2.3. Electrolyte modification. As discussed above, the complex nature of polysulfide electrolyte brings forward severe charge recombination. Previous work has demonstrated that the rate of charge recombination from $\mathrm{TiO}_{2}$ to polysulfide electrolyte is at least 2 orders of magnitude greater than that in $\mathrm{I}^{-} / \mathrm{I}_{3}{ }^{-}$redox couple based system. ${ }^{32}$ This should be seriously taken into account to design high efficiency QDSCs. An alternative way to suppress the charge recombination is the polysulfide electrolyte modification with use of additives to tune its electrochemical properties.

Zhong et al. found that the addition of water-soluble polymers, such as PEG and PVP in polysulfide electrolyte can distinctly inhibit the charge recombination rate at the $\mathrm{TiO}_{2} / \mathrm{QD} /$ electrolyte interface. ${ }^{205,424}$ They proposed that the polymer additives in the polysulfide electrolyte can serve as a protective layer over both $\mathrm{TiO}_{2}$ and QDs surfaces by the steric hindrance effect, thus inhibiting the unwanted charge recombination from $\mathrm{TiO}_{2}$ to 
polysulfide electrolyte and improving the resultant photovoltaic performance. Meng et al. reported that the addition of fumed $\mathrm{SiO}_{2}$ nanoparticles in polysulfide electrolyte also resulted in an inhibition effect of the charge recombination. ${ }^{425}$ It was demonstrated that the $\mathrm{SiO}_{2}$ nanoparticles in the electrolyte can create an energy barrier at QDs/electrolyte as well as $\mathrm{TiO}_{2} /$ electrolyte interfaces, consequently retarding the charge recombination processes. However, it should be noted that the intrinsic mechanism of charge recombination inhibition with use of these additives in polysulfide electrolyte has not been understood very clearly now. 


\section{Stability issue}

For all kinds of emerging solar cells, long-term stability is requisite to realize their commercial application. Up to now, long-term stability is one of the bottlenecks that limit the practical application for almost all kinds of emerging solar cells. ${ }^{486-491}$ In QDSCs, however, few studies are focused on the stability investigation, and the cell stability is still far from satisfactory level. Therefore, for the further development of this kind of solar cells, much effort should be devoted to address the stability issue. The stability of QDSCs is mainly determined by the following factors: (1) the chemical stability of QDs in electrolyte; (2) the chemical and mechanical stability of counter electrode used in QDSCs; (3) the stability of redox couple in electrolyte; and (4) sealing technology to avoid the leakage and volatilization of electrolyte (for the liquid-junction device). The summary of stability tests for QDSCs is shown in Table 6.

$\mathrm{I}^{-} / \mathrm{I}_{3}{ }^{-}$redox couple is the most popular choice in DSCs, while most QDs are unstable in this system. Although polysulfide electrolyte $\left(\mathrm{S}^{2-} / \mathrm{S}_{\mathrm{n}}{ }^{2-}\right)$ was found to be suitable to stabilize QDs and therefore offer a relatively stable cell device, photo-corrosion of QDs in polysulfide electrolyte can still take place due to the complex chemical properties of polysulfide redox couple, resulting in the deterioration of the cell stability. ${ }^{75,253}$ In addition, the chemical and mechanical stability of CEs can also affect the stability of the cell device. For example, brass foil based $\mathrm{Cu}_{2} \mathrm{~S}$ is commonly used as $\mathrm{CE}$ in QDSCs, while this CE suffers from continuous corrosion by the polysulfide electrolyte, resulting in the leakage of the electrolyte eventually. ${ }^{397}$ Therefore, to improve the stability of QDSCs, the exploiting of highly stable QDs, robust CE and cell sealing technique should be taken into account simultaneously.

The good chemical stability of QDs towards light, heat, and electrolyte is prerequisite to realize good device stability. Various QDs, such as $\mathrm{PbS}, \mathrm{CdTe}$, and $\mathrm{Sb}_{2} \mathrm{~S}_{3}$ have been proven to be unstable in traditional polysulfide electrolyte. ${ }^{97,253,448}$ Zhong et al. demonstrated an improvement in the solar cell stability with use of an alloyed $\mathrm{CdSe}_{\mathrm{x}} \mathrm{Te}_{1-\mathrm{x}} \mathrm{QD}$ with good stability in polysulfide electrolyte. ${ }^{111}$ The fabricated QDSCs exhibited good stability for more than $500 \mathrm{~h}$ (Fig. 34). It is noted that many subsequent studies on the stability improvement were based on this kind of state-of-art sensitizer. ${ }^{205,425,426,432,473}$ In addition, the doping strategy in QD sensitizers was also found to favor the stability of QDSCs. ${ }^{107,305,492}$ For example, Kamat et al. reported that the QDSCs with use of Mn doped CdS/CdSe QDs as sensitizer showed good stability under continuous illumination of $100 \mathrm{~mW} \mathrm{~cm}{ }^{-2}$ for $2 \mathrm{~h}^{107}$ 
Gopi et al. also demonstrated that the cells based on Mn doped CdS QDs displayed better stability compared to the undoped one. ${ }^{305}$
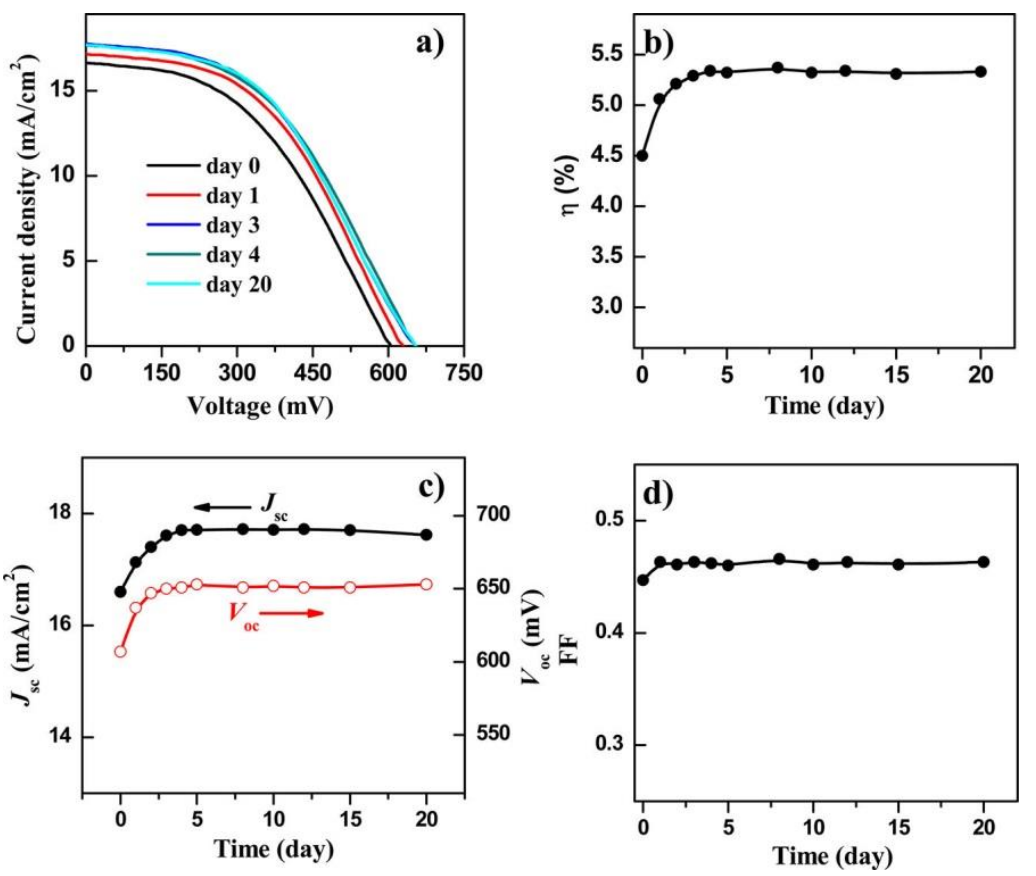

Fig. 34 Temporal evolution of $J-V$ curves (a) and photovoltaic parameter values, $\eta$ (b), $J_{\text {sc }}$, $V_{\mathrm{oc}}$ (c), and $\mathrm{FF}(\mathrm{d})$, for the $\mathrm{CdSeTe} 800$ cells based on $\mathrm{Cu}_{2} \mathrm{~S}$ counter electrodes via electrodeposition of $\mathrm{Cu}$ on FTO glass. Reprinted with permission from ref. 111. Copyright (2013) American Chemical Society.

Another way to improve the stability of QDSCs is photoanode post-treatment with overcoating an isolating wide band gap semiconductor. ${ }^{117,460,471,473,493,494}$ On one side, the coating layer can prevent QDs from directly contacting with electrolyte, thus reducing the photo-corrosion of QDs in electrolyte. On the other side, the coating layer around photoanode can protect QDs from the attack of oxygen molecule so that improving the chemical stability of QDs. For example, the commonly used $\mathrm{ZnS}$ or $\mathrm{ZnSe}$ passivation layer was found to be capable of improving the stability of QDSCs. ${ }^{460,493,494}$ In addition, metal oxide coating layer on photoanode can also favor the stability of the device. ${ }^{471}$ For instance, Zaban et al. presented a coating strategy on $\mathrm{CdS}$ sensitized $\mathrm{TiO}_{2}$ film with use of an amorphous $\mathrm{TiO}_{2}$ layer to improve the cell stability in $\mathrm{I}^{-} / \mathrm{I}_{3}{ }^{-}$electrolyte. ${ }^{471}$ The amorphous $\mathrm{TiO}_{2}$ coating protected the QDs from the severe corrosion of $\mathrm{I}^{-} / \mathrm{I}_{3}{ }^{-}$redox couple and therefore obtained a better performance compared to the uncoated one. Zhong et al. found that a $\mathrm{ZnS} / \mathrm{SiO}_{2}$ double layer coating on photoanode was beneficial for the stability improvement of QDSCs. ${ }^{117}$ The constructed cell with this double layer coating exhibited no degradation in a course of $24 \mathrm{~h}$ 
under 1 sun continuous irradiation, significantly better than the single $\mathrm{ZnS}$ coated one (Fig. 29d). They also demonstrated that a $\mathrm{ZnS}$ and metal hydroxide composite passivation layer favored the stability of QDSCs benefitting from the better protection effect of the passivation layer to QDs. ${ }^{473}$
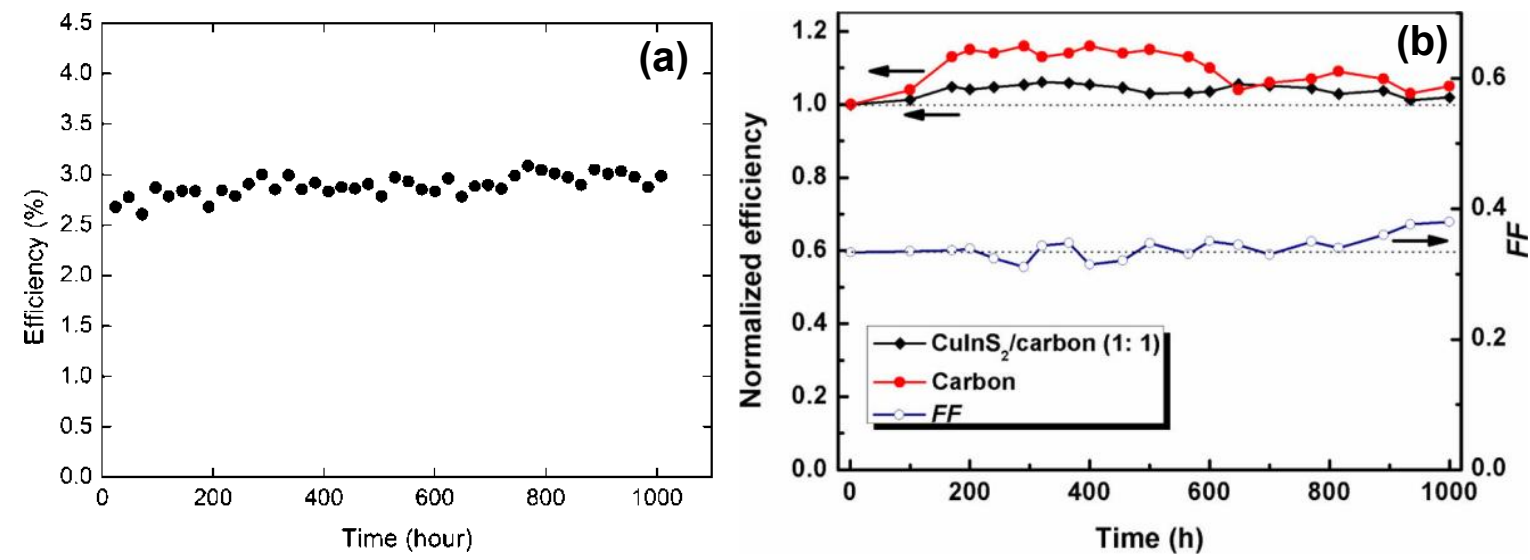

Fig. 35 (a) Efficiency test of PbS/CB composite CE based sealed QDSCs over $1000 \mathrm{~h}$. The cells are kept in room conditions and measured under AM $1.5100 \mathrm{~mW} \mathrm{~cm}{ }^{-2}$ illumination every day. Reprinted with permission from ref. 398. Copyright (2012) American Chemical Society. (b) Normalized efficiency the CdS/CdSe QDSCs fabricated with $\mathrm{CuInS}_{2} /$ carbon composite (weight ratio 1:1) and carbon electrodes versus conservation time. Reprinted with permission from ref. 399. Copyright (2013) American Chemical Society.

Another factor that limits the stability of QDSCs is the nature of the CE catalytic materials used. To overcome the stability issue induced by brass foil based CEs, several groups developed novel CEs on FTO substrate to improve the stability of the cell device. ${ }^{342,349,398,399,411,495}$ For example, Meng's group prepared two kinds of composite CEs, $\mathrm{PbS} /$ carbon black and $\mathrm{CuInS}_{2} /$ carbon black on FTO substrate. ${ }^{398,399}$ It was proposed that the framework of carbon black used in the composite CE together with PVDF binder provided good physical contact between nanoparticles and the FTO substrate and therefore can improve the long-term stability of the CE. The composite CE based cells showed excellent stability over $1000 \mathrm{~h}$ without degradation under room light conditions (Fig. 35). It is noted that this is the longest period of the stability test for QDSCs. Wang et al. prepared hierarchical CuS/FTO CEs through a facile electrochemical deposition method and exhibited excellent chemical and electrochemical stability in polysulfide electrolyte. ${ }^{342}$ The corresponding QDSC device presented high illumination and conservation stability for 2 and $240 \mathrm{~h}$, respectively. Zhong et al. deposited a copper film on FTO substrate through 
electrodeposition method and then $\mathrm{Cu}_{2} \mathrm{~S} / \mathrm{FTO} \mathrm{CE}$ was formed by dipping $\mathrm{Cu} / \mathrm{FTO}$ into polysulfide solution. ${ }^{349}$ The cell based on this $\mathrm{Cu}_{2} \mathrm{~S} / \mathrm{FTO} \mathrm{CE}$ showed good stability over $10 \mathrm{~h}$ without degradation under continuous irradiation at $100 \mathrm{~mW} \mathrm{~cm}{ }^{-2}$ (Fig. 36). Gopi et al. prepared a $\mathrm{NiS} / \mathrm{PbS}$ composite $\mathrm{CE}$ on FTO using $\mathrm{CBD}$ technique and the PCE of the fabricated QDSC showed no degradation over $10 \mathrm{~h}$ under room light conditions. ${ }^{411}$
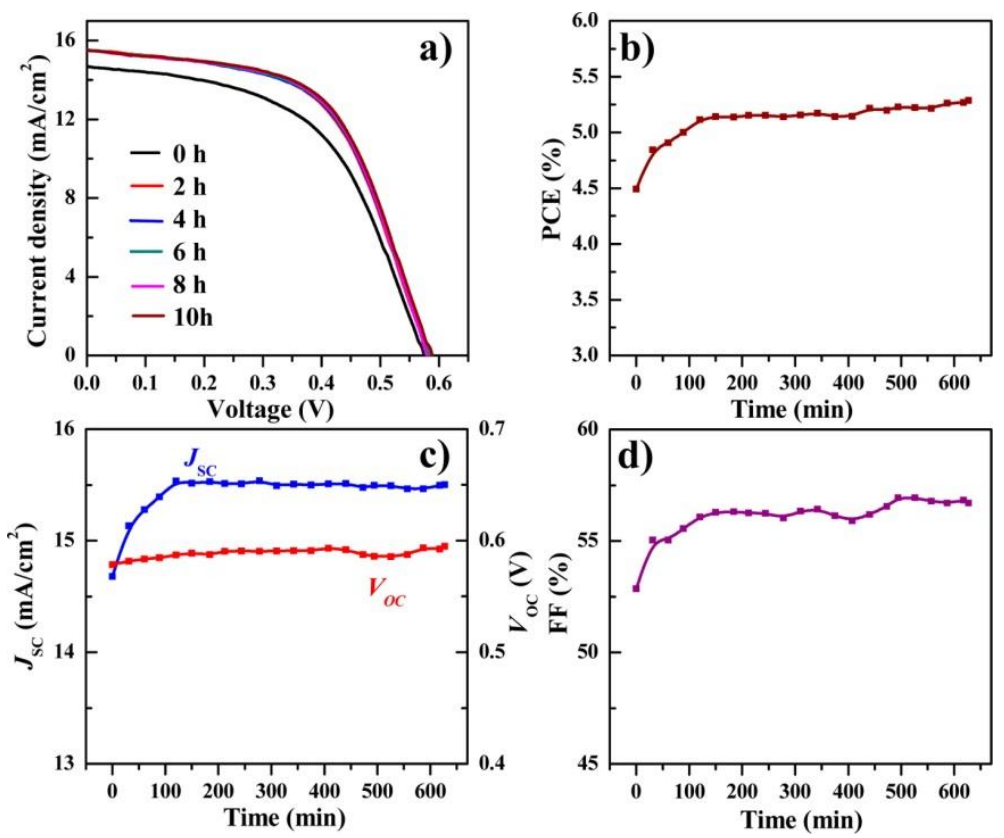

Fig. 36 Temporal evolution of $J-V$ curves (a), PCE (b), $J_{\mathrm{sc}}, V_{\mathrm{oc}}(\mathrm{c})$, and FF (d) for the CdSe QDSCs based on $\mathrm{Cu}_{2} \mathrm{~S} / \mathrm{FTO} \mathrm{CE}$. The $J-V$ curves were tested under irradiation by an $1.5 \mathrm{G}$ solar simulator at intensity of $100 \mathrm{~mW} \mathrm{~cm}^{-2}$. Reprinted with permission from ref. 349 . Copyright (2014) American Chemical Society.

In addition to the above mentioned methods to address the stability issue, another efficient way to improve the stability of QDSCs is the modification of electrolyte. ${ }^{205,391,424-426,431,432,496}$ As discussed above, the relative high chemical activity of polysulfide electrolyte leads to the corrosion of QDs and therefore damage the stability of the resultant device. Hence, the modification of electrolyte with use of additives to tune its chemical activity or the physical features is a potential way to improve the stability of QDSCs. Zhong's group adopted water-soluble polymers, such as poly(ethylene glycol) (PEG) and poly(vinyl pyrrolidone) (PVP) as additives in polysulfide electrolyte. ${ }^{205,424}$ It was found that both additives were favorable for the improvement of cell stability. Meng et al. reported a fumed $\mathrm{SiO}_{2}$ nanoparticles modified polysulfide electrolyte to improve the photovoltaic performance of QDSCs. ${ }^{425}$ An improvement in the solar cell stability was achieved employing $\mathrm{SiO}_{2}$ 
NP-modified electrolyte, in which only a $6.3 \%$ drop in the PCE was found for the cell based on this modified electrolyte after 42 days of storage, whereas the PCE of the reference cell dropped by $23.3 \%$ during this period. Similar results were also obtained by Zhong et al., in which tetraethyl orthosilicate (TEOS) was used as additive in polysulfide electrolyte to form two-dimensional amorphous $\mathrm{SiO}_{2}$ film, showing a distinct improvement in the cell stability. ${ }^{391}$ Besides, it is well known that liquid electrolyte suffers from the limitation of volatilization and leakage, seriously hampering the stability of the cell device. Therefore, the fabrication of solid-state or quasi-solid-state solar cells is another way to obtain highly stable QDSCs. For example, Meng et al. applied natural polysaccharide konjac glucomannan (KGM) as the polymer matrix to prepare gel electrolyte and significantly improved the cell stability. ${ }^{428}$ Kim et al. also found an improvement in stability with use of poly(ethylene glycol) dimethyl-ether (PEGDME) supported gel polysulfide electrolyte. ${ }^{497}$ Zhong et al. used sodium polyacrylate (PAAS) and sodium carboxymethylcellulose (CMC-Na) as gelators to prepare quasi-solid-state gel electrolytes for QDSCs, and both of the gel electrolytes contributed to an improvement of the cell stability under continuous irradiation for $90 \mathrm{~h}$ (Fig. 37). ${ }^{426,432}$
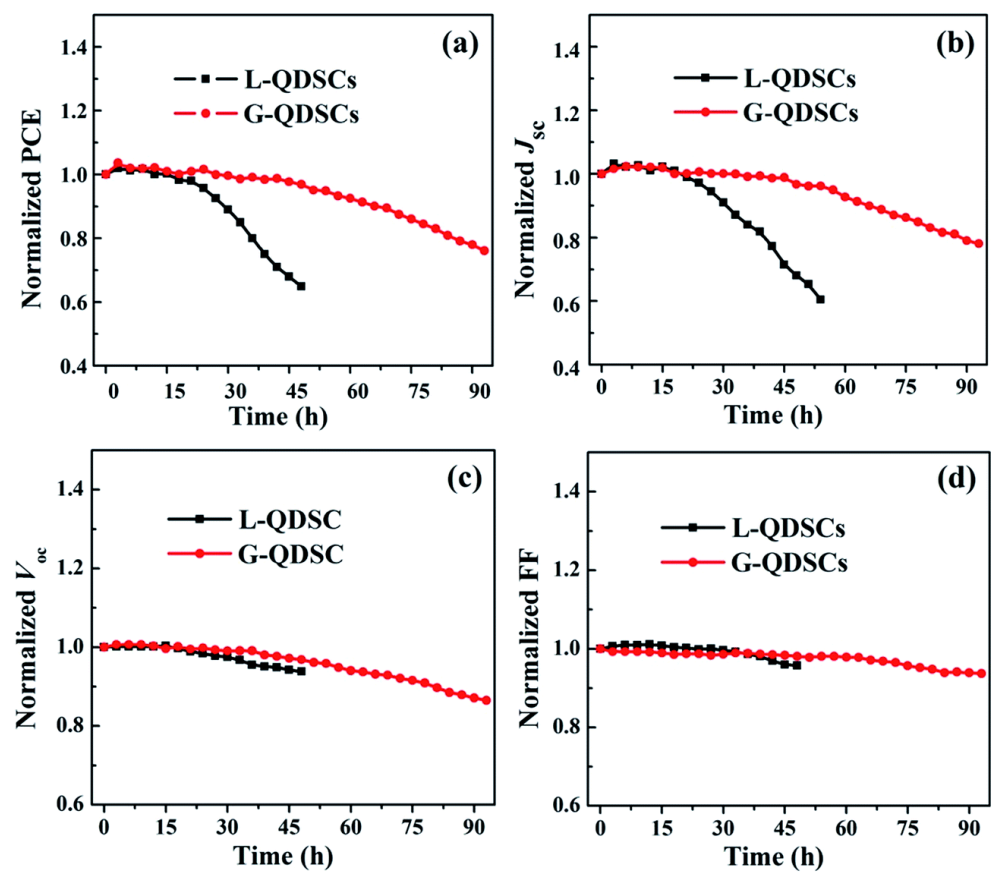

Fig. 37 Normalized performance variation of liquid (L-QDSCs) and gel (G-QDSCs) electrolyte based cells under successive irradiation by an AM 1.5 G solar simulator with an intensity of $100 \mathrm{~mW} \mathrm{~cm} \mathrm{~cm}^{-2}$ under room conditions: (a) PCE; (b) $J_{\mathrm{sc}}$; (c) $V_{\mathrm{oc}}$; (d) FF. Reprinted with permission from ref. 426. Copyright (2016) Royal Society of Chemistry. 
On the whole, despite the stability of QDSCs has been distinctly improved in recent years, it is still far from satisfactory for the practical application or in comparison with other kinds of emerging solar cells. Up to now, the best reported stability test for QDSCs is in a course of $1000 \mathrm{~h}$, while the cells were stored under room light conditions. ${ }^{398,399,425,428}$ Additionally, a standard stability test conditions for QDSCs is still lack now. Although a rigorous standard stability measurement conditions for solar cells was defined by the IEC 61646 protocol, which requires that the cells should be tested under specific damp-heat conditions, including light soaking, thermal cycling and ultraviolet preconditioning, this may be not suitable for some emerging solar cells regarding to the specific characteristics of different materials. The long-term stability has been the main limitation for their industrial application. Even though a series of stability characterizations were reported for QDSCs, they are conducted in non-standardized ways with incomparable data. Meanwhile, a standard stability test conditions for QDSCs is still lack now. Although a rigorous industrial standard stability measurement conditions for commercial photovoltaic panels was defined by the IEC 61646 protocol, stability assessment of a novel solar panel with its own peculiarities might require an adjustment of the common standards. ${ }^{487}$ Therefore, it is still a great challenge to realize a recognized stability and test conditions for QDSCs in the future. 
Table 6 Summary of the stability tests for QDSCs under different test conditions.

\begin{tabular}{|c|c|c|c|c|c|c|}
\hline QDs & $\mathbf{C E}$ & electrolyte & storage condition & period (h) & degradation (\%) & Ref. \\
\hline $\mathrm{CdS} / \mathrm{CdSe}$ & $\mathrm{PbS}-\mathrm{CB} / \mathrm{FTO}$ & polysulfide & $\mathrm{AC}^{\mathrm{a}}$ & 1000 & no & 398 \\
\hline $\mathrm{CdS} / \mathrm{CdSe}$ & $\mathrm{CuInS}_{2}-\mathrm{CB} / \mathrm{FTO}$ & polysulfide & in dark at $\mathrm{AC}$ & 1000 & no & 399 \\
\hline $\mathrm{CdSe}_{\mathrm{x}} \mathrm{Te}_{1-\mathrm{x}}$ & $\mathrm{CuS} / \mathrm{FTO}$ & polysulfide/ $\mathrm{SiO}_{2}$ & in dark at $\mathrm{AC}$ & 1000 & 6.3 & 425 \\
\hline $\mathrm{CdS} / \mathrm{CdSe}$ & $\mathrm{Cu}_{2} \mathrm{~S} /$ brass & gel polysulfide & - & 1000 & 27 & 428 \\
\hline $\mathrm{CdS} / \mathrm{CdSe}$ & $\mathrm{Cu}_{2} \mathrm{~S}$-carbon/FTO & polysulfide & in dark at $\mathrm{AC}$ & 720 & no & 396 \\
\hline CdS/Au@PAA & $\mathrm{C}$-fabric/ $\mathrm{WO}_{3-\mathrm{x}}$ & polysulfide/ $\mathrm{SiO}_{2}$ & in dark at $\mathrm{AC}$ & 720 & 3 & 204 \\
\hline $\mathrm{CdS} / \mathrm{CdSe}$ & $\mathrm{PbSe}$ & {$[\mathrm{DHexBIm}][\mathrm{SCN}]$} & - & 504 & 33 & 420 \\
\hline $\mathrm{CdSe}_{\mathrm{x}} \mathrm{Te}_{1-\mathrm{x}}$ & $\mathrm{Cu}_{2} \mathrm{~S} / \mathrm{FTO}$ & polysulfide & $\mathrm{AC}$ & 500 & no & 111 \\
\hline $\mathrm{CdS} / \mathrm{CdSe}$ & $\mathrm{CuS} / \mathrm{FTO}$ & gel polysulfide & - & 240 & no & 342 \\
\hline $\mathrm{CdS} / \mathrm{CdSe}$ & $\mathrm{Pt}$ & gel polysulfide & in oven at $60^{\circ} \mathrm{C}$ & 220 & 8 & 431 \\
\hline $\mathrm{CdSe}_{\mathrm{x}} \mathrm{Te}_{1-\mathrm{x}}$ & $\mathrm{Cu}_{2} \mathrm{~S} / \mathrm{FTO}$ & polysulfide & $\mathrm{AC}$ & 120 & 20 & 473 \\
\hline $\mathrm{CdSe}_{\mathrm{x}} \mathrm{Te}_{1-\mathrm{x}}$ & $\mathrm{Cu}_{2} \mathrm{~S} / \mathrm{FTO}$ & polysulfide/PVP & $\mathrm{AC}$ & 80 & 15 & 205 \\
\hline $\mathrm{CdSe}$ & $\mathrm{Cu}_{2} \mathrm{~S} / \mathrm{FTO}$ & polysulfide/TEOS & $\mathrm{AC}$ & 76 & 6 & 391 \\
\hline $\mathrm{CdSe}_{\mathrm{x}} \mathrm{Te}_{1-\mathrm{x}}$ & $\mathrm{Cu}_{2} \mathrm{~S} / \mathrm{FTO}$ & gel polysulfide & 1 sun illumination & 90 & 20 & 426 \\
\hline $\mathrm{CdSe}$ & $\mathrm{Cu}_{\mathrm{x}} \mathrm{S} / \mathrm{FTO}$ & gel polysulfide & 1 sun illumination & 77 & 23 & 432 \\
\hline $\mathrm{Sb}_{2} \mathrm{~S}_{3}$ & $\mathrm{Au}$ & $\mathrm{CuSCN}$ & 0.6 sun illumination & 72 & 10 & 441 \\
\hline $\mathrm{CdSe}_{\mathrm{x}} \mathrm{Te}_{1-\mathrm{x}}$ & $\mathrm{Cu}_{\mathrm{x}} \mathrm{S} / \mathrm{FTO}$ & gel polysulfide & 1 sun illumination & 46 & 31 & 432 \\
\hline $\mathrm{CdSe}$ & $\mathrm{Cu}_{\mathrm{x}} \mathrm{S} / \mathrm{FTO}$ & polysulfide/PEG & 1 sun illumination & 30 & no & 424 \\
\hline $\mathrm{CdSe}_{\mathrm{x}} \mathrm{Te}_{1-\mathrm{x}}$ & $\mathrm{Cu}_{2} \mathrm{~S} / \mathrm{FTO}$ & polysulfide & 1 sun illumination & 24 & no & 117 \\
\hline $\mathrm{CdSe}$ & $\mathrm{Cu}_{2} \mathrm{~S} / \mathrm{FTO}$ & polysulfide & 1 sun illumination & 10 & no & 349 \\
\hline $\mathrm{CdS} / \mathrm{CdSe}$ & NiS-PbS/FTO & polysulfide & 1 sun illumination & 10 & no & 411 \\
\hline CdS/Au@PAA & $\mathrm{C}$-fabric/ $/ \mathrm{WO}_{3-\mathrm{x}}$ & polysulfide/ $\mathrm{SiO}_{2}$ & 1-2 sun illumination & 5 & 18 & 204 \\
\hline $\mathrm{Mn}-\mathrm{CdS} / \mathrm{CdSe}$ & $\mathrm{Cu}_{2} \mathrm{~S}-\mathrm{RGO} / \mathrm{FTO}$ & polysulfide & 1 sun illumination & 2 & 2 & 107 \\
\hline
\end{tabular}




\section{Summary and outlook}

In recent years, QDSCs have emerged as an attractive candidate for the third-generation solar cells. During the past five years, we have been witnessing a fast improvement of the obtained record PCEs for QDSCs, arising both from the material and technical promotion. The record PCE is about $13 \%$ now, a competitive level relative to other kind of emerging solar cells. This motivates us to further promote this kind of solar cells to higher level aiming at commercial applications. In this review article, we give a comprehensive overview of the progress, fundamental principle, and main research areas about QDSCs. Finally, we will discuss possible future directions for this field of research with the aim toward highly efficient QDSCs.

To further promote the development of QDSCs, the PCE and stability of the cells together with the toxicity of various constituents should be taken into consideration simultaneously, and improved to a new satisfactory level. Accordingly, the authors believed that the following aspects may deserve great attention in the future:

(a) Developing completely "green" QDSCs. For real application of a new kind of solar cell, the characteristics of environmental friendly of the whole device is requisite. As for QDSCs, the environmental concerns come from various constituents such as the high toxicity from the $\mathrm{Cd}, \mathrm{Pb}$-containing QD materials, the toxicity from the nanoscaled materials, and from the polysulfide redox etc. Therefore, the "green" concept should be taken into account in the study of all components of QDSCs. Concretely, Cd- and Pb-free I-III-VI group QDs such as $\mathrm{CuInS}_{2}$ and $\mathrm{CuInSe}_{2}$ are wise choice as light-absorbing sensitizer in QDSCs. In fact, I-III-VI group QDs have been demonstrated to be capable of obtaining higher efficiency in comparison with $\mathrm{Cd}$ and $\mathrm{Pb}$ based QDs. ${ }^{15,222}$ For counter electrode, carbon materials may be good choice due to their environmental friendly, high catalytic activity, and low-cost. From the safety point of view, QDSCs with aqueous electrolyte are more promising for commercial application in comparison with DSCs with organic electrolyte.

(b) Explore more superior QD sensitizers. The development of QD sensitizers contributed to the huge enhancement of the PCE of QDSCs in recent years. Nevertheless, there is still in great need to explore new type of QD sensitizers, which possess the characteristics of suitable band edge position, wide absorption range, lower density of trap states, environmental friendly and low-cost. Specifically, the construction of composite structured I - III - VI group 
QDs through alloying or core/shell strategy is a promising way for obtaining high quality and low toxicity environmental friendly QD light absorbers in QDSCs.

(c) Improve the loading amount of QDs on $\mathrm{TiO}_{2}$ substrate furthermore. The highest reported surface coverage ratio of QDs on $\mathrm{TiO}_{2}$ electrode is only about $34 \% .{ }^{110}$ It means that large portion of $\mathrm{TiO}_{2}$ electrode surface is bare and uncovered. Further improvement of QDs loading amount can not only improve the light harvesting efficiency, but also inhibit charge recombination. The co-sensitization strategy with use of two or more kinds of QDs to sensitize $\mathrm{TiO}_{2}$ film electrode may be a good choice to further improve the QD loading amount. In addition, the application of solvent engineering to reasonably tailor the polarity of QD solution may also be helpful for enhancing QD loading amount on metal oxide film electrodes.

(d) Suppress charge recombination processes. The severe charge recombination in QDSCs still constitutes one of the main factors that limiting cell performance. It is thereby an urgent need but is still a significant challenge to explore facile and robust method to realize a powerful control of the charge recombination processes, and therefore significantly reduce the energy loss during the charge transfer and transport processes. Up to now, surface coating strategy is commonly used to modify the interfaces, and inhibit charge recombination processes. This coating treatment is mainly carried out through sol-gel route, whereas it's difficult to obtain a compact film on the photoanode surface through the sol-gel approach, resulting in the incomplete passivation effect at interfaces. Therefore, other surface treatment techniques, such as atomic layer deposition (ALD) may provide a more robust passivation layer and thereby improve the photovoltaic performance furthermore.

(e) Explore new kind of electrolyte or HTM. Up to now, the development of electrolyte in QDSCs is still behindhand, thus restricting the $V_{\mathrm{oc}}$ and PCE values of QDSCs. This has become the bottleneck for the further development of QDSCs. It is believed that the breakthrough in electrolyte exploration will dramatically improve the photovoltaic performance of QDSCs. In this regard, we expect that the $\mathrm{Co}^{2+/ 3+}$ complexes redox couple and p-type inorganic semiconductors, such as $\mathrm{CuI}, \mathrm{CuSCN}$, and perovskite materials, are promising candidates as electrolytes or HTMs in QDSCs.

(f) Realize long-term stability of the device. The current stability of QDSCs is still far from the standard of real application. Further effort is required to improve the long-term stability of QDSCs. The improvement of the device sealing technique for the liquid junction QDSCs, 
or fabrication all inorganic solid-state QDSCs possess potential to realize the satisfactory long-term stability. Additionally, a standard stability test conditions for QDSCs is also needed to reasonably evaluate the stability of the device.

(g) Concerning the commercialization of this technology QDSCs can take advantage of the developments made in this line by DSCs. ${ }^{498}$ No technical difficulty to make upscaling and fabricate large modules for QDSCs should be expected since its analogue DSCs succeeded in these fields. As for DSCs long term stability is the major issue for QDSCs. Unfortunately, as far as we know there are no specific works focusing on the life cycle assessment of QDSCs to evaluate the cost and the environmental impacts produced by the upscaling of this technology in order to determine the main limiting factors in terms of cost, technology, toxicity or stability, and this lack needs to solved in the future.

The fascinating advantages combined with the fast evolution of QDSC render it a promising candidate for the next-generation solar cell. We are confident that with further breakthroughs in materials exploring and device structure optimization, QDSC with a PCE over $15 \%$ will be obtained in the near future. 


\section{Conflicts of interest}

There are no conflicts of interest to declare.

\section{Acknowledgements}

This research is supported by the National Natural Science Foundation of China (Nos. 51732004, 21703071, 91433106). 


\section{References}

1. R. E. Smalley, Abstr. Paper Am. Chem. Soc., 2003, 226, U24.

2. O. Morton, Nature, 2006, 443, 19-22.

3. N. S. Lewis and D. G. Nocera, Proc. Natl. Acad. Sci. U. S. A., 2006, 103, 15729-15735.

4. A. Hagfeldt, G. Boschloo, L. Sun, L. Kloo and H. Pettersson, Chem. Rev., 2010, 110, 6595-6663.

5. D. Chapin, C. Fuller and G. Pearson, J. Appl. Phys., 1954, 25, 676.

6. J. Wu, Z. Lan, J. Lin, M. Huang, Y. Huang, L. Fan and G. Luo, Chem. Rev., 2015, 115, 21362173.

7. J. Wu, Z. Lan, J. Lin, M. Huang, Y. Huang, L. Fan, G. Luo, Y. Lin, Y. Xie and Y. Wei, Chem. Soc. Rev., 2017, 46, 5975-6023.

8. C. Battaglia, A. Cuevas and S. De Wolf, Energy Environ. Sci., 2016, 9, 1552-1576.

9. T. Saga, NPG Asia Mater., 2010, 2, 96-102.

10. J. K. Rath, Sol. Energy Mater. Sol. Cells, 2003, 76, 431-487.

11. P. Jackson, D. Hariskos, E. Lotter, S. Paetel, R. Wuerz, R. Menner, W. Wischmann and M. Powalla, Prog. Photovoltaics, 2011, 19, 894-897.

12. K. Nakayama, K. Tanabe and H. A. Atwater, Appl. Phys. Lett., 2008, 93, 121904.

13. F. Dimroth, M. Grave, P. Beutel, U. Fiedeler, C. Karcher, T. N. D. Tibbits, E. Oliva, G. Siefer, M. Schachtner, A. Wekkeli, A. W. Bett, R. Krause, M. Piccin, N. Blanc, C. Drazek, E. Guiot, B. Ghyselen, T. Salvetat, A. Tauzin, T. Signamarcheix, A. Dobrich, T. Hannappel and K. Schwarzburg, Prog. Photovoltaics, 2014, 22, 277-282.

14. A. Romeo, A. Terheggen, D. Abou-Ras, D. L. Batzner, F. J. Haug, M. Kalin, D. Rudmann and A. N. Tiwari, Prog. Photovoltaics, 2004, 12, 93-111.

15. X. Z. Wu, Sol. Energy, 2004, 77, 803-814.

16. M. Graetzel, Acc. Chem. Res., 2009, 42, 1788-1798.

17. Y.-J. Cheng, S.-H. Yang and C.-S. Hsu, Chem. Rev., 2009, 109, 5868-5923.

18. S. Guenes, H. Neugebauer and N. S. Sariciftci, Chem. Rev., 2007, 107, 1324-1338.

19. G. H. Carey, A. L. Abdelhady, Z. Ning, S. M. Thon, O. M. Bakr and E. H. Sargent, Chem. Rev., 2015, 115, 12732-12763.

20. A. J. Nozik, M. C. Beard, J. M. Luther, M. Law, R. J. Ellingson and J. C. Johnson, Chem. Rev, 2010, 110, 6873-6890.

21. A. Kojima, K. Teshima, Y. Shirai and T. Miyasaka, J. Am. Chem. Soc., 2009, 131, 6050-6051.

22. G. Hodes, Science, 2013, 342, 317-318.

23. M. M. Lee, J. Teuscher, T. Miyasaka, T. N. Murakami and H. J. Snaith, Science, 2012, 338, 643647.

24. M. Kouhnavard, S. Ikeda, N. A. Ludin, N. B. Ahmad Khairudin, B. V. Ghaffari, M. A. Mat-Teridi, M. A. Ibrahim, S. Sepeai and K. Sopian, Renew. Sust. Energy Rev., 2014, 37, 397-407.

25. I. J. Kramer and E. H. Sargent, ACS Nano, 2011, 5, 8506-8514.

26. P. V. Kamat, K. Tvrdy, D. R. Baker and J. G. Radich, Chem. Rev., 2010, 110, 6664-6688.

27. I. J. Kramer and E. H. Sargent, Chem. Rev., 2014, 114, 863-882.

28. O. E. Semonin, J. M. Luther, S. Choi, H.-Y. Chen, J. Gao, A. J. Nozik and M. C. Beard, Science, 2011, 334, 1530-1533.

29. M. R. Kim and D. Ma, J. Phys. Chem. Lett., 2015, 6, 85-99.

30. S. Ruhle, M. Shalom and A. Zaban, ChemPhysChem, 2010, 11, 2290-2304. 
31. S. Emin, S. P. Singh, L. Han, N. Satoh and A. Islam, Sol. Energy, 2011, 85, 1264-1282.

32. P. V. Kamat, Acc. Chem. Res., 2012, 45, 1906-1915.

33. I. Mora-Sero, S. Gimenez, F. Fabregat-Santiago, R. Gomez, Q. Shen, T. Toyoda and J. Bisquert, Acc. Chem. Res., 2009, 42, 1848-1857.

34. J. Duan, H. Zhang, Q. Tang, B. He and L. Yu, J. Mater. Chem. A, 2015, 3, 17497-17510.

35. P. V. Kamat, J. Phys. Chem. Lett., 2013, 4, 908-918.

36. W. Li and X. Zhong, J. Phys. Chem. Lett., 2015, 6, 796-806.

37. I. Mora-Sero and J. Bisquert, J. Phys. Chem. Lett., 2010, 1, 3046-3052.

38. K. Zhao, Z. Pan and X. Zhong, J. Phys. Chem. Lett., 2016, 7, 406-417.

39. P. V. Kamat, J. A. Christians and J. G. Radich, Langmuir, 2014, 30, 5716-5725.

40. J.-K. Sun, Y. Jiang, X. Zhong, J.-S. Hu and L.-J. Wan, Nano Energy, 2017, 32, 130-156.

41. S. Kumar, M. Nehra, A. Deep, D. Kedia, N. Dilbaghi and K.-H. Kim, Renew. Sust. Energy Rev., 2017, 73, 821-839.

42. M. Ye, X. Gao, X. Hong, Q. Liu, C. He, X. Liu and C. Lin, Sustainable Energy Fuels., 2017, 1, 1217-1231.

43. G. Hodes, J. Phys. Chem. C, 2008, 112, 17778-17787.

44. D. F. Watson, J. Phys. Chem. Lett., 2010, 1, 2299-2309.

45. Z. Yang, C. Y. Chen, P. Roy and H. T. Chang, Chem. Commun., 2011, 47, 9561-9571.

46. I. Hod and A. Zaban, Langmuir, 2014, 30, 7264-7273.

47. J. Xu, Z. Chen, J. A. Zapien, C. S. Lee and W. Zhang, Adv. Mater., 2014, 26, 5337-5367.

48. I. Hwang and K. Yong, ChemElectroChem, 2015, 2, 634-653.

49. K. Meng, G. Chen and K. R. Thampi, J. Mater. Chem. A, 2015, 3, 23074-23089.

50. J. Tian and G. Cao, J. Phys. Chem. Lett., 2015, 6, 1859-1869.

51. M. Wu, X. Lin, Y. Wang and T. Ma, J. Mater. Chem. A, 2015, 3, 19638-19656.

52. C. Shen, D. Fichou and Q. Wang, Chem. Asian J., 2016, 11, 1183-1193.

53. R. Xia, S.-M. Wang, W.-W. Dong and X.-D. Fang, Acta Phys-Chim. Sin., 2017, 33, 670-690.

54. H. Gerischer, M. E. Michel-Beyerle, F. Rebentrost and H. Tributsch, Electrochim. Acta, 1968, 13, $1509-1515$.

55. K. Kalyanasundaram, Sol. Cells, 1985, 15, 93-156.

56. B. O'Regan and M. Gratzel, Nature, 1991, 353, 737-740.

57. P. Wang, S. M. Zakeeruddin, J. E. Moser, M. K. Nazeeruddin, T. Sekiguchi and M. Gratzel, Nat. Materials, 2003, 2, 402-407.

58. M. Law, L. E. Greene, J. C. Johnson, R. Saykally and P. D. Yang, Nat. Materials, 2005, 4, 455459.

59. X. Wang, L. Zhi and K. Muellen, Nano Lett., 2008, 8, 323-327.

60. A. Mishra, M. K. R. Fischer and P. Baeuerle, Angew. Chem. Int. Ed., 2009, 48, 2474-2499.

61. A. Yella, H.-W. Lee, H. N. Tsao, C. Yi, A. K. Chandiran, M. K. Nazeeruddin, E. W.-G. Diau, C.-Y. Yeh, S. M. Zakeeruddin and M. Graetzel, Science, 2011, 334, 629-634.

62. S. Mathew, A. Yella, P. Gao, R. Humphry-Baker, B. F. E. Curchod, N. Ashari-Astani, I. Tavernelli, U. Rothlisberger, M. K. Nazeeruddin and M. Graetzel, Nat. Chemistry, 2014, 6, 242-247.

63. J. Bisquert, CRC Press, 2017, Boca Raton.

64. P. V. Kamat, J. Phys. Chem. C, 2008, 112, 18737-18753.

65. M. Pazoki, U. B. Cappel, E. M. J. Johansson, A. Hagfeldt and G. Boschloo, Energy Environ. Sci., 2017, 10, 672-709. 
66. F. Fabregat-Santiago, G. Garcia-Belmonte, I. Mora-Sero and J. Bisquert, Phys. Chem. Chem. Phys., 2011, 13, 9083-9118.

67. K. Zheng, K. Zidek, M. Abdellah, P. Chabera, M. S. Abd El-Sadek and T. Pullerits, Appl. Phys. Lett., 2013, 102, 163119.

68. K. Zidek, K. Zheng, P. Chabera, M. Abdellah and T. Pullerits, Appl. Phys. Lett., 2012, 100, 243111.

69. K. Zidek, K. Zheng, C. S. Ponseca, Jr., M. E. Messing, L. R. Wallenberg, P. Chabera, M. Abdellah, V. Sundstrom and T. Pullerits, J. Am. Chem. Soc., 2012, 134, 12110-12117.

70. M. Abdellah, R. Marschan, K. Zidek, M. E. Messing, A. Abdelwahab, P. Chabera, K. Zheng and T. Pullerits, J. Phys. Chem. C, 2014, 118, 25802-25808.

71. K. Zheng, K. Zidek, M. Abdellah, W. Zhang, P. Chabera, N. Lenngren, A. Yartsev and T. Pullerits, J. Phys. Chem. C, 2014, 118, 18462-18471.

72. K. Zidek, K. Zheng, M. Abdellah, N. Lenngren, P. Chabera and T. Pullerits, Nano Lett., 2012, 12, 6393-6399.

73. A. Kongkanand, K. Tvrdy, K. Takechi, M. Kuno and P. V. Kamat, J. Am. Chem. Soc., 2008, 130, 4007-4015.

74. J. Wang, I. Mora-Sero, Z. Pan, K. Zhao, H. Zhang, Y. Feng, G. Yang, X. Zhong and J. Bisquert, J. Am. Chem. Soc., 2013, 135, 15913-15922.

75. V. Chakrapani, D. Baker and P. V. Kamat, J. Am. Chem. Soc., 2011, 133, 9607-9615.

76. K. Tvrdy, P. A. Frantsuzov and P. V. Kamat, Proc. Natl. Acad. Sci. USA, 2011, 108, 29-34.

77. P. K. Santra, P. V. Nair, K. G. Thomas and P. V. Kamat, J. Phys. Chem. Lett., 2013, 4, 722-729.

78. H. McDaniel, N. Fuke, N. S. Makarov, J. M. Pietryga and V. I. Klimov, Nat. Commun., 2013, 4, 2887.

79. H. McDaniel, N. Fuke, J. M. Pietryga and V. I. Klimov, J. Phys. Chem. Lett., 2013, 4, 355-361.

80. D. R. Pernik, K. Tvrdy, J. G. Radich and P. V. Kamat, J. Phys. Chem. C, 2011, 115, 13511-13519.

81. V. Gonzalez-Pedro, X. Xu, I. Mora-Sero and J. Bisquert, ACS Nano, 2010, 4, 5783-5790.

82. A. Zaban, M. Greenshtein and J. Bisquert, ChemPhysChem, 2003, 4, 859-864.

83. J. Bisquert, F. Fabregat-Santiago, I. Mora-Sero, G. Garcia-Belmonte and S. Gimenez, J. Phys. Chem. C, 2009, 113, 17278-17290.

84. J. Fan, L. Li, H.-S. Rao, Q.-L. Yang, J. Zhang, H.-Y. Chen, L. Chen, D.-B. Kuang and C.-Y. Su, J. Mater. Chem. A, 2014, 2, 15406-15413.

85. S. Jiao, Q. Shen, I. Mora-Sero, J. Wang, Z. Pan, K. Zhao, Y. Kuga, X. Zhong and J. Bisquert, ACS Nano, 2015, 9, 908-915.

86. A. Salant, M. Shalom, Z. Tachan, S. Buhbut, A. Zaban and U. Banin, Nano Lett., 2012, 12, 20952100.

87. N. Serpone, E. Borgarello and M. Grätzel, J. Chem. Soc., Chem. Commun., 1984, 0, 342-344.

88. H. Gerischer and M. Lubke, J. Electroanal. Chem., 1986, 204, 225-227.

89. L. Spanhel, H. Weller and A. Henglein, J. Am. Chem. Soc., 1987, 109, 6632-6635.

90. K. R. Gopidas, M. Bohorquez and P. V. Kamat, J. Phys. Chem., 1990, 94, 6435-6440.

91. R. Vogel, K. Pohl and H. Weller, Chem. Phys. Lett., 1990, 174, 241-246.

92. S. Kohtani, A. Kudo and T. Sakata, Chem. Phys. Lett., 1993, 206, 166-170.

93. D. Liu and P. V. Kamat, J. Phys. Chem., 1993, 97, 10769-10773.

94. R. Vogel, P. Hoyer and H. Weller, J. Phys. Chem., 1994, 98, 3183-3188.

95. A. Zaban, O. I. Micic, B. A. Gregg and A. J. Nozik, Langmuir, 1998, 14, 3153-3156. 
96. P. Yu, K. Zhu, A. G. Norman, S. Ferrere, A. J. Frank and A. J. Nozik, J. Phys. Chem. B, 2006, 110, 25451-25454.

97. R. Plass, S. Pelet, J. Krueger, M. Grätzel and U. Bach, J. Phys. Chem. B, 2002, 106, 7578-7580.

98. A. J. Nozik, Phys. E, 2002, 14, 115-120.

99. L. J. Diguna, Q. Shen, J. Kobayashi and T. Toyoda, Appl. Phys. Lett., 2007, 91, 023116.

100. Y.-L. Lee, B.-M. Huang and H.-T. Chien, Chem. Mater., 2008, 20, 6903-6905.

101. Y.-L. Lee and Y.-S. Lo, Adv. Funct. Mater., 2009, 19, 604-609.

102. H. Lee, M. Wang, P. Chen, D. R. Gamelin, S. M. Zakeeruddin, M. Graetzel and M. K. Nazeeruddin, Nano Lett., 2009, 9, 4221-4227.

103. S.-Q. Fan, B. Fang, J. H. Kim, B. Jeong, C. Kim, J.-S. Yu and J. Ko, Langmuir, 2010, 26, 13644 13649.

104. B. Fang, M. Kim, S.-Q. Fan, J. H. Kim, D. P. Wilkinson, J. Ko and J.-S. Yu, J. Mater. Chem., 2011, 21, 8742-8748.

105. J. Xu, X. Yang, H. Wang, X. Chen, C. Luan, Z. Xu, Z. Lu, V. A. Roy, W. Zhang and C. S. Lee, Nano Lett., 2011, 11, 4138-4143.

106. Q. Zhang, X. Guo, X. Huang, S. Huang, D. Li, Y. Luo, Q. Shen, T. Toyoda and Q. Meng, Phys. Chem. Chem. Phys., 2011, 13, 4659-4667.

107. P. K. Santra and P. V. Kamat, J. Am. Chem. Soc., 2012, 134, 2508-2511.

108.E. Martinez-Ferrero, I. M. Sero, J. Albero, S. Gimenez, J. Bisquert and E. Palomares, Phys. Chem. Chem. Phys., 2010, 12, 2819-2821.

109. Z. Pan, H. Zhang, K. Cheng, Y. Hou, J. Hua and X. Zhong, ACS Nano, 2012, 6, 3982-3991.

110. H. Zhang, K. Cheng, Y. M. Hou, Z. Fang, Z. X. Pan, W. J. Wu, J. L. Hua and X. H. Zhong, Chem. Commun., 2012, 48, 11235-11237.

111. Z. Pan, K. Zhao, J. Wang, H. Zhang, Y. Feng and X. Zhong, ACS Nano, 2013, 7, 5215-5222.

112. W. Wang, W. Feng, J. Du, W. Xue, L. Zhang, L. Zhao, Y. Li and X. Zhong, Adv. Mater., 2018, 30, 1705746.

113. Z. Ren, J. Wang, Z. Pan, K. Zhao, H. Zhang, Y. Li, Y. Zhao, I. Mora-Sero, J. Bisquert and X. Zhong, Chem. Mater., 2015, 27, 8398-8405.

114. Z. Ren, Z. Wang, R. Wang, Z. Pan, X. Gong and X. Zhong, Chem. Mater, 2016, 28, 2323-2330.

115. J. Du, Z. Du, J. S. Hu, Z. Pan, Q. Shen, J. Sun, D. Long, H. Dong, L. Sun, X. Zhong and L. J. Wan, J. Am. Chem. Soc., 2016, 138, 4201-4209.

116. Z. Pan, I. Mora-Sero, Q. Shen, H. Zhang, Y. Li, K. Zhao, J. Wang, X. Zhong and J. Bisquert, J. Am. Chem. Soc., 2014, 136, 9203-9210.

117. K. Zhao, Z. Pan, I. Mora-Sero, E. Canovas, H. Wang, Y. Song, X. Gong, J. Wang, M. Bonn, J. Bisquert and X. Zhong, J. Am. Chem. Soc., 2015, 137, 5602-5609.

118. Z. Du, Z. Pan, F. Fabregat-Santiago, K. Zhao, D. Long, H. Zhang, Y. Zhao, X. Zhong, J. S. Yu and J. Bisquert, J. Phys. Chem. Lett., 2016, 7, 3103-3111.

119. S. Jiao, J. Du, Z. Du, D. Long, W. Jiang, Z. Pan, Y. Li and X. Zhong, J. Phys. Chem. Lett., 2017, 8, 559-564.

120. Y. Bai, I. Mora-Sero, F. De Angelis, J. Bisquert and P. Wang, Chem. Rev., 2014, 114, 1009510130.

121. J. Zhang, J. H. Bang, C. Tang and P. V. Kamat, ACS Nano, 2010, 4, 387-395.

122. Q. Shen, A. Yamada, S. Tamura and T. Toyoda, Appl. Phys. Lett., 2010, 97, 123107.

123. H. Han, P. Sudhagar, T. Song, Y. Jeon, I. Mora-Sero, F. Fabregat-Santiago, J. Bisquert, Y. S. Kang 
and U. Paik, Chem. Commun., 2013, 49, 2810-2812.

124. J. Zhang, C. Tang and J. H. Bang, Electrochem. Commun., 2010, 12, 1124-1128.

125.B. Liu, Y. Sun, X. Wang, L. Zhang, D. Wang, Z. Fu, Y. Lin and T. Xie, J. Mater. Chem. A, 2015, 3, 4445-4452.

126. C. Dong, X. Li and J. Qi, J. Phys. Chem. C, 2011, 115, 20307-20315.

127. L. Tao, Y. Xiong, H. Liu and W. Shen, Nanoscale, 2014, 6, 931-938.

128. S. Huang, Q. Zhang, X. Huang, X. Guo, M. Deng, D. Li, Y. Luo, Q. Shen, T. Toyoda and Q. Meng, Nanotechnology, 2010, 21, 375201.

129. W. Lee, S. H. Kang, J.-Y. Kim, G. B. Kolekar, Y.-E. Sung and S.-H. Han, Nanotechnology, 2009, 20, 335706.

130. M. Akimoto, T. Toyoda, T. Okuno, S. Hayase and Q. Shen, Thin Solid Films, 2015, 590, 90-97.

131. H. Huang, L. Pan, C. K. Lim, H. Gong, J. Guo, M. S. Tse and O. K. Tan, Small, 2013, 9, $3153-$ 3160.

132. L. Yu, Z. Li, Y. Liu, F. Cheng and S. Sun, Appl. Surf. Sci., 2014, 309, 255-262.

133. Z. Zhang, C. Shi, J. Chen, G. Xiao and L. Li, Appl. Surf. Sci., 2017, 410, 8-13.

134. Z. Zhang, C. Shi, G. Xiao, K. Lv, C. Ma and J. Yue, Ceram. Int., 2017, 43, 10052-10056.

135. Y. Chen, Q. Tao, W. Fu, H. Yang, X. Zhou, S. Su, D. Ding, Y. Mu, X. Li and M. Li, Chem. Commun., 2014, 50, 9509-9512.

136. C. Chen, M. Ye, M. Lv, C. Gong, W. Guo and C. Lin, Electrochim. Acta, 2014, 121, 175-182.

137. R. Zhou, Y. Huang, L. Wan, H. Niu, F. Ji and J. Xu, J. Alloy. Comp., 2017, 716, 162-170.

138. C. Wang, Z. Jiang, L. Wei, Y. Chen, J. Jiao, M. Eastman and H. Liu, Nano Energy, 2012, 1, 440447.

139. J. Wan, R. Liu, Y. Tong, S. Chen, Y. Hu, B. Wang, Y. Xu and H. Wang, Nanoscale Res. Lett., 2016, 11, 12.

140. W. Zhang, X. Zeng, H. Wang, R. Fang, Y. Xu, Y. Zhang and W. Chen, RSC Adv., 2016, 6, $33713-33722$.

141. Z. Peng, Y. Liu, Y. Zhao, K. Chen, Y. Cheng and W. Chen, Electrochim. Acta, 2014, 135, 276283.

142. L. Yu, X. Ren, Z. Yang, Y. Han and Z. Li, J. Mater. Sci-Mater. El., 2016, 27, 7150-7160.

143. A. Fatehmulla, M. A. Manthrammel, W. A. Farooq, S. M. Ali and M. Atif, J. Nanomater, 2015, 2015, 358063.

144. H.-S. Rao, W.-Q. Wu, Y. Liu, Y.-F. Xu, B.-X. Chen, H.-Y. Chen, D.-B. Kuang and C.-Y. Su, Nano Energy, 2014, 8, 1-8.

145. Y.-F. Xu, W.-Q. Wu, H.-S. Rao, H.-Y. Chen, D.-B. Kuang and C.-Y. Su, Nano Energy, 2015, 11, 621-630.

146. D. R. Baker and P. V. Kamat, Adv. Funct. Mater., 2009, 19, 805-811.

147. Z. Zhang, C. Shi, J. Chen, G. Xiao and L. Li, Applied Surface Science, 2017, 410, 8-13.

148. H. Zhou, L. Li, D. Jiang, Y. Lu and K. Pan, RSC Adv., 2016, 6, 67968-67975.

149. C. Li, L. Yang, J. Xiao, Y.-C. Wu, M. Sondergaard, Y. Luo, D. Li, Q. Meng and B. B. Iversen, Phys. Chem. Chem. Phys., 2013, 15, 8710-8715.

150. J. Xu, X. Yang, Q.-D. Yang, T.-L. Wong, S.-T. Lee, W.-J. Zhang and C.-S. Lee, J. Mater. Chem., 2012, 22, 13374-13379.

151. D. Wu, X. Wang, K. Cao, Y. An, X. Song, N. Liu, F. Xu, Z. Gao and K. Jiang, Electrochim. Acta, 2017, 231, 1-12. 
152. K. Yan, L. Zhang, J. Qiu, Y. Qiu, Z. Zhu, J. Wang and S. Yang, J. Am. Chem. Soc., 2013, 135, 9531-9539.

153. J. Xiao, Q. Huang, J. Xu, C. Li, G. Chen, Y. Luo, D. Li and Q. Meng, J. Phys. Chem. C, 2014, 118, 4007-4015.

154. M. Raissi, Y. Pellegrin, S. Jobic, M. Boujtita and F. Odobel, Sci. Rep., 2016, 6, 24908.

155. H.-M. Cheng, K.-Y. Huang, K.-M. Lee, P. Yu, S.-C. Lin, J.-H. Huang, C.-G. Wu and J. Tang, Phys. Chem. Chem. Phys., 2012, 14, 13539-13548.

156. C. J. Raj, S. N. Karthick, K. V. Hemalatha, H.-J. Kim and K. Prabakar, Thin Solid Films, 2013, 548, 636-640.

157. J. Deng, M. Wang, C. Yang, J. Liu and X. Song, RSC Adv., 2014, 4, 41141-41147.

158. F. S. Ghoreishi, V. Ahmadi and M. Samadpour, J. Power Sources, 2014, 271, 195-202.

159. M.-S. Jeong, M.-K. Son, S.-K. Kim, S. Park, K. Prabakar and H.-J. Kim, Thin Solid Films, 2014, 570, 310-314.

160. D. Karageorgopoulos, E. Stathatos and E. Vitoratos, J. Power Sources, 2012, 219, 9-15.

161. J. Tian, Q. Zhang, E. Uchaker, R. Gao, X. Qu, S. Zhang and G. Cao, Energy Environ. Sci., 2013, 6, 3542-3547.

162. J. Tian, L. Lv, X. Wang, C. Fei, X. Liu, Z. Zhao, Y. Wang and G. Cao, J. Phys. Chem. C, 2014, 118, 16611-16617.

163. K. S. Leschkies, R. Divakar, J. Basu, E. Enache-Pommer, J. E. Boercker, C. B. Carter, U. R. Kortshagen, D. J. Norris and E. S. Aydil, Nano Lett., 2007, 7, 1793-1798.

164. M. Seol, E. Ramasamy, J. Lee and K. Yong, J. Phys. Chem. C, 2011, 115, 22018-22024.

165. P. Sudhagar, T. Song, D. H. Lee, I. Mora-Sero, J. Bisquert, M. Laudenslager, W. M. Sigmund, W.

I. Park, U. Paik and Y. S. Kang, J. Phys. Chem. Lett., 2011, 2, 1984-1990.

166. H. Chen, L. Zhu, H. Liu and W. Li, Nanotechnology, 2012, 23, 075402.

167. R. Zhang, Q.-P. Luo, H.-Y. Chen, X.-Y. Yu, D.-B. Kuang and C.-Y. Su, ChemPhysChem, 2012, 13, 1435-1439.

168. C.-Y. Chou, C.-T. Li, C.-P. Lee, L.-Y. Lin, M.-H. Yeh, R. Vittal and K.-C. Ho, Electrochim. Acta, 2013, 88, 35-43.

169. I. Hwang and K. Yong, ChemPhysChem, 2013, 14, 364-368.

170. H. Kim, H. Jeong, T. K. An, C. E. Park and K. Yong, ACS Appl. Mater. Interfaces, 2013, 5, $268-$ 275.

171. X. Yin, W. Que, D. Fei, H. Xie and Z. He, Electrochim. Acta, 2013, 99, 204-210.

172. X. Yin, W. Que, D. Fei, H. Xie, Z. He and G. Wang, Electrochim. Acta, 2013, 89, 561-570.

173. M.-L. Zhang, F. Jin, M.-L. Zheng, J. Liu, Z.-S. Zhao and X.-M. Duan, RSC Adv., 2014, 4, $10462-$ 10466.

174. W. Lee, S. Kang, T. Hwang, K. Kim, H. Woo, B. Lee, J. Kim, J. Kim and B. Park, Electrochim. Acta, 2015, 167, 194-200.

175. M. Seol, H. Kim, Y. Tak and K. Yong, Chem. Commun., 2010, 46, 5521-5523.

176. H. Chen, W. Li, Q. Hou, H. Liu and L. Zhu, Electrochim. Acta, 2011, 56, 8358-8364.

177. J. Chen, W. Lei, C. Li, Y. Zhang, Y. Cui, B. Wang and W. Deng, Phys. Chem. Chem. Phys., 2011, 13, 13182-13184.

178. J. Chen, C. Li, G. Eda, Y. Zhang, W. Lei, M. Chhowalla, W. I. Milne and W.-Q. Deng, Chem. Commun., 2011, 47, 6084-6086.

179. C.-Z. Yao, B.-H. Wei, L.-X. Meng, H. Li, Q.-J. Gong, H. Sun, H.-X. Ma and X.-H. Hu, J. Power 
Sources, 2012, 207, 222-228.

180. L.-B. Li, Y.-F. Wang, H.-S. Rao, W.-Q. Wu, K.-N. Li, C.-Y. Su and D.-B. Kuang, ACS Appl. Mater. Interfaces, 2013, 5, 11865-11871.

181. J. Tian, Q. Zhang, E. Uchaker, Z. Liang, R. Gao, X. Qu, S. Zhang and G. Cao, J. Mater. Chem. A, 2013, 1, 6770-6775.

182. C. J. Raj, S. N. Karthick, S. Park, K. V. Hemalatha, S.-K. Kim, K. Prabakar and H.-J. Kim, J. Power Sources, 2014, 248, 439-446.

183. J. Tian, E. Uchaker, Q. Zhang and G. Cao, ACS Appl. Mater. Interfaces, 2014, 6, 4466-4472.

184. C. V. V. M. Gopi, M. Venkata-Haritha, Y.-S. Lee and H.-J. Kim, J. Mater. Chem. A, 2016, 4, 8161-8171.

185. J. Hou, H. Zhao, F. Huang, Q. Jing, H. Cao, Q. Wu, S. Peng and G. Cao, J. Power Sources, 2016, 325, 438-445.

186. C. V. V. M. Gopi, M. Venkata-Haritha, Y.-S. Lee and H.-J. Kim, J. Mater. Chem. A, 2017, 5, 428429.

187. Z. Zhu, J. Qiu, K. Yan and S. Yang, ACS Appl. Mater. Interfaces, 2013, 5, 4000-4005.

188. K. Yan, L. Zhang, J. Qiu, Y. Qiu, Z. Zhu, J. Wang and S. Yang, J. Am. Chem. Soc., 2013, 135, 9531-9539.

189. V. Ganapathy, E.-H. Kong, Y.-C. Park, H. M. Jang and S.-W. Rhee, Nanoscale, 2014, 6, 32963301.

190. S. Greenwald, S. Ruhle, M. Shalom, S. Yahav and A. Zaban, Phys. Chem. Chem. Phys., 2011, 13, 19302-19306.

191. A. Pimachev, U. Poudyal, V. Proshchenko, W. Wang and Y. Dahnovsky, Phys. Chem. Chem. Phys., 2016, 18, 26771-26776.

192. J. G. Radich, N. R. Peeples, P. K. Santra and P. V. Kamat, J. Phys. Chem. C, 2014, 118, $16463-$ 16471.

193. J. Park, M. T. Sajjad, P.-H. Jouneau, A. Ruseckas, J. Faure-Vincent, I. D. W. Samuel, P. Reiss and D. Aldakov, J. Mater. Chem. A, 2016, 4, 827-837.

194. M. T. Sajjad, J. Park, D. Gaboriau, J. R. Harwell, F. Odobel, P. Reiss, I. D. W. Samuel and D. Aldakov, J. Phys. Chem. C, 2018, 122, 5161-5170.

195. M. Raissi, M. T. Sajjad, Y. Pellegrin, T. J. Roland, S. Jobic, M. Boujtita, A. Ruseckas, I. D. W. Samuel and F. Odobel, Nanoscale, 2017, 9, 15566-15575.

196. F. Odobel and Y. Pellegrin, J. Phys. Chem. Lett., 2013, 4, 2551-2564.

197. J. H. Rhee, Y. H. Lee, P. Bera and S. I. Seok, Chem. Phys. Lett., 2009, 477, 345-348.

198. T. J. Macdonald, Y. J. Mange, M. R. Dewi, H. U. Islam, I. P. Parkin, W. M. Skinner and T. Nann, J. Mater. Chem. A, 2015, 3, 13324-13331.

199. Y.-Q. Mao, Z.-J. Zhou, T. Ling and X.-W. Du, RSC Adv., 2013, 3, 1217-1221.

200. M. A. Becker, J. G. Radich, B. A. Bunker and P. V. Kamat, J. Phys. Chem. Lett., 2014, 5, 15751582.

201. O. Niitsoo, S. K. Sarkar, C. Pejoux, S. Ruhle, D. Cahen and G. Hodes, J. Photochem. Photobiol. $A, 2006, \mathbf{1 8 1}, 306-313$.

202. C.-H. Chang and Y.-L. Lee, Appl. Phys. Lett., 2007, 91, 053503.

203. J. Tian, L. Lv, C. Fei, Y. Wang, X. Liu and G. Cao, J. Mater. Chem. A, 2014, 2, 19653-19659.

204. P. N. Kumar, A. Kolay, M. Deepa, S. M. Shivaprasad and A. K. Srivastava, ACS Appl. Mater. Interfaces, 2017, 9, 25278 - 25290. 
205. G. Jiang, Z. Pan, Z. Ren, J. Du, C. Yang, W. Wang and X. Zhong, J. Mater. Chem. A, 2016, 4, 11416-11421.

206. J. Yang and X. Zhong, J. Mater. Chem. A, 2016, 4, 16553-16561.

207. J.-W. Lee, D.-Y. Son, T. K. Ahn, H.-W. Shin, I. Y. Kim, S.-J. Hwang, M. J. Ko, S. Sul, H. Han and N.-G. Park, Sci. Rep., 2013, 3, 1050.

208. Y. C. Choi, D. U. Lee, J. H. Noh, E. K. Kim and S. I. Seok, Adv. Funct. Mater, 2014, 24, $3587-$ 3592.

209. S. Yang, P. Zhao, X. Zhao, L. Qu and X. Lai, J. Mater. Chem. A, 2015, 3, 21922-21929.

210. R. K. Chava and M. Kang, Mater. Lett., 2017, 199, 188-191.

211. Y.-S. Chen, H. Choi and P. V. Kamat, J. Am. Chem. Soc., 2013, 135, 8822-8825.

212. Q. Zhang, G. Zhang, X. Sun, K. Yin and H. Li, Nanomaterials, 2017, 7, 130.

213. S. Jiao, J. Wang, Q. Shen, Y. Li and X. Zhong, J. Mater. Chem. A, 2016, 4, 7214-7221.

214. F. Huang, L. Zhang, Q. Zhang, J. Hou, H. Wang, H. Wang, S. Peng, J. Liu and G. Cao, ACS Appl. Mater. Interfaces, 2016, 8, 34482-34489.

215. X.-Y. Yu, B.-X. Lei, D.-B. Kuang and C.-Y. Su, Chem. Sci., 2011, 2, 1396-1400.

216. A. Sahasrabudhe and S. Bhattacharyya, Chem. Mater., 2015, 27, 4848-4859.

217. Z. Ning, H. Tian, C. Yuan, Y. Fu, H. Qin, L. Sun and H. Agren, Chem. Commun., 2011, 47, 15361538.

218. P. K. Santra and P. V. Kamat, J. Am. Chem. Soc., 2013, 135, 877-885.

219. G. Wang, H. Wei, J. Shi, Y. Xu, H. Wu, Y. Luo, D. Li and Q. Meng, Nano Energy, 2017, 35, 1725.

220. L. Yue, H. Rao, J. Du, Z. Pan, J. Yu and X. Zhong, RSC Adv., 2018, 8, 3637-3645.

221. J.-Y. Kim, J. Yang, J. H. Yu, W. Baek, C.-H. Lee, H. J. Son, T. Hyeon and M. J. Ko, ACS Nano, 2015, 9, 11286-11295.

222. L. Zhang, Z. Pan, W. Wang, J. Du, Z. Ren, Q. Shen and X. Zhong, J. Mater. Chem. A, 2017, 5, 21442-21451.

223. W. Peng, J. Du, Z. Pan, N. Nakazawa, J. Sun, Z. Du, G. Shen, J. Yu, J. S. Hu, Q. Shen and X. Zhong, ACS Appl. Mater. Interfaces, 2017, 9, 5328-5336.

224. B. Bai, D. Kou, W. Zhou, Z. Zhou and S. Wu, Green Chem., 2015, 17, 4377-4382.

225.X. Michalet, F. F. Pinaud, L. A. Bentolila, J. M. Tsay, S. Doose, J. J. Li, G. Sundaresan, A. M. Wu,

S. S. Gambhir and S. Weiss, Science, 2005, 307, 538-544.

226. U. Resch-Genger, M. Grabolle, S. Cavaliere-Jaricot, R. Nitschke and T. Nann, Nat. Methods, 2008, 5, 763-775.

227. P. Zrazhevskiy, M. Sena and X. H. Gao, Chem. Soc. Rev., 2010, 39, 4326-4354.

228. N. Guijarro, T. Lana-Villarreal, I. Mora-Sero, J. Bisquert and R. Gomez, J. Phys. Chem. C, 2009, 113, 4208-4214.

229. S. Gimenez, I. Mora-Sero, L. Macor, N. Guijarro, T. Lana-Villarreal, R. Gomez, L. J. Diguna, Q.

Shen, T. Toyoda and J. Bisquert, Nanotechnology, 2009, 20, 295204.

230. H. J. Lee, J.-H. Yum, H. C. Leventis, S. M. Zakeeruddin, S. A. Haque, P. Chen, S. I. Seok, M.

Graetzel and M. K. Nazeeruddin, J. Phys. Chem. C, 2008, 112, 11600-11608.

231. W. Wang, G. Jiang, J. Yu, W. Wang, Z. Pan, N. Nakazawa, Q. Shen and X. Zhong, ACS Appl.

Mater. Interfaces, 2017, 9, 22549-22559.

232. A. Salant, M. Shalom, I. Hod, A. Faust, A. Zaban and U. Banin, ACS Nano, 2010, 4, 5962-5968. 233. X.-Y. Yu, J.-Y. Liao, K.-Q. Qiu, D.-B. Kuang and C.-Y. Su, ACS Nano, 2011, 5, 9494-9500. 
234. J. B. Sambur and B. A. Parkinson, J. Am. Chem. Soc., 2010, 132, 2130-2131.

235. I. Mora-Sero, S. Gimenez, T. Moehl, F. Fabregat-Santiago, T. Lana-Villareal, R. Gomez and J. Bisquert, Nanotechnology, 2008, 19, 424007.

236. V. L. Colvin, A. N. Goldstein and A. P. Alivisatos, J. Am. Chem. Soc., 1992, 114, 5221-5230.

237. I. Robel, V. Subramanian, M. Kuno and P. V. Kamat, J. Am. Chem. Soc., 2006, 128, 2385-2393.

238. B.-R. Hyun, Y.-W. Zhong, A. C. Bartnik, L. Sun, H. D. Abruna, F. W. Wise, J. D. Goodreau, J. R.

Matthews, T. M. Leslie and N. F. Borrelli, ACS Nano, 2008, 2, 2206-2212.

239. M.-H. Jung and M. G. Kang, J. Mater. Chem., 2011, 21, 2694-2700.

240. W. Lee, S. H. Kang, S. K. Min, Y.-E. Sung and S.-H. Han, Electrochem. Commun., 2008, 10, $1579-1582$.

241. S. Peng, F. Cheng, J. Liang, Z. Tao and J. Chen, J. Alloy. Comp., 2009, 481, 786-791.

242. X. Hu, Q. Zhang, X. Huang, D. Li, Y. Luo and Q. Meng, J. Mater. Chem., 2011, 21, 1590315905.

243. K. M. Coughlin, J. S. Nevins and D. F. Watson, ACS Appl. Mater. Interfaces, 2013, 5, 86498654.

244. J. S. Nevins, K. M. Coughlin and D. F. Watson, ACS Appl. Mater. Interfaces, 2011, 3, 42424253.

245. H. Wang, C. Luan, X. Xu, S. V. Kershaw and A. L. Rogach, J. Phys. Chem. C, 2012, 116, 484 489.

246. J. Luo, H. Wei, Q. Huang, X. Hu, H. Zhao, R. Yu, D. Li, Y. Luo and Q. Meng, Chem. Commun., 2013, 49, 3881-3883.

247. J. Luo, H. Wei, F. Li, Q. Huang, D. Li, Y. Luo and Q. Meng, Chem. Commun., 2014, 50, 3464 3466.

248. J. Y. Chang, C. H. Li, Y. H. Chiang, C. H. Chen and P. N. Li, ACS Appl. Mater. Interfaces, 2016, 8, 18878-18890.

249.Y.-H. Chiang, K.-Y. Lin, Y.-H. Chen, K. Waki, M. A. Abate, J.-C. Jiang and J.-Y. Chang, J. Mater. Chem. A, 2018, 6, 9629-9641.

250. A. Raevskaya, O. Rosovik, A. Kozytskiy, O. Stroyuk, V. Dzhagan and D. R. T. Zahn, RSC Adv, 2016, 6, 100145-100157.

251. H. Lee, H. C. Leventis, S.-J. Moon, P. Chen, S. Ito, S. A. Haque, T. Torres, F. Nüesch, T. Geiger,

S. M. Zakeeruddin, M. Grätzel and M. K. Nazeeruddin, Adv. Funct. Mater., 2009, 19, 2735-2742.

252. W.-T. Sun, Y. Yu, H.-Y. Pan, X.-F. Gao, Q. Chen and L.-M. Peng, J. Am. Chem. Soc., 2008, 130,

$1124-1125$.

253. J. H. Bang and P. V. Kamat, ACS Nano, 2009, 3, 1467-1476.

254. L. C. Spangler, L. Lu, C. J. Kiely, B. W. Berger and S. McIntosh, J. Mater. Chem. A, 2016, 4, 6107-6115.

255. G. Niu, L. Wang, R. Gao, B. Ma, H. Dong and Y. Qiu, J. Mater. Chem., 2012, 22, 16914-16919.

256. T. P. Brennan, O. Trejo, K. E. Roelofs, J. Xu, F. B. Prinz and S. F. Bent, J. Mater. Chem. A, 2013, 1, 7566-7571.

257. H. J. Lee, P. Chen, S.-J. Moon, F. Sauvage, K. Sivula, T. Bessho, D. R. Gamelin, P. Comte, S. M.

Zakeeruddin, S. I. Seok, M. Graetzel and M. K. Nazeeruddin, Langmuir, 2009, 25, 7602-7608.

258. J. Tian, T. Shen, X. Liu, C. Fei, L. Lv and G. Cao, Sci. Rep., 2016, 6, 23094.

259.J.-H. Yum, J.-W. Lee, Y. Kim, R. Humphry-Baker, N.-G. Park and M. Graetzel, Sol. Energy, 2014,

109, 183-188. 
260. B. Liu, D. Wang, Y. Zhang, H. Fan, Y. Lin, T. Jiang and T. Xie, Dalton Trans., 2013, 42, 22322237.

261. D. Wu, F. Wang, H. Wang, K. Cao, Z. Gao, F. Xua and K. Jiang, Dalton Trans., 2016, 45, 1627516282.

262. A. Tubtimtae, K.-L. Wu, H.-Y. Tung, M.-W. Lee and G. J. Wang, Electrochem. Commun., 2010, 12, 1158-1160.

263. M. A. Hines and P. Guyot-Sionnest, J. Phys. Chem., 1996, 100, 468-470.

264. X. G. Peng, M. C. Schlamp, A. V. Kadavanich and A. P. Alivisatos, J. Am. Chem. Soc., 1997, 119, 7019-7029.

265. S. L. Cumberland, K. M. Hanif, A. Javier, G. A. Khitrov, G. F. Strouse, S. M. Woessner and C. S. Yun, Chem. Mater., 2002, 14, 1576-1584.

266. L. Manna, E. C. Scher, L. S. Li and A. P. Alivisatos, J. Am. Chem. Soc., 2002, 124, 7136-7145.

267. J. J. Li, Y. A. Wang, W. Z. Guo, J. C. Keay, T. D. Mishima, M. B. Johnson and X. G. Peng, J. Am. Chem. Soc., 2003, 125, 12567-12575.

268. W. Zhang, G. Chen, J. Wang, B.-C. Ye and X. Zhong, Inorg. Chem., 2009, 48, 9723-9731.

269. W. Zhang, H. Zhang, Y. Feng and X. Zhong, ACS Nano, 2012, 6, 11066-11073.

270. P. Reiss, M. Protiere and L. Li, Small, 2009, 5, 154-168.

271. R. G. Chaudhuri and S. Paria, Chem. Rev., 2012, 112, 2373-2433.

272. D. Punnoose, C. S. S. P. Kumar, H. W. Seo, M. Shiratani, A. E. Reddy, S. S. Rao, C. V.

Thulasi-Varma, S.-K. Kim, S.-H. Chung and H.-J. Kim, New J. Chem., 2016, 40, 3423-3431.

273. J. Yang, J. Wang, K. Zhao, T. Izuishi, Y. Li, Q. Shen and X. Zhong, J. Phys. Chem. C, 2015, 119, 28800-28808.

274. M. D. Regulacio and M.-Y. Han, Acc. Chem. Res., 2010, 43, 621-630.

275. R. E. Bailey and S. M. Nie, J. Am. Chem. Soc., 2003, 125, 7100-7106.

276. X. H. Zhong, Y. Y. Feng, W. Knoll and M. Y. Han, J. Am. Chem. Soc., 2003, 125, 13559-13563.

277. X. H. Zhong, M. Y. Han, Z. L. Dong, T. J. White and W. Knoll, J. Am. Chem. Soc., 2003, 125, 8589-8594.

278. A. Kumar, K.-t. Li, A. R. Madaria and C. Zhou, Nano Res., 2011, 4, 1181-1190.

279. M. A. Hossain, J. R. Jennings, N. Mathews and Q. Wang, Phys. Chem. Chem. Phys., 2012, 14, 7154-7161.

280. M. G. Panthani, V. Akhavan, B. Goodfellow, J. P. Schmidtke, L. Dunn, A. Dodabalapur, P. F.

Barbara and B. A. Korgel, J. Am. Chem. Soc., 2008, 130, 16770-16777.

281. A. Chirila, P. Reinhard, F. Pianezzi, P. Bloesch, A. R. Uhl, C. Fella, L. Kranz, D. Keller, C.

Gretener, H. Hagendorfer, D. Jaeger, R. Erni, S. Nishiwaki, S. Buecheler and A. N. Tiwari, Nat. Mater., 2013, 12, 1107-1111.

282. I. Repins, M. A. Contreras, B. Egaas, C. DeHart, J. Scharf, C. L. Perkins, B. To and R. Noufi,

Prog. Photovoltaics, 2008, 16, 235-239.

283. C.-C. Chang, J.-K. Chen, C.-P. Chen, C.-H. Yang and J.-Y. Chang, ACS Appl. Mater. Interfaces, 2013, 5, 11296-11306.

284. J.-Y. Chang, J.-M. Lin, L.-F. Su and C.-F. Chang, ACS Appl. Mater. Interfaces, 2013, 5, 87408752.

285.Y.-q. Wang, Y.-c. Rui, Q.-h. Zhang, Y.-g. Li and H.-z. Wang, ACS Appl. Mater. Interfaces, 2013, 5, $11858-11864$.

286. J.-Y. Chang, L.-F. Su, C.-H. Li, C.-C. Chang and J.-M. Lin, Chem. Commun., 2012, 48, 4848- 
4850.

287. T.-L. Li, Y.-L. Lee and H. Teng, Energy Environ. Sci., 2012, 5, 5315-5324.

288. K.-T. Kuo, D.-M. Liu, S.-Y. Chen and C.-C. Lin, J. Mater. Chem., 2009, 19, 6780-6788.

289. T.-L. Li, Y.-L. Lee and H. Teng, J. Mater. Chem., 2011, 21, 5089-5098.

290. G. Xu, S. Ji, C. Miao, G. Liu and C. Ye, J. Mater. Chem., 2012, 22, 4890-4896.

291. N. S. Makarov, H. McDaniel, N. Fuke, I. Robel and V. I. Klimov, J. Phys. Chem. Lett., 2014, 5, 111-118.

292. J. Yang, J.-Y. Kim, J. H. Yu, T.-Y. Ahn, H. Lee, T.-S. Choi, Y.-W. Kim, J. Joo, M. J. Ko and T. Hyeon, Phys. Chem. Chem. Phys., 2013, 15, 20517-20525.

293.S. Higashimoto, M. Murano, T. Arase, S. Mukai, M. Azuma and M. Takahashi, Sol. Energy Mater.

Sol. Cells, 2017, 169, 203-209.

294. D. Aldakov, A. Lefrancois and P. Reiss, J. Mater. Chem. C, 2013, 1, 3756-3776.

295. M. G. Panthani, C. J. Stolle, D. K. Reid, D. J. Rhee, T. B. Harvey, V. A. Akhavan, Y. Yu and B. A.

Korgel, J. Phys. Chem. Lett., 2013, 4, 2030-2034.

296. D. Mocatta, G. Cohen, J. Schattner, O. Millo, E. Rabani and U. Banin, Science, 2011, 332, 7781.

297. N. Pradhan, D. Goorskey, J. Thessing and X. G. Peng, J. Am. Chem. Soc., 2005, 127, 1758617587.

298. N. Pradhan, D. M. Battaglia, Y. Liu and X. Peng, Nano Lett., 2007, 7, 312-317.

299. W. Zhang, Q. Lou, W. Ji, J. Zhao and X. Zhong, Chem. Mater, 2014, 26, 1204-1212.

300. W. Zhang, Y. Li, H. Zhang, X. Zhou and X. Zhong, Inorg. Chem., 2011, 50, 10432-10438.

301. W. Zhang, X. Zhou and X. Zhong, Inorg. Chem., 2012, 51, 3579-3587.

302. G. Rimal, A. K. Pimachev, A. J. Yost, U. Poudyal, S. Maloney, W. Wang, T. Chien, Y. Dahnovsky and J. Tang, Appl. Phys. Lett., 2016, 109, 103901.

303. M. P. A. Muthalif, Y.-S. Lee, C. D. Sunesh, H.-J. Kim and Y. Choe, Appl. Surf. Sci., 2017, 396, $582-589$.

304. L. Yu, Z. Li, Y. Liu, F. Cheng and S. Sun, Appl. Surf. Sci., 2014, 305, 359-365.

305. C. V. V. M. Gopi, M. Venkata-Haritha, S.-K. Kim and H.-J. Kim, Dalton Trans., 2015, 44, 630 638.

306. Z. Li, L. Yu, Y. Liu and S. Sun, Electrochim. Acta, 2015, 153, 200-209.

307. P. K. Santra and Y.-S. Chen, Electrochim. Acta, 2014, 146, 654-658.

308. S.-K. Kim, C. V. V. M. Gopi, J.-C. Lee and H.-J. Kim, J. Appl. Phys., 2015, 117, 163104.

309. N. Firoozi, H. Dehghani and M. Afrooz, J. Power Sources, 2015, 278, 98-103.

310. U. Poudyal, F. S. Maloney, K. Sapkota and W. Wang, Nanotechnology, 2017, 28, 415401.

311. I. Mehmood, Y. Liu, K. Chen, A. H. Shah and W. Chen, RSC Adv., 2017, 7, 33106-33112.

312. J. Wang, Y. Li, Q. Shen, T. Izuishi, Z. Pan, K. Zhao and X. Zhong, J. Mater. Chem. A, 2016, 4, 877-886.

313. M. A. Abbas, T.-Y. Kim, S. U. Lee, Y. S. Kang and J. H. Bang, J. Am. Chem. Soc., 2016, 138, 390-401.

314. Q. Tang, W. Zhu, B. He and P. Yang, ACS Nano, 2017, 11, 1540-1547.

315. J. Briscoe, A. Marinovic, M. Sevilla, S. Dunn and M. Titirici, Angew. Chem. Int. Ed., 2015, 54, $4463-4468$.

316. K.-H. Ye, Z. Wang, J. Gu, S. Xiao, Y. Yuan, Y. Zhu, Y. Zhang, W. Mai and S. Yang, Energy Environ. Sci., 2017, 10, 772-779. 
317. P. Mirtchev, E. J. Henderson, N. Soheilnia, C. M. Yip and G. A. Ozin, J. Mater. Chem., 2012, 22, $1265-1269$.

318. X. Yan, X. Cui, B. Li and L. S. Li, Nano Lett., 2010, 10, 1869-1873.

319. H. Wang, P. Sun, S. Cong, J. Wu, L. Gao, Y. Wang, X. Dai, Q. Yi and G. Zou, Nanoscale Res. Lett., 2016, 11, 27.

320. V. M. Blas-Ferrando, J. Ortiz, V. Gonzalez-Pedro, R. S. Sanchez, I. Mora-Sero, F. Fernandez-Lazaro and A. Sastre-Santos, Adv. Funct. Mater., 2015, 25, 3220-3226.

321. J.-G. Song, X. Song, T. Ling, X.-W. Du and S. Z. Qiao, Ind. Eng. Chem. Res., 2012, 51, 1007410078 .

322. S. Sarkar, A. Makhal, K. Lakshman, T. Bora, J. Dutta and S. K. Pal, J. Phys. Chem., 2012, 116, $14248-14256$.

323. J. Chen, W. Lei and W. Q. Deng, Nanoscale, 2011, 3, 674-677.

324. Y. Liu and J. Wang, Thin Solid Films, 2010, 518, E54-E56.

325. H. Choi, R. Nicolaescu, S. Paek, J. Ko and P. V. Kamat, ACS Nano, 2011, 5, 9238-9245.

326.S. Buhbut, S. Itzhakov, E. Tauber, M. Shalom, I. Hod, T. Geiger, Y. Garini, D. Oron and A. Zaban, ACS Nano, 2010, 4, 1293-1298.

327. M. Shalom, J. Albero, Z. Tachan, E. Martinez-Ferrero, A. Zaban and E. Palomares, J. Phys. Chem. Lett., 2010, 1, 1134-1138.

328. J. Dana, P. Anand, S. Maiti, F. Azlan, Y. Jadhav, S. K. Haram and H. N. Ghosh, J. Phys. Chem. C, 2017, 122, 13277-13284.

329. D. Van-Duong, P. Kim, S. Baek, L. L. Larina, K. Yong, R. Ryoo, S. H. Ko and H.-S. Choi, Carbon, 2016, 96, 139-144.

330. G. Zhu, L. Pan, T. Xu and Z. Sun, ACS Appl. Mater. Interfaces, 2011, 3, 3146-3151.

331. G. Zhu, L. Pan, T. Xu and Z. Sun, ACS Appl. Mater. Interfaces, 2011, 3, 1472-1478.

332. G. Zhu, L. Pan, H. Sun, X. Liu, T. Lv, T. Lu, J. Yang and Z. Sun, ChemPhysChem, 2012, 13, 769-773.

333. G. Hodes, J. Manassen and D. Cahen, J. Electrochem. Soc., 1980, 127, 544-549.

334. A. D. Savariraj, K. K. Viswanathan and K. Prabakar, ACS Appl. Mater. Interfaces, 2014, 6, 19702-19709.

335. Y. Jiang, X. Zhang, Q.-Q. Ge, B.-B. Yu, Y.-G. Zou, W.-J. Jiang, J.-S. Hu, W.-G. Song and L.-J. Wan, ACS Appl. Mater. Interfaces, 2014, 6, 15448-15455.

336. M. Ye, C. Chen, N. Zhang, X. Wen, W. Guo and C. Lin, Adv. Energy Mater, 2014, 4, 1301564.

337. X. Q. Chen, Z. Li, Y. Bai, Q. Sun, L. Z. Wang and S. X. Dou, Chem-Eur. J., 2015, 21, 10551063.

338. S. S. Kalanur, S. Y. Chae and O. S. Joo, Electrochim. Acta, 2013, 103, 91-95.

339. F. Xi, H. Liu, W. Li, L. Zhu, H. Geng, L. Quan and W. Liang, Electrochim. Acta, 2015, 178, 329335.

340. C. Shen, L. Sun, Z. Y. Koh and Q. Wang, J. Mater. Chem. A, 2014, 2, 2807-2813.

341. M. Ye, X. Wen, N. Zhang, W. Guo, X. Liu and C. Lin, J. Mater. Chem. A, 2015, 3, 9595-9600.

342. F. Wang, H. Dong, J. Pan, J. Li, Q. Li and D. Xu, J. Phys. Chem. C, 2014, 118, 19589-19598.

343. W. Ke, G. Fang, H. Lei, P. Qin, H. Tao, W. Zeng, J. Wang and X. Zhao, J. Power Sources, 2014, 248, 809-815.

344. Y. Wang, Q. Zhang, Y. Li and H. Wang, J. Power Sources., 2016, 318, 128-135.

345. G. S. Selopal, I. Concina, R. Milan, M. M. Natile, G. Sberveglieri and A. Vomiero, Nano Energy, 
2014, 6, 200-210.

346. Y. Jiang, B.-B. Yu, J. Liu, Z.-H. Li, J.-K. Sun, X.-H. Zhong, J.-S. Hu, W.-G. Song and L.-J. Wan,

Nano Lett., 2015, 15, 3088-3095.

347. Y. Jiang, X. Zhang, Q.-Q. Ge, B.-B. Yu, Y.-G. Zou, W.-J. Jiang, W.-G. Song, L.-J. Wan and J.-S. Hu, Nano Lett., 2014, 14, 365-372.

348.A. D. Savariraj, G. Rajendrakumar, S. Selvam, S. N. Karthick, B. Balamuralitharan, H.-J. Kim, K.

K. Viswanathan, M. Vijayakumar and K. Prabakar, RSC Adv., 2015, 5, 100560-100567.

349. K. Zhao, H. Yu, H. Zhang and X. Zhong, J. Phys. Chem. C, 2014, 118, 5683-5690.

350. H. Zhang, H. Bao and X. Zhong, J. Mater. Chem. A, 2015, 3, 6557-6564.

351. M. Deng, S. Huang, Q. Zhang, D. Li, Y. Luo, Q. Shen, T. Toyoda and Q. Meng, Chem. Lett., 2010, 39, 1168-1170.

352. H. M. Choi, I. A. Ji and J. H. Bang, ACS Appl. Mater. Interfaces, 2014, 6, 2335-2343.

353. F. Bo, C. Zhang, C. Wang, S. Xu, Z. Wang and Y. Cui, J. Mater. Chem. A, 2014, 2, 14585-14592.

354. S. Wang, T. Shen, H. Bai, B. Li and J. Tian, J. Mater. Chem. C, 2016, 4, 8020-8026.

355. M. Eskandari and V. Ahmadi, Mater. Lett., 2015, 142, 308-311.

356. Y. Bai, C. Han, X. Chen, H. Yu, X. Zong, Z. Li and L. Wang, Nano Energy, 2015, 13, 609-619.

357. H. Zhang, C. Wang, W. Peng, C. Yang and X. Zhong, Nano Energy, 2016, 23, 60-69.

358. B.-M. Kim, M.-K. Son, S.-K. Kim, N.-Y. Hong, S. Park, M.-S. Jeong, H. Seo, K. Prabakar and H.-J. Kim, Electrochim. Acta, 2014, 117, 92-98.

359. C.-Y. Lin, C.-Y. Teng, T.-L. Li, Y.-L. Lee and H. Teng, J. Mater. Chem. A, 2013, 1, 1155-1162.

360. C. V. Thulasi-Varma, S. S. Rao, K. D. Ikkurthi, S.-K. Kim, T.-S. Kang and H.-J. Kim, J. Mater. Chem. C, 2015, 3, 10195-10206.

361. C. V. V. M. Gopi, J.-H. Bae, M. Venkata-Haritha, S.-K. Kim, Y.-S. Lee, G. Sarat and H.-J. Kim, RSC Adv., 2015, 5, 107522-107532.

362. Z. Tachan, M. Shalom, I. Hod, S. Ruehle, S. Tirosh and A. Zaban, J. Phys. Chem. C, 2011, 115, 6162-6166.

363. K. Meng and K. R. Thampi, ACS Appl. Mater. Interfaces, 2014, 6, 20768-20775.

364. C. Chen, M. Ye, N. Zhang, X. Wen, D. Zheng and C. Lin, J. Mater. Chem. A, 2015, 3, 63116314.

365. M. S. Faber, K. Park, M. Caban-Acevedo, P. K. Santra and S. Jin, J. Phys. Chem. Lett., 2013, 4, $1843-1849$.

366. Q. Luo, Y. Gu, J. Li, N. Wang and H. Lin, J. Power Sources, 2016, 312, 93-100.

367. R. Seenu, C. V. V. M. Gopi, S. S. Rao, S.-K. Kim and H.-J. Kim, Electrochim. Acta, 2016, 204, $255-262$.

368. H. Chen, L. Zhu, H. Liu and W. Li, J. Power Sources, 2014, 245, 406-410.

369. L. Quan, W. Li, L. Zhu, H. Geng, X. Chang and H. Liu, J. Power Sources, 2014, 272, 546-553.

370. J. Xu, H. Xue, X. Yang, H. Wei, W. Li, Z. Li, W. Zhang and C.-S. Lee, Small, 2014, 10, 4754 4759.

371. H.-J. Kim, D.-J. Kim, S. S. Rao, A. D. Savariraj, K. Soo-Kyoung, M.-K. Son, C. V. V. M. Gopi and K. Prabakar, Electrochim. Acta, 2014, 127, 427-432.

372. C. V. V. M. Gopi, S. S. Rao, S.-K. Kim, D. Punnoose and H.-J. Kim, J. Power Sources., 2015, 275, 547-556.

373. Y. Zhang, C. Shi, X. Dai, F. Liu, X. Fang and J. Zhu, Electrochim. Acta, 2014, 118, 41-44.

374. D. Barpuzary, A. Banik, G. Gogoi and M. Qureshi, J. Mater. Chem. A, 2015, 3, 14378-14388. 
375. X. Dai, C. Shi, Y. Zhang, F. Liu, X. Fang and J. Zhu, Mater. Lett., 2014, 116, 251-253.

376. Y. Cao, Y. Xiao, J.-Y. Jung, H.-D. Um, S.-W. Jee, H. M. Choi, J. H. Bang and J.-H. Lee, ACS Appl. Mater. Interfaces, 2013, 5, 479-484.

377. X. Zeng, W. Zhang, Y. Xie, D. Xiong, W. Chen, X. Xu, M. Wang and Y.-B. Cheng, J. Power Sources, 2013, 226, 359-362.

378. X. Zeng, D. Xiong, W. Zhang, L. Ming, Z. Xu, Z. Huang, M. Wang, W. Chen and Y.-B. Cheng, Nanoscale, 2013, 5, 6992-6998.

379. J. Xu, X. Yang, Q.-D. Yang, T.-L. Wong and C.-S. Lee, J. Phys. Chem. C, 2012, 116, $19718-$ 19723.

380. X. Zeng, W. Zhang, Y. Xie, D. Xiong, W. Chen, X. Xu, M. Wang and Y.-B. Cheng, J. Power Sources, 2013, 226, 359-362.

381. A. Kay and M. Gratzel, Sol. Energy Mater. Sol. Cells, 1996, 44, 99-117.

382.G. S. Paul, J. H. Kim, M.-S. Kim, K. Do, J. Ko and J.-S. Yu, ACS Appl. Mater. Interfaces, 2012, 4, 375-381.

383. S.-Q. Fan, B. Fang, J. H. Kim, J.-J. Kim, J.-S. Yu and J. Ko, Appl. Phys. Lett., 2010, 96, 063501.

384. V.-D. Dao, Y. Choi, K. Yong, L. L. Larina and H.-S. Choi, Carbon, 2015, 84, 383-389.

385. P. Sudhagar, E. Ramasamy, W.-H. Cho, J. Lee and Y. S. Kang, Electrochem. Commun., 2011, 13, 34-37.

386. J. Dong, S. Jia, J. Chen, B. Li, J. Zheng, J. Zhao, Z. Wang and Z. Zhu, J. Mater. Chem., 2012, 22, 9745-9750.

387. C. V. V. M. Gopi, M. Venkata-Haritha, S.-K. Kim and H.-J. Kim, J. Power Sources, 2016, 311, $111-120$.

388. Q. Zhang, S. Zhou, Q. Li and H. Li, RSC Adv, 2015, 5, 30617-30623.

389. A. Sahasrabudhe, S. Kapri and S. Bhattacharyya, Carbon, 2016, 107, 395-404.

390. Q. Zhang, Y. Zhang, S. Huang, X. Huang, Y. Luo, Q. Meng and D. Li, Electrochem. Commun., 2010, 12, 327-330.

391. J. Yu, W. Wang, Z. Pan, J. Du, Z. Ren, W. Xue and X. Zhong, J. Mater. Chem. A, 2017, 5, 1412414133.

392. H. Zhang, C. Yang, Z. Du, D. Pan and X. Zhong, J. Mater. Chem. A, 2017, 5, 1614-1622.

393. H.-J. Kim, T.-B. Yeo, S.-K. Kim, S. S. Rao, A. D. Savariraj, K. Prabakar and C. V. V. M. Gopi, Eur. J. Inorg. Chem., 2014, 4281-4286.

394. D. Wu, Y. Wang, F. Wang, H. Wang, Y. An, Z. Gao, F. Xu and K. Jiang, Carbon, 2017, 123, 756766.

395. F. Liu, J. Zhu, Y. Li, J. Wei, M. Lv, Y. Xu, L. Zhou, L. Hu and S. Dai, J. Power Sources, 2015, 292, 7-14.

396. D.-M. Li, L.-Y. Cheng, Y.-D. Zhang, Q.-X. Zhang, X.-M. Huang, Y.-H. Luo and Q.-B. Meng, Sol. Energy Mater. Sol. Cells, 2014, 120, 454-461.

397. J. G. Radich, R. Dwyer and P. V. Kamat, J. Phys. Chem. Lett., 2011, 2, 2453-2460.

398. Y. Yang, L. Zhu, H. Sun, X. Huang, Y. Luo, D. Li and Q. Meng, ACS Appl. Mater. Interfaces, $2012,4,6162-6168$.

399. X. Zhang, X. Huang, Y. Yang, S. Wang, Y. Gong, Y. Luo, D. Li and Q. Meng, ACS Appl. Mater. Interfaces, 2013, 5, 5954-5960.

400. D. Ghosh, G. Halder, A. Sahasrabudhe and S. Bhattacharyya, Nanoscale, 2016, 8, 10632-10641. 401. P. Parand, M. Samadpour, A. Esfandiar and A. I. Zad, ACS Photonics, 2014, 1, 323-330. 
402. M. Eskandari, V. Ahmadi and R. Ghahary, Electrochim. Acta, 2015, 151, 393-398.

403. L. Givalou, M. Antoniadou, D. Perganti, M. Giannouri, C.-S. Karagianni, A. G. Kontos and P. Falaras, Electrochim. Acta, 2016, 210, 630-638.

404. M. Que, W. Guo, X. Zhang, X. Li, Q. Hua, L. Dong and C. Pan, J. Mater. Chem. A, 2014, 2, 13661-13666.

405. J. H. Zeng, D. Chen, Y. F. Wang and B. B. Jin, J. Mater. Chem. C, 2015, 3, 12140-12148.

406. L. Li, P. Zhu, S. Peng, M. Srinivasan, Q. Yan, A. S. Nair, B. Liu and S. Samakrishna, J. Phys. Chem. C, 2014, 118, 16526-16535.

407.H.-J. Kim, S.-W. Kim, C. V. V. M. Gopi, S.-K. Kim, S. S. Rao and M.-S. Jeong, J. Power Sources, 2014, 268, 163-170.

408. X. Song, M. Wang, J. Deng, Y. Ju, T. Xing, J. Ding, Z. Yang and J. Shao, J. Power Sources, 2014, 269, 661-670.

409. S. S. Khalili and H. Dehghani, RSC Adv., 2016, 6, 10880-10886.

410. G. Yue, F. Tan, J. Wu, F. Li, J. Lin, M. Huang and W. Zhang, RSC Adv., 2015, 5, 42101-42108.

411. C. V. V. M. Gopi, M. Venkata-Haritha, S. Ravi, C. V. Thulasi-Varma, S.-K. Kim and H.-J. Kim, J. Mater. Chem. C, 2015, 3, 12514-12528.

412. P. S. Tamboli, M. B. R. Prasad, V. S. Kadam, R. S. Vhatkar, Inamuddin, H. M. Pathan and S. S.

Mahajan, Sol. Energy Mater. Sol. Cells, 2017, 161, 96-101.

413. S. Licht, Sol. Energy Mater. Sol. Cells, 1995, 38, 305-319.

414. Y.-L. Lee and C.-H. Chang, J. Power Sources, 2008, 185, 584-588.

415. L. Li, X. Yang, J. Gao, H. Tian, J. Zhao, A. Hagfeldt and L. Sun, J. Am. Chem. Soc., 2011, 133, 8458-8460.

416. Z. Ning, C. Yuan, H. Tian, Y. Fu, L. Li, L. Sun and H. Ågren, J. Mater. Chem., 2012, 22, 60326037.

417. V. Jovanovski, V. Gonzalez-Pedro, S. Gimenez, E. Azaceta, G. Cabanero, H. Grande, R. Tena-Zaera, I. Mora-Sero and J. Bisquert, J. Am. Chem. Soc., 2011, 133, 20156-20159.

418. R. M. Evangelista, S. Makuta, S. Yonezu, J. Andrews and Y. Tachibana, ACS Appl. Mater. Interfaces, 2016, 8, 13957-13965.

419. S. Y. Chae, Y. J. Hwang and O.-S. Joo, RSC Adv., 2014, 4, 26907-26911.

420. R. Dang, Y. Wang, J. Zeng, Z. Huang, Z. Fei and P. J. Dyson, J. Mater. Chem. A, 2017, 5, 1352613534.

421. C. Levy-Clement, R. Tena-Zaera, M. A. Ryan, A. Katty and G. Hodes, Adv. Mater, 2005, 17, $1512-1515$.

422. J. P. Park, J. H. Heo, S. H. Im and S.-W. Kim, J. Mater. Chem. A, 2016, 4, 785-790.

423. S. H. Im, C. S. Lim, J. A. Chang, Y. H. Lee, N. Maiti, H. J. Kim, M. K. Nazeeruddin, M. Gratzel and S. I. Seok, Nano Lett., 2011, 11, 4789-4793.

424. J. Du, X. Meng, K. Zhao, Y. Li and X. Zhong, J. Mater. Chem. A, 2015, 3, 17091-17097.

425. H. Wei, G. Wang, J. Shi, H. Wu, Y. Luo, D. Li and Q. Meng, J. Mater. Chem. A, 2016, 4, 1419414203.

426. W. Feng, L. Zhao, J. Du, Y. Li and X. Zhong, J. Mater. Chem. A, 2016, 4, 14849-14856.

427.Z. Yu, Q. Zhang, D. Qin, Y. Luo, D. Li, Q. Shen, T. Toyoda and Q. Meng, Electrochem. Commun., 2010, 12, 1776-1779.

428. S. Wang, Q.-X. Zhang, Y.-Z. Xu, D.-M. Li, Y.-H. Luo and Q.-B. Meng, J. Power Sources, 2013, 224, 152-157. 
429. H.-Y. Chen, L. Lin, X.-Y. Yu, K.-Q. Qiu, X.-Y. Lu, D.-B. Kuang and C.-Y. Su, Electrochim. Acta, 2013, 92, 117-123.

430. J. Duan, Q. Tang, R. Li, B. He, L. Yu and P. Yang, J. Power Sources, 2015, 284, 369-376.

431. Z. Huo, L. Tao, S. Wang, J. Wei, J. Zhu, W. Dong, F. Liu, S. Chen, B. Zhang and S. Dai, J. Power Sources, 2015, 284, 582-587.

432. W. Feng, Y. Li, J. Du, W. Wang and X. Zhong, J. Mater. Chem. A, 2016, 4, 1461-1468.

433. G. Hodes and D. Cahen, Acc. Chem. Res., 2012, 45, 705-713.

434. S. H. Im, H.-j. Kim, S. W. Kim, S.-W. Kim and S. I. Seok, Energy Environ. Sci., 2011, 4, 41814186.

435. D. C. Neo, N. Zhang, Y. Tazawa, H. Jiang, G. M. Hughes, C. R. Grovenor, H. E. Assender and A.

A. Watt, ACS Appl. Mater. Interfaces, 2016, 8, 12101-12108.

436. G. Larramona, C. Chone, A. Jacob, D. Sakakura, B. Delatouche, D. Pere, X. Cieren, M. Nagino and R. Bayon, Chem. Mater, 2006, 18, 1688-1696.

437. C.-F. Chi, P. Chen, Y.-L. Lee, I. P. Liu, S.-C. Chou, X.-L. Zhang and U. Bach, J. Mater. Chem., 2011, 21, 17534-17540.

438. J. Qian, Q. S. Liu, G. Li, K. J. Jiang, L. M. Yang and Y. Song, Chem. Commun., 2011, 47, 64616463.

439. G. Sfyri, S. Sfaelou, K. S. Andrikopoulos, N. Balis, G. A. Voyiatzis and P. Lianos, J. Phys. Chem. C, 2014, 118, 16547-16551.

440. Y. H. Lee, S. H. Im, J. A. Chang, J.-H. Lee and S. I. Seok, Org. Electron., 2012, 13, 975-979.

441. Y. Itzhaik, O. Niitsoo, M. Page and G. Hodes, J. Phys. Chem. C, 2009, 113, 4254-4256.

442. J. A. Chang, J. H. Rhee, S. H. Im, Y. H. Lee, H. J. Kim, S. I. Seok, M. K. Nazeeruddin and M. Gratzel, Nano Lett., 2010, 10, 2609-2612.

443. S.-J. Moon, Y. Itzhaik, J.-H. Yum, S. M. Zakeeruddin, G. Hodes and M. Graetzel, J. Phys. Chem. Lett., 2010, 1, 1524-1527.

444. S. Nezu, G. Larramona, C. Chone, A. Jacob, B. Delatouche, D. Pere and C. Moisan, J. Phys. Chem. C, 2010, 114, 6854-6859.

445.J. A. Chang, S. H. Im, Y. H. Lee, H. J. Kim, C. S. Lim, J. H. Heo and S. I. Seok, Nano Lett., 2012,

12, 1863-1867.

446. C. S. Lim, S. H. Im, J. A. Chang, Y. H. Lee, H. J. Kim and S. I. Seok, Nanoscale, 2012, 4, 429432.

447. C. S. Lim, S. H. Im, H. J. Kim, J. A. Chang, Y. H. Lee and S. I. Seok, Phys. Chem. Chem. Phys., 2012, 14, 3622-3626.

448. C.-S. Lim, S. H. Im, J. H. Rhee, Y. H. Lee, H.-J. Kim, N. Maiti, Y. Kang, J. A. Chang, M. K.

Nazeeruddin, M. Grätzel and S. I. Seok, J. Mater. Chem., 2012, 22, 1107-1111.

449. P. P. Boix, Y. H. Lee, F. Fabregat-Santiago, S. H. Im, I. Mora-Sero, J. Bisquert and S. I. Seok, ACS Nano, 2012, 6, 873-880.

450. Y. C. Choi, Y. H. Lee, S. H. Im, J. H. Noh, T. N. Mandal, W. S. Yang and S. I. Seok, Adv. Energy Mater., 2014, 4, 1301680.

451. G. M. Peng, X. Q. Xu, F. J. Mei, G. Xu, J. M. Wu, D. Gao, J. E. Ellis, Y. Zhao, Y. Xing and A. Star, RSC Adv., 2014, 4, 53335-53343.

452. Y. C. Choi and S. I. Seok, Adv. Funct. Mater, 2015, 25, 2892-2898.

453. Y. C. Choi, E. J. Yeom, T. K. Ahn and S. I. Seok, Angew. Chem. Int. Ed., 2015, 54, 4005-4009.

454. K. C. Godel, Y. C. Choi, B. Roose, A. Sadhanala, H. J. Snaith, S. I. Seok, U. Steiner and S. K. 
Pathak, Chem. Commun., 2015, 51, 8640-8643.

455. K. Tsujimoto, D.-C. Nguyen, S. Ito, H. Nishino, H. Matsuyoshi, A. Konno, G. R. A. Kumara and

K. Tennakone, J. Phys. Chem. C, 2012, 116, 13465-13471.

456. Y. C. Choi, T. N. Mandal, W. S. Yang, Y. H. Lee, S. H. Im, J. H. Noh and S. I. Seok, Angew. Chem. Int. Ed., 2014, 53, 1329-1333.

457. H.-Y. Wei, G.-S. Wang, H.-J. Wu, Y.-H. Luo, D.-M. Li and Q.-B. Meng, Acta Phys-Chim. Sin., 2016, 32, 201-213.

458. K. E. Roelofs, T. P. Brennan, J. C. Dominguez, C. D. Bailie, G. Y. Margulis, E. T. Hoke, M. D.

McGehee and S. F. Bent, J. Phys. Chem. C, 2013, 117, 5584-5592.

459. Q. Shen, J. Kobayashi, L. J. Diguna and T. Toyoda, J. Alloy. Phys., 2008, 103, 084304.

460. S. Yang, C. Huang, J. Zhai, Z. Wang and L. Jiang, J. Mater. Chem., 2002, 12, 1459-1464.

461. S.-H. Hsu, S.-F. Hung and S.-H. Chien, J. Power Sources, 2013, 233, 236-243.

462. Y.-S. Lee, C. V. V. M. Gopi, A. E. Reddy, C. Nagaraju and H.-J. Kim, New J. Chem., 2017, 41, 1914-1917.

463. S. S. Rao, I. K. Durga, C. V. Tulasi-Varma, D. Punnoose, S.-K. Kim and H.-J. Kim, Org. Electron., 2015, 26, 200-207.

464. N. Guijarro, J. M. Campina, Q. Shen, T. Toyoda, T. Lana-Villarreal and R. Gomez, Phys. Chem.

Chem. Phys., 2011, 13, 12024-12032.

465. Z. Peng, Y. Liu, Y. Zhao, K. Chen, Y. Cheng, V. Kovalev and W. Chen, J. Alloy. Comp., 2014, 587, 613-617.

466. F. Huang, Q. Zhang, B. Xu, J. Hou, Y. Wang, R. C. Masse, S. Peng, J. Liu and G. Cao, J. Mater. Chem. A, 2016, 4, 14773-14780.

467. F. Huang, J. Hou, H. Wang, H. Tang, Z. Liu, L. Zhang, Q. Zhang, S. Peng, J. Liu and G. Cao, Nano Energy, 2017, 32, 433-440.

468. F. Huang, J. Hou, Q. Zhang, Y. Wang, R. C. Masse, S. Peng, H. Wang, J. Liu and G. Cao, Nano

Energy, 2016, 26, 114-122.

469. A. Subramanian, D. Punnoose, S. S. Rao, C. V. T. Varma, B. Naresh, V. Raman and H.-J. Kim, New J. Chem., 2017, 41, 5942-5949.

470. Z. Liu, M. Miyauchi, Y. Uemura, Y. Cui, K. Hara, Z. Zhao, K. Sunahara and A. Furube, Appl.

Phys. Lett., 2010, 96, 233107.

471. M. Shalom, S. Dor, S. Ruhle, L. Grinis and A. Zaban, J. Phys. Chem. C, 2009, 113, 3895-3898.

472. Z. Tachan, I. Hod, M. Shalom, L. Grinis and A. Zaban, Phys. Chem. Chem. Phys., 2013, 15, 3841-3845.

473. Z. Pan and X. Zhong, J. Mater. Chem. A, 2016, 4, 18976-18982.

474. A. K. Chandiran, M. K. Nazeeruddin and M. Graetzel, Adv. Funct. Mater., 2014, 24, 1615-1623.

475. A. Kay and M. Gratzel, Chem. Mater., 2002, 14, 2930-2935.

476. E. Palomares, J. N. Clifford, S. A. Haque, T. Lutz and J. R. Durrant, J. Am. Chem. Soc., 2003, 125, 475-482.

477. J. Guo, C. She and T. Lian, J. Phys. Chem. C, 2007, 111, 8979-8987.

478. T. Shen, L. Bian, B. Li, K. Zheng, T. Pullerits and J. Tian, Appl. Phys. Lett., 2016, 108, 213901.

479. S. Maiti, F. Azlan, P. Anand, Y. Jadhav, J. Dana, S. K. Haram and H. N. Ghosh, Langmuir, 2018, 34, 50-57.

480. E. M. Barea, M. Shalom, S. Gimenez, I. Hod, I. Mora-Sero, A. Zaban and J. Bisquert, J. Am. Chem. Soc., 2010, 132, 6834-6839. 
481. M. Solis de la Fuente, R. S. Sanchez, V. Gonzalez-Pedro, P. P. Boix, S. G. Mhaisalkar, M. E. Rincon, J. Bisquert and I. Mora-Sero, J. Phys. Chem. Lett., 2013, 4, 1519-1525.

482.A. N. Jumabekov, N. Cordes, T. D. Siegler, P. Docampo, A. Ivanova, K. Fominykh, D. D. Medina,

L. M. Peter and T. Bein, ACS Appl. Mater. Interfaces, 2016, 8, 4600-4607.

483. P. N. Kumar, A. Kolay, M. Deepa, S. M. Shivaprasad and A. K. Srivastava, ACS Appl. Mater. Interfaces, 2017, 9, 25278-25290.

484. H. I. Wang, H. Lu, Y. Nagata, M. Bonn and E. Canovas, ACS Nano, 2017, 11, 4760-4767.

485. X. Zhao, R. N. Ma, M. X. Yang, H. D. Yang, P. Jin, Z. Q. Li, Y. Z. Fan, A. Du and X. M. Cao, J. Alloy. Comp., 2017, 726, 593-600.

486. P. Cheng and X. Zhan, Chem. Soc. Rev., 2016, 45, 2544-2582.

487. K. Domanski, E. A. Alharbi, A. Hagfeldt, M. Grätzel and W. Tress, Nat. Energy, 2018, 3, 61-67.

488. M. Saliba, Science, 2018, 359, 388-389.

489. N. Arora, M. I. Dar, A. Hinderhofer, N. Pellet, F. Schreiber, S. M. Zakeeruddin and M. Graetzel, Science, 2017, 358, $768-771$.

490. W. Chen, Y. Wu, Y. Yue, J. Liu, W. Zhang, X. Yang, H. Chen, E. Bi, I. Ashraful, M. Graetzel and

L. Han, Science, 2015, 350, 944-948.

491.H. Tan, A. Jain, O. Voznyy, X. Lan, F. P. G. de Arquer, J. Z. Fan, R. Quintero-Bermudez, M. Yuan,

B. Zhang, Y. Zhao, F. Fan, P. Li, L. N. Quan, Y. Zhao, Z.-H. Lu, Z. Yang, S. Hoogland and E. H.

Sargent, Science, 2017, 355, 722-726.

492. C. V. V. M. Gopi, M. Venkata-Haritha, S.-K. Kim and H.-J. Kim, Nanoscale, 2015, 7, $12552-$ 12563.

493. S. Sfaelou, A. G. Kontos, L. Givalou, P. Falaras and P. Lianos, Catal. Today, 2014, 230, 221-226.

494. Y. Chen, Q. Tao, W. Fu, H. Yang, X. Zhou, Y. Zhang, S. Su, P. Wang and M. Li, Electrochim. Acta, 2014, 118, 176-181.

495. W. Guo, Z. Du, Q. Zhao, H. Zhang and X. Zhong, J. Phys. Chem. C, 2016, 120, 16500-16506.

496. S. Wang, Q.-X. Zhang, Y.-Z. Xu, D.-M. Li, Y.-H. Luo and Q.-B. Meng, J. Power Sources, 2013, 224, 152-157.

497. H. Kim, I. Hwang and K. Yong, ACS Appl. Mater. Interfaces, 2014, 6, 11245-11253.

498.A. Fakharuddin, R. Jose, T.M. Brown, F. Fabregat-Santiago and J. Bisquert, Energy Environ. Sci., 2014,7, 3952-3981. 


\section{Table of contents (TOC)}

Quantum dot-sensitized solar cells: a promising candidate for the next-generation solar cells

Zhenxiao Pan, Huashang Rao, Iván Mora-Seró, Juan Bisquert and Xinhua Zhong

A comprehensive overview of the development of quantum dot-sensitized solar cells (QDSCs) is presented.

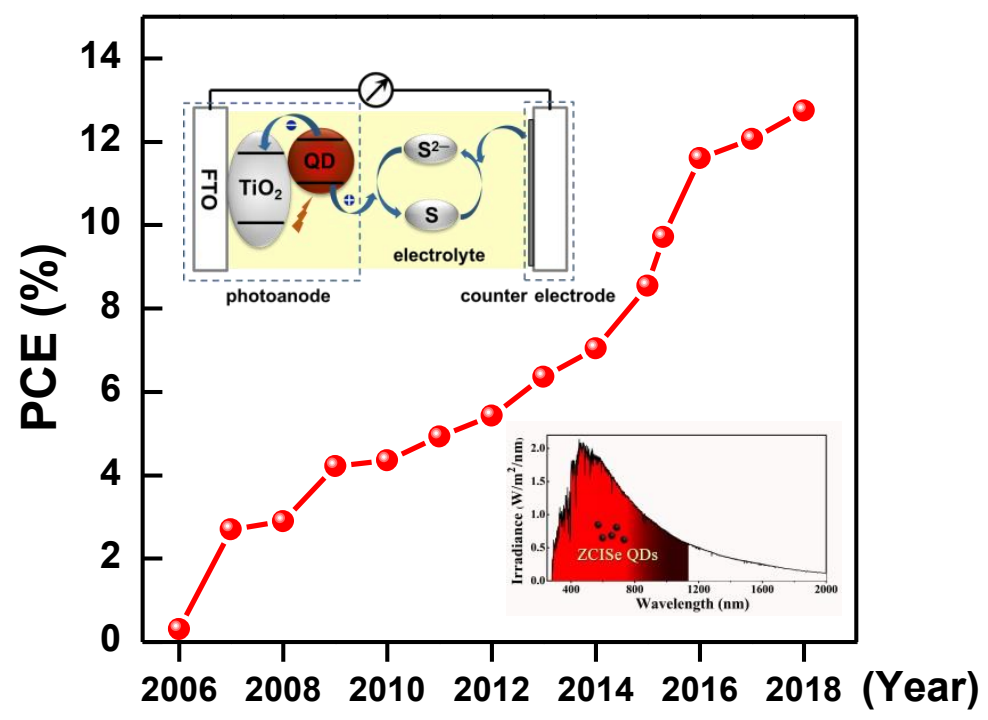

